\author{
Aus der Abteilung \\ Gastroenterologie und Endokrinologie \\ (Prof. Dr. med. Dr. h.c. G. Ramadori) \\ im Zentrum Innere Medizin
}

der Medizinischen Fakultät der Universität Göttingen

\title{
Die perioperative Chemotherapie des lokal fortgeschrittenen Magenkarzinoms nach
}

\section{Göttinger Erfahrung}

\author{
INAUGURAL-DISSERTATION \\ zur Erlangung des Doktorgrades \\ für Zahnheilkunde \\ der Medizinischen Fakultät der \\ Georg-August-Universität zu Göttingen
}

vorgelegt von

Dierck-Henrik Röper

aus

Münster / Westfalen

Göttingen, 2011 
Dekan:

I. Berichterstatter:

II. Berichterstatter/in:

III. Berichterstatter/in:

Tag der mündlichen Prüfung: $\quad$ 20.06.2012
Prof. Dr. med. C. Frömmel

Prof. Dr. med. Dr. h.c. G. Ramadori

Priv.- Doz. Dr. med. T. Liersch

Prof. Dr. med. dent. R. Mausberg 
$\begin{array}{lll}\text { 1. } & \text { Einleitung und Fragestellung } & 7\end{array}$

$\begin{array}{lll}2 . & \text { Pathologie des Magenkarzinoms } & 9\end{array}$

2.1 Das Magenkarzinom als neoplastische Läsion der Magenschleimhaut

2.1.1 Magenfrühkarzinom

2.1 .2 Fortgeschrittenes Magenkarzinom

\section{2}

Klassifikation des Magenkarzinoms (Einteilung nach Siewert)

2.3 Epidemiologie des Magenkarzinoms

3. 
4.

Therapie des Magenkarzinoms

5.

6. Chemotherapie 
8.

Ergebnisse

8.1

Alter und Geschlecht und nach präoperativer Chemotherapie relativ zum UICC-Stadium computertomographischer Diagnostik

9. 

bei endoskopischer und computertomographischer Diagnostik

10.

11. 


\section{Einleitung und Fragestellung}

Seit Veröffentlichung der vielversprechenden Ergebnisse der MAGIC-Studie („Medical Research Council Adjuvant Gastric Infusional Chemotherapy Trial“) von Cunningham et al. (2006) ist allgemein anerkannt, dass mit einem perioperativem Chemotherapiekonzept das Outcome von Patienten mit lokal fortgeschrittenem Magenkarzinom signifikant verbessert werden kann. Neben einer präoperativen Remission des Primärtumors mit verbesserter Resektabilität kann eine frühe Elimination okkulter Mikrometastasen als ursächlich für ein signifikant höheres Gesamtüberleben im Vergleich zu alleiniger chirurgischer Therapie betrachtet werden. Als weiterer prognostischer Faktor zeigt sich in mehreren japanischen Studien das Ausmaß der Tumorresektion, insbesondere die Anzahl der entfernten Lymphknoten. Nach Sano et al. (2004) ist dabei eine große Anzahl entfernter Lymphknoten unter Einbeziehung des ersten und zweiten Drainagekompartiments bei adäquater chirurgischer Expertise mit einem Prognosevorteil hinsichtlich des postoperativen Gesamtüberlebens verbunden.

In einer Kombination aus perioperativer Chemotherapie und radikaler chirurgischer Tumorresektion zeigt sich ein vielversprechender Ansatz in der Therapie des lokal fortgeschrittenen Magenkarzinoms.

Zwischen November 2007 und Juli 2010 wurde in der Universitätsklinik der GeorgAugust-Universität Göttingen bei 20 Patienten mittels Ösophagogastroduodenoskopie (ÖGD) bei gleichzeitiger Biopsie aus suspekten Tumorarealen ein Adenokarzinom des Magens diagnostiziert. Anschließend wurden 17 dieser Patienten einer präoperativen Chemotherapie vor chirurgischer Tumorresektion unterzogen. In 15 von 17 Fällen konnte abschließend eine adjuvante Chemotherapie durchgeführt werden. 3 Patienten wurden nach Erstdiagnose in palliativer Intention behandelt. Die Aufarbeitung und Analyse der Behandlungsdaten erfolgte retrospektiv anhand der Patientenakten. Die Zusammenstellung der Patientenkohorte erfolgte konsekutiv mit dem Tag der Gastrektomie als Bezugsdatum.

Die vorliegende Arbeit verfolgt das Ziel, unter retrospektiver Betrachtung (medianes Follow-up 699 Tage/ mittleres Follow-up 507 Tage) den Behandlungsverlauf und - 
erfolg der perioperativen Chemotherapie dieser Patienten in der klinischen Routine zu bewerten und mit unter Studienbedingungen gewonnenen Parametern der MAGICArbeitsgruppe um Cunningham zu vergleichen (Cunningham et al. 2006). Dabei sollen insbesondere Tumorremission unter neoadjuvanter Chemotherapie, Anzahl der präund postoperativen Therapiezyklen, zeitlicher Abstand zwischen einzelnen Therapieschritten sowie postoperative Überlebensraten beurteilt werden.

Durch gleichzeitige Erhebung prognostisch relevanter Operationsdaten wie dem Ausmaß der Lymphknotenresektion und der Operationsdauer soll dargestellt werden, ob sich innerhalb unseres Kollektivs ein weiterer Überlebensvorteil durch eine hohe Anzahl dissektierter Lymphknoten ergibt. Durch eine Beurteilung der Stagingpräzision im N-Stadium mithilfe von endoskopischem Ultraschall (EUS) und Computertomographie (CT) sollen Rückschlüsse auf die präoperative Diagnostik des Lymphknotenbefalls gezogen werden.

Dabei gewonnene Erkenntnisse sollen international publizierten Daten gegenübergestellt werden. 


\section{Pathologie}

2.1 Das Magenkarzinom als neoplastische Läsion der Magenschleimhaut

Um ein Magenkarzinom handelt es sich bei allen malignen epithelialen Tumoren der Magenschleimhaut mit invasivem Wachstum und meist drüsiger Differenzierung.

Der Großteil der Magenkarzinome wird im Antrum diagnostiziert (30\%), gefolgt von Korpus und Fundus (20\%) und Kardia (15\%). Die verbliebenen 35\% erstrecken sich über mehr als einen Abschnitt (Riede et al. 2004).

Abhängig von der Infiltrationstiefe des Magenkarzinoms lässt sich zwischen Frühkarzinomen und fortgeschrittenen Karzinomen differenzieren.

\subsubsection{Magenfrühkarzinom}

Das Magenfrühkarzinom beschränkt sich in seiner Ausdehnung auf Mukosa (M-Typ) oder Submukosa (SM-Typ), während tiefer gelegene Schichten nicht infiltriert sind (Yasuda 2006). Trotz der geringen Eindringtiefe können in diesem Stadium bereits bei $30-50 \%$ aller Patienten Lymphknotenmetastasen diagnostiziert werden (Thomas 2006).

\subsection{Fortgeschrittenes Magenkarzinom}

Die Infiltrationstiefe des fortgeschrittenen Magenkarzinoms reicht mindestens bis zur Tunica muscularis oder bereits darüber hinaus, während in den meisten Fällen bereits lymphatische Metastasen der regionären Lymphknoten an kleiner und großer Kurvatur sowie am Milzhilus vorliegen (Riede et al. 2004, Böcker et al. 2008, Kumar et al. 2007). 


\subsection{Klassifikation des Magenkarzinoms}

\subsubsection{Histologisch (nach WHO)}

Nach dem Schema der Weltgesundheitsorganisation (WHO) wird der Tumor gemäß dem histologischen Bild eingeteilt in

- $\quad$ Adenokarzinome (65\% der Magenkarzinome) mit papillärer, tubulärer und muzinöser Subtypisierung

- $\quad$ Siegelringzellkarzinome $(25 \%$ der Magenkarzinome $)$ mit siegelringzellartigen Tumorzellen

- $\quad$ adenosquamöses Karzinom (5\% der Magenkarzinome)

- $\quad$ Plattenepithelkarzinom (selten)

- undifferenziertes Karzinom mit totalem Differenzierungsverlust der Tumorzellen.

\subsubsection{Histomorphologisch (Einteilung nach Laurén)}

Die histomorphologische Tumorbestimmung nach Laurén entscheidet zusammen mit der Tumorlokalisation über Resektionsabstände und Operationsverfahren (Öffner und Meyer 2008, Laurén 1965):

- intestinaler Typ (50\% der Fälle): der Tumor wächst häufig polypös und kann makroskopisch dem Borrmann Typ1 (s.u.) zugeordnet werden. Durch eine gute Abgrenzung gegen die Umgebung sind bei einer Resektion geringe Sicherheitsabstände $(\geq 5 \mathrm{~cm})$ erforderlich.

- diffuser Typ (40\% der Fälle): der Tumor ist schlecht differenziert mit infiltrativem Wachstum und früher Metastasierung. Generell sind große Resektionsabstände $(\geq 8 \mathrm{~cm}$ ) erforderlich.

- Mischtyp (10\% der Fälle): der Tumor kann weder intestinalem Typ noch diffusem Typ zugeordnet werden .

Die Zuordnung entweder zum intestinalen Typ oder zum diffusen Typ nach Laurén ist in der Praxis als entscheidender Parameter für die chirurgische Vorgehensweise bei 
der operativen Therapie des Magenkarzinoms zu werten. Während ein Magenkarzinom vom intestinalen Typ mit Lokalisation im distalen Magen in vielen Fällen einen schonenderen chirurgischen Eingriff im Sinne einer 4/5-Resektion des Magens mit D2-Lymphadenektomie (LAD) ermöglicht, ist beim diffusen Typ eine Gastrektomie bzw. transhiatal erweiterte Gastrektomie mit anschließender D2-LAD unumgänglich. Abhängig vom Typ sollten dabei oben genannte Resektionsmindestabstände eingehalten werden (Öffner und Meyer 2008, Laurén 1965).

\subsubsection{Makroskopisch (Einteilung nach Borrmann)}

Entsprechend der makroskopischen Wachstumsform lässt sich das Magenkarzinom vier verschiedenen Gruppen zuordnen (Öffner und Meyer 2008, Junginger et al. 2002, Borrmann 1928):

- Typ l: polypöse Karzinome mit exophytischem Wachstum

- Typ II: schlüsselförmiges, ulzeriertes Karzinom mit wallartiger, scharfer Begrenzung zur umgebenden Schleimhaut

- Typ III: ulzeriertes Karzinom mit unscharfer Abgrenzung zur umgebenden Schleimhaut

- Typ IV: diffus-infiltrierend wachsendes Karzinom, keine Ulzeration.

\subsubsection{Klassifikation von Kardiakarzinomen (Einteilung nach Siewert)}

Adenokarzinome des gastroösophagealen Übergangs (AEG) lassen sich nach Siewert (1987) entsprechend ihrer Lokalisation in drei Subtypen unterteilen (Siewert und Brauer 2009, Siewert et al. 2006):

- $\quad$ AEG I: die Haupttumormasse ist im distalen Ösophagus lokalisiert

- AEG II: die Haupttumormasse ist auf Höhe der Kardia lokalisiert

- AEG III: die Haupttumormasse ist unterhalb der eigentlichen Kardia lokalisiert.

Entsprechend der aktuellen Klassifikation von Wittekind et al. (2010) sind Adenokarzinome der Typen AEG I und II als Karzinome des distalen Ösophagus zu 
sehen, wodurch sich in der Behandlung dieser beiden Typen alternative Therapiekonzepte eröffnen (s. 9.1.2, S.65).

\subsection{Epidemiologie des Magenkarzinoms}

Adenokarzinome des Magens und des gastroösophagealen Übergangs stellen weltweit die vierthäufigste Tumorerkrankung dar und sind nach Lungenkrebs die zweithäufigste tumorbedingte Todesursache (Crew und Neugut 2006). Die Inzidenz des Magenkarzinoms unterliegt einer starken geographischen Variation, vor allem Japan und Korea zeigen im internationalen Vergleich eine hohe Anzahl jährlicher Neuerkrankungen (Yamamoto 2001, Ahn et al.1991).

Während die Inzidenz der distalen Adenokarzinome des Magens stetig abnimmt, lässt sich seit den achtziger Jahren des zwanzigsten Jahrhunderts eine deutliche Zunahme des proximalen Magenkarzinoms im Bereich der Kardia beobachten, von dem vor allem Männer betroffen sind (Kelley und Duggan 2003).

Das mittlere Erkrankungsalter beträgt für Frauen 75 Jahre, für Männer 70 Jahre.

Jährlich erkranken in Deutschland ca. 18.800 Menschen neu an einem Magenkarzinom, wobei es sich in 58,8\% der Fälle um Männer handelt.

Die Anzahl der Neuerkrankungen in Deutschland ist seit 1980 stark gesunken.

So erkrankten 2004 nur noch halb so viele Frauen an einem Magenkarzinom wie im Jahre 1980. Auch im männlichen Teil der Bevölkerung lässt sich im gleichen Zeitraum eine Abnahme um ca. 41\% feststellen (Robert-Koch-Institut 2008).

Die insgesamt abnehmende Inzidenz des Magenkarzinoms seit mehreren Jahrzehnten lässt erwarten, dass die Anzahl der Neuerkrankungen sowie die Mortalitätsraten weiterhin sinken.

\subsubsection{Geographische Variation der Inzidenz}

Abhängig von der geographischen Lage eines Landes variiert die Inzidenz des Magenkarzinoms signifikant. Im weltweiten Vergleich kann die Inzidenz fast um das 
dreißigfache ansteigen. Die jährliche Rate der Neuerkrankungen bei Männern liegt in Yamagata (Nordjapan) bei 176/100.000, während sie in Chiang-Mai (Thailand) nur 6,1/100.000 beträgt. Neben Japan ist Korea mit einer Inzidenz von 59,8/100.000 männlichen Einwohnern als Hochrisikoland zu nennen (Curado et al. 2007).

\begin{tabular}{|l|c|c|}
\hline \multicolumn{1}{|c|}{ Land, Region } & Männer & Frauen \\
\hline Japan, Yamagata & 176,0 & 87,0 \\
\hline Korea, Seoul & 59,8 & 31,4 \\
\hline China, Shanghai & 52,6 & 31,2 \\
\hline Italien, Genua & 35,6 & 24,8 \\
\hline Deutschland, Münster & 25,1 & 18,5 \\
\hline Costa Rica, San José & 24,5 & 14,9 \\
\hline Schweiz, Wallis & 20,6 & 9,7 \\
\hline Neuseeland, Wellington & 12,8 & 7,6 \\
\hline Thailand, Chiang-Mai & 6,1 & 5,1 \\
\hline
\end{tabular}

Tab.1: Internationaler Vergleich der Inzidenzraten des Magenkarzinoms (/100.000) (nach Curado et al. 2007)

Studien konnten belegen, dass sich bei Emigranten aus Regionen mit einem hohen Erkrankungsrisiko durch Ansiedlung in Ländern mit niedriger Inzidenz ebenfalls eine Reduktion des Erkrankungssrisikos einstellt (McMichael et al. 1980). Die Adaptation der Inzidenz und die Tatsache, dass nachfolgende Generationen von Emigranten das Erkrankungsrisiko des Gastlandes vollständig übernehmen (Parkin et al. 1997), spricht für die entscheidende Rolle von Ernährungsgewohnheiten und Lebensumständen bei der Pathogenese des Magenkarzinoms. Die Annahme einer erhöhten genetischen Prädisposition innerhalb bestimmter Bevölkerungsgruppen ist somit nicht anzunehemen. 


\section{4 Ätiologie des Magenkarzinoms}

Generell handelt es sich bei der Pathogenese eines Magenkarzinoms um ein multifaktorielles Geschehen (Vieth 2008).

Als „Karzinogen erster Ordnung“ (WHO) kann die Helicobacter-pylori-assoziierte Gastritis (Typ-B-Gastritis) aufgeführt werden, die in ihrer chronischen Verlaufsform über eine Mucosaatrophie zu einer intestinalen Metaplasie, einer präkanzerösen Läsion, führt. Da der Entzündungsverlauf durch zahlreiche exogene und genetische Faktoren beeinflusst wird, entwickeln nur wenige Patienten mit Helicobacter-pyloriInfektion anschließend auch ein Magenkarzinom. Es ist allgemein akzeptiert, dass eine Typ-B-Gastritis vor allem mit dem Magenkarzinom vom intestinalen Typ (nach Laurén ) assoziiert ist (Vieth 2008). Auch die autoimmune Gastritis (Typ-A-Gastritis) in Verbindung mit Mucosaatrophie und intestinaler Metaplasie kommt als Risikofaktor in Betracht. Der häufige Konsum von geräucherter, gepökelter sowie in Dosen konservierter Nahrung ist durch die gesteigerte Aufnahme von Nitraten mit einem erhöhten Karzinomrisiko verbunden. Nahrungsbakterien wandeln Nitrate zu Nitriten und Nitrosaminen mit kanzerogenem Potential um.

Andere aggressive Risikofaktoren exogenen Ursprungs sind Tabakkonsum, Alkoholabusus sowie Medikamente, bei denen vor allem nicht-steroidale Antirheumatika (NSAR) wie z.B. Acetylsalicylsäure und Diclofenac zu nennen sind.

Des Weiteren werden genetische Faktoren bei der Entstehung eines Magenkarzinoms in Betracht gezogen, wofür neben einer direkten Assoziation zwischen Blutgruppe A und dem Magenkarzinom vom diffusen Typ (nach Laurén) auch eine familiäre Häufung spricht. Das familiäre Magenkarzinom lässt sich in 30-40\% der Fälle auf eine Mutation des CDH1-Gens, welches für E-Cadherin kodiert, zurückführen. Bei dem Glykoprotein E-Cadherin handelt es sich um ein Zelladhäsionsmolekül, welches in seiner Funktion einer Tumorinvasion entgegenwirkt (Riede et al. 2004, Böcker et al. 2008, Kumar et al. 2007). 


\subsection{Metastasierung des Magenkarzinoms}

Die Metastasierung des Magenkarzinoms erfolgt vorwiegend auf lymphogenem Weg und per continuitatem. Bedingt durch die Drehung des Magens während der Embryonalentwicklung besteht die Möglichkeit, dass neben den Lymphknoten von Omentum majus und minus auch retroperitoneale Lymphknoten um den Truncus coeliacus metastatisch befallen werden (Sendler und Siewert 2000). In vereinzelten Fällen werden supraklavikuläre Lymphknoten metastatisch befallen, welche klinisch als verdickte "Virchow-Lymphknoten“ zu palpieren sind. Zur Strukturierung der gastralen Lymphabflusswege erfolgt eine Unterteilung in Kompartimente:

- Kompartiment 1 (D1): perigastrale Lymphknoten an Curvatura major und minor (in Abbildung 1 grün)

- Kompartiment 2 (D2): Lymphknoten von Truncus coeliacus, Ligamentum hepatoduodenale und des Milzhilus (in Abbildung 1 und 2 rot)

- Kompartiment 3 (D3): retroperitoneale Lymphknoten mit retropankreatischer und paraaortaler Lokalistation (in Abbildung 2 schwarz)

(Beer et al. 2007,Sendler et al 2002).

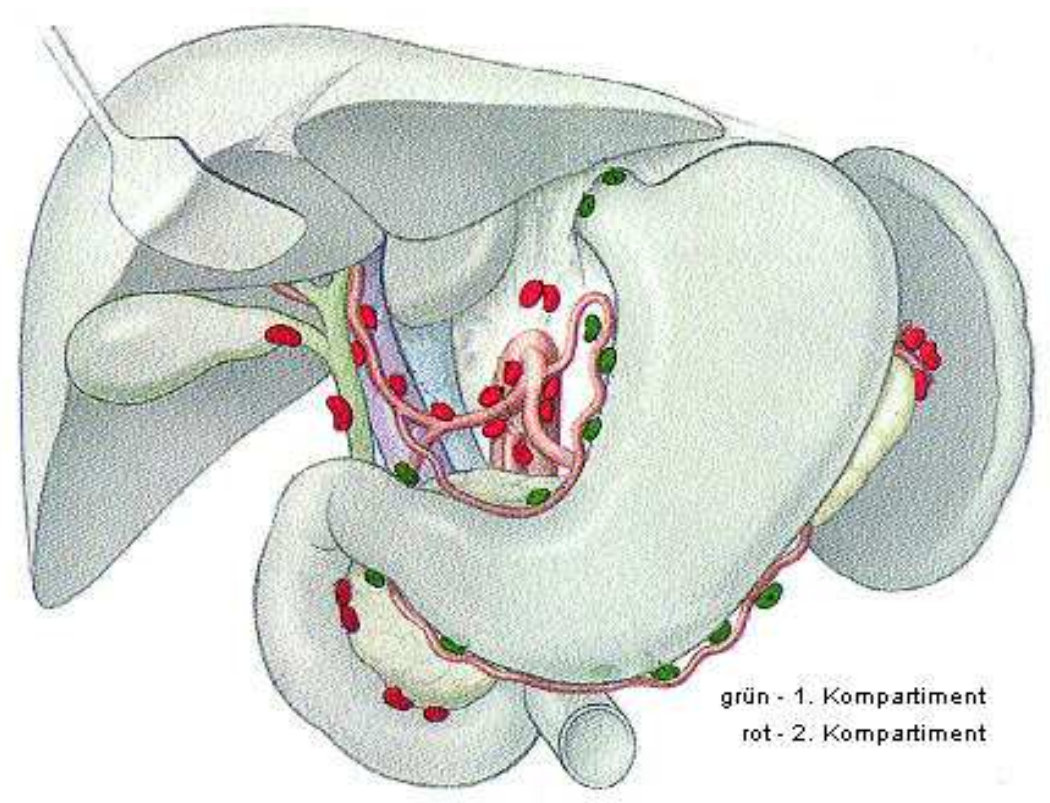

Abb. 1: Einteilung der Lymphknotengruppen des 1. und 2. Kompartiments (nach: Japanese Gastric Cancer Association 1998 , Abbildungsquelle: Prof. Dr. med. G. Ramadori) 


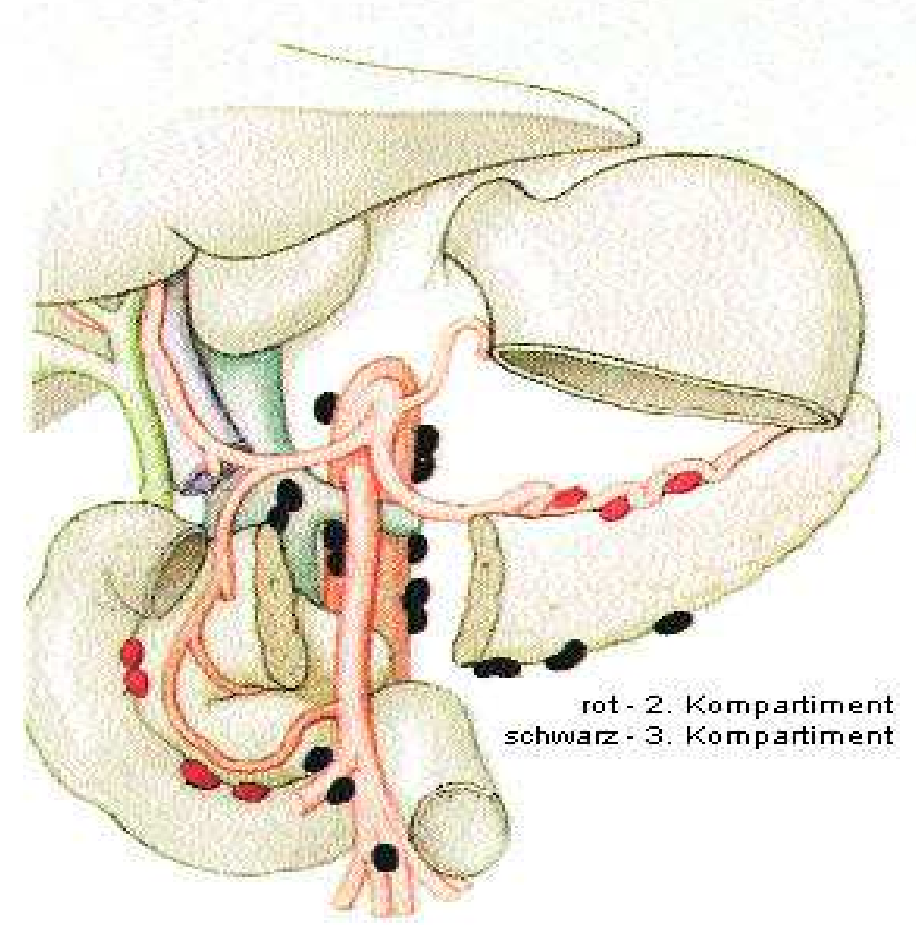

Abb.2: Lymphknotengruppen des 3. Kompartiments mit Anteilen des 2. Kompartiments (nach: Japanese Gastric Cancer Association 1998, Abbildungsquelle: Prof. Dr. med. G. Ramadori)

Die hämatogene Metastasierung des Magenkarzinoms erfolgt vorwiegend über den Pfortaderkreislauf mit der Folge einer metastatischen Infiltration der Leber (Beer et al. 2007).

Tumore der T-Kategorie 4 penetrieren per continuitatem die Leber, die Milz, das Colon transversum sowie das Pankreas. Bei weiblichen Patienten kann es zu intraperitonealen Abtropfmetastasen auf den Ovarien kommen, sogenannte „Krukenberg-Tumore“ (Riede et al. 2004). 


\subsection{Tumorstadien}

Im europäischen Raum und den Vereinigten Staaten erfolgt das Staging des Magenkarzinoms nach den Richtlinien der UICC (Union internationale contre le cancer) / AJCC (American Joint Committee on Cancer) mit Hilfe der TNMKlassifikation (Wittekind et al. 2002).

Zur prätherapeutischen Bewertung des Tumorstadiums wird dabei die Penetrationstiefe des Tumors durch die Magenwand (T-Kategorie), die Beteiligung von Lymphknoten (N-Kategorie) und das eventuelle Vorhandensein von Fernmetastasen (M-Kategorie) einbezogen (Tab.2). Durch histopathologische Untersuchung oder chirurgische Eingriffe verifizierte Daten werden mit einem vorangestellten „p“ gekennzeichnet (pTNM).

Alternativ kann ein Staging-System der „Japanese Research Society for Gastric Cancer" (JRSGC) verwendet werden. Die UICC/TNM-Klassifikation und die Japanese classification of gastric cancer (JCGC) unterscheiden sich vorwiegend in der Bewertung der befallenen Lymphknoten. Das JCGC-System bewertet die anatomische Verteilung metastatischer Lymphknoten, während sich die TNM-Klassifikation auf die Anzahl der befallenen Lymphknoten bezieht (Yamashita et al. 2008, Ichikura et al. 1999). 


\begin{tabular}{|l|l|}
\hline Tx & Primärtumor nicht zu beurteilen \\
\hline T0 & kein Anhalt für Primärtumor \\
\hline Tis & $\begin{array}{l}\text { Intraepithelialer Tumor ohne Infiltration der Lamina } \\
\text { propria } \\
\text { (= carcinoma in situ) }\end{array}$ \\
\hline T1 & $\begin{array}{l}\text { Tumor infiltriert Lamina propria (T1a) oder Submucosa } \\
\text { (T1b) }\end{array}$ \\
\hline T2 & $\begin{array}{l}\text { Tumor infiltriert Muscularis propria (T2a) oder Subserosa } \\
(\text { T2b) }\end{array}$ \\
\hline T3 & $\begin{array}{l}\text { Tumor penetriert Serosa ohne Infiltration benachbarter } \\
\text { Strukturen }\end{array}$ \\
\hline T4 & Tumor infiltriert benachbarte Strukturen \\
\hline Nx & Regionäre Lymphknoten nicht zu beurteilen \\
\hline N0 & Keine Lymphknotenmetastasen1 \\
\hline N1 & Metastasen in 1-6 regionären Lymphknoten \\
\hline N2 & Metastasen in 7-15 regionären Lymphknoten \\
\hline N3 & Metastasen in mehr als 15 regionären Lymphknoten \\
\hline M0 & keine Fernmetastasen \\
\hline Fernmetastasen \\
\hline
\end{tabular}

Tab. 2: TNM-Klassifikation des Magenkarzinoms nach UICC (2002)

${ }^{1}$ Bestimmung von pNO erfordert regionäre Lymphadenektomie und histologische Untersuchung von > 15 Lymphknoten 


\begin{tabular}{|c|c|c|c|}
\hline Stadium 0 & pTis & pNO & MO \\
\hline Stadium IA & pT1 & pNO & M0 \\
\hline \multirow[t]{2}{*}{ Stadium IB } & pT1 & pN1 & M0 \\
\hline & $\mathrm{pT} 2 \mathrm{a} / \mathrm{b}$ & $\mathrm{pNO}$ & Mo \\
\hline \multirow[t]{3}{*}{ Stadium II } & pT1 & pN2 & M0 \\
\hline & $\mathrm{pT} 2 \mathrm{a} / \mathrm{b}$ & pN1 & M0 \\
\hline & pT3 & pNO & M0 \\
\hline \multirow[t]{3}{*}{ Stadium IIla } & $\mathrm{pT} 2 \mathrm{a} / \mathrm{b}$ & pN2 & M0 \\
\hline & pT3 & $\mathrm{pN} 1$ & M0 \\
\hline & pT4 & pNO & M0 \\
\hline Stadium IIIb & pT3 & pN2 & M0 \\
\hline \multirow[t]{3}{*}{ Stadium IV } & pT1-3 & pN3 & M0 \\
\hline & pT4 & pN1-3 & MO \\
\hline & Jedes pT & Jedes pN & M1 \\
\hline
\end{tabular}

Tab.3: Stadiengruppierung beim Magenkarzinom nach UICC (2002)

Als entscheidende Faktoren für die Verlaufsprognose müssen am Tumorgewebe bestimmt werden (Menges 2004):

die R-Kategorie (unter Beurteilung der Schnittränder)

R0 kein Residualtumor (makroskopische und mikroskopische Tumorfreiheit)

R1 mikroskopischer Resdiualtumor (Schnittränder nicht tumorfrei)

R2 makroskopischer Residualtumor 
der Differenzierungsgrad (nach UICC)

G1 gut differenziertes Karzinom

G2 mäßig differenziertes Karzinom

G3 schlecht differenziertes Karzinom

G4 nicht differenziertes Karzinom

Gx Differenzierungsgrad kann nicht bestimmt werden

Einteilung der Differenzierung nach Laurén ( $\rightarrow$ s. 2.2.2 ).

In die vorliegende Arbeit einbezogene Patientendaten zu TNM-Klassifikation und Tumorstadium wurden entsprechend der UICC-Vorgaben aus dem Jahr 2002 erhoben. Eine Weiterentwicklung des TNM-Klassifikationssystems, in der sich wesentliche Änderungen zur T- und N-Kategorie ergeben, wurde im Januar 2010 von Wittekind und Meyer publiziert. T-Kategorie 1 unterteilt sich demnach in T1a-Karzinome mit maximaler Invasionstiefe bis zur Muscularis mucosa und T1b-Karzinome, bei denen bereits eine Infiltration der Submukosa nachzuweisen ist. T2-Karzinome zeigen nach den neuen Richtlinien einen Tumorbefall der Muscularis propria während sich T3-Karzinome bereits bis in die Subserosa ausweiten. Einer T4a-Kategorie werden Tumore mit Perforation der Serosa zugeordnet. Tumore der Kategorie T4b befallen benachbarte Strukturen. Weitere Änderungen zeigen sich in der Beurteilung der lymphogenen Metastasierung: Kategorie N1 umfasst 1 bis 3, Kategorie $\quad \mathrm{N} 2 \quad 4 \quad$ bis 6 und Kategorie N3 7 oder mehr regionäre Lymphknotenmetastasen. Den Umstrukturierungen der $\mathrm{T}$ - und $\mathrm{N}$-Kategorie entsprechend wurde auch die Stadiengruppierung umgestaltet (Wittekind und Meyer 2010). 


\section{Diagnostik des Magenkarzinoms}

\subsection{Klinische Symptomatik und Verdachtsmomente}

Die klinische Symptomatik des Magenkarzinoms stellt sich zumeist erst nach längerem Bestehen der Erkrankung ein und ist insgesamt unspezifisch, so dass zwei Drittel der Magenkarzinome in einem bereits fortgeschrittenen Stadium diagnostiziert werden. Nach einer Studie von Wanebo et al. (1993) an insgesamt 18.363 Patienten mit Magenkarzinom besteht die Symptomatik typischerweise aus Gewichtsverlust $(61,6 \%)$, Bauchschmerzen $(51,6 \%)$, Übelkeit $(34,3 \%)$, Erbrechen $(32,0 \%)$ und verfrühtem Sättigungsgefühl (17,5\%) (Wanebo et al. 1993).

Das Erbrechen von Stuhl und Anzeichen eines lleus können zudem Hinweis auf eine tumorbedingte Verlegung des Gastrointestinaltraktes sein.

Als typische Stationen der Metastasierung können die supraklavikulären Lymphknoten auf der linken Körperseite (Virchow-Lymphknoten) sowie die Leber vergrößert sein (Englisch-Fritz et al. 2008).

Bei auffälliger Anamnese und körperlicher Inspektion mit Verdacht auf ein Magenkarzinom ist eine umfassende bildgebende Diagnostik unumgänglich.

\subsection{Bildgebende Diagnostik}

\subsubsection{Gastroskopie}

Initialer Schritt der apparativen Diagnostik ist eine umfassende Ösophagogastroduodenoskopie (ÖGD) mit gleichzeitiger Entnahme von 3-10 Biopsien aus suspekten Mukosaarealen (Messmann und Schlottmann 2001). Mit Hilfe dieser ersten Biopsien erfolgt die histomorphologische Zuordnung des Tumorgewebes gemäß der Klassifikation nach Laurén (1965). Neben der histologischen Diagnosesicherung liefert die umfassende Gastroskopie Aussagen über Lokalisation, 
oberflächliche Größe und die makroskopische Wachstumsform (nach Borrmann) des Karzinoms (Menges 2004, Möhler und Galle 2007). Die diagnostische Sensitivität der Gastroskopie beträgt bei Entnahme von mindestens 4 Biopsien bis zu 95\% (Öffner und Meyer 2008). Die genaue anatomische Zuordnung des Tumors mittels Gastroskopie ist besonders bei Karzinomen des gastroösophagealen Übergangs von Bedeutung, da hierbei die Lage des Tumors über das operative Konzept entscheidet (Sendler und Siewert 2000).

\subsubsection{Ausbreitungsdiagnostik (Staging)}

Bei Diagnose eines Magenkarzinoms im Rahmen der initialen Ösophagogastroduodenoskopie(ÖGD) schließt sich eine ausführliche Ausbreitungssdiagnostik (Staging) an. Zur genaueren Differenzierung des Tumorstadiums sollten dabei eine Sonographie des Abdomens und eine hochauflösende Computertomographie (CT)- Diagnostik zur Anwendung kommen. Um das Vorhandensein metastatischer Krukenberg-Tumore auszuschließen, ist bei Frauen das obere Becken in die CT-Untersuchung einzubeziehen (Menges 2004). Nach Leitlinien der Deutschen Krebsgesellschaft sollte außerdem eine RöntgenThorax-Aufnahme in 2 Ebenen angefertigt werden (Beer et al. 2007).

Um die Penetrationstiefe des Tumors in die Magenwand, also das T-Stadium, zu bestimmen, erfolgt anschließend der endoluminale Ultraschall (EUS) (Pfau und Chak 2002, Virányi et al. 2006). Studien belegen, dass die EUS mit einer diagnostischen Treffgenauigkeit von 78\% (Willis et al. 2000) der CT-Untersuchung bei der Bestimmung des T-Stadiums überlegen ist (Öffner und Meyer 2008, Moretó 2003). Gerade bei proximalen Magenkarzinomen bzw. Karzinomen des gastroösophagealen Übergangs sollte neben der endoluminalen Ultraschalluntersuchung im erweiterten Staging eine CT-Aufnahme des Thorax angefertigt werden, um eine Begutachtung der mediastinalen Lymphknoten zu ermöglichen und eventuelle Lungenmetastasen zu diagnostizieren (Möhler und Galle 2007). Die Notwendigkeit einer Skelettszintigraphie ergibt sich bei Befunden mit Hinweis auf eine skelettale Metastasierung wie Skelettschmerzen oder Auffälligkeiten im Blutbild, z.B. Anstieg der alkalischen Phosphatase (Drzezga et al. 2005). 
Sobald Tumoren die Serosa penetrieren oder überschreiten (T-Kategorie 3 oder 4) und ein neoadjuvantes Therapiekonzept geplant ist, ist im Anschluss eine erweiterte diagnostische Laparoskopie (EDL) indiziert (Feussner et al. 2003, Kriplani und Kapur 1991, Lehnert et al. 2002). In einer Studie von Feussner et al. (2003) konnten bei 111 Patienten mit T3/T4-Tumoren nach vorangehender bildgebender Diagnostik (EUS/US/CT) in 40,5\% das Tumorstadium mit Hilfe der EDL korrigiert werden, wodurch sich wesentliche Änderungen des Therapiekonzepts ergaben. Auch Meyer und Wilke sehen eine adäquat chirurgisch durchgeführte Laparoskopie als bedeutendes bildgebendes Diagnosemittel zur Stagingpräzisierung bei T3/T4Tumoren oder Magenkarzinomen vom diffusen Typ nach Laurén. Gerade die Exploration subkapsulärer Lebermetastasen und lokalisierter Peritonealkarzinosen kann dabei therapeutische Konsequenzen nach sich ziehen (Meyer und Wilke 2011).

Schematisiert lässt sich die klinische Leitlinie in Form eines Diagnose-Algorithmus verdeutlichen (Abb. 3).

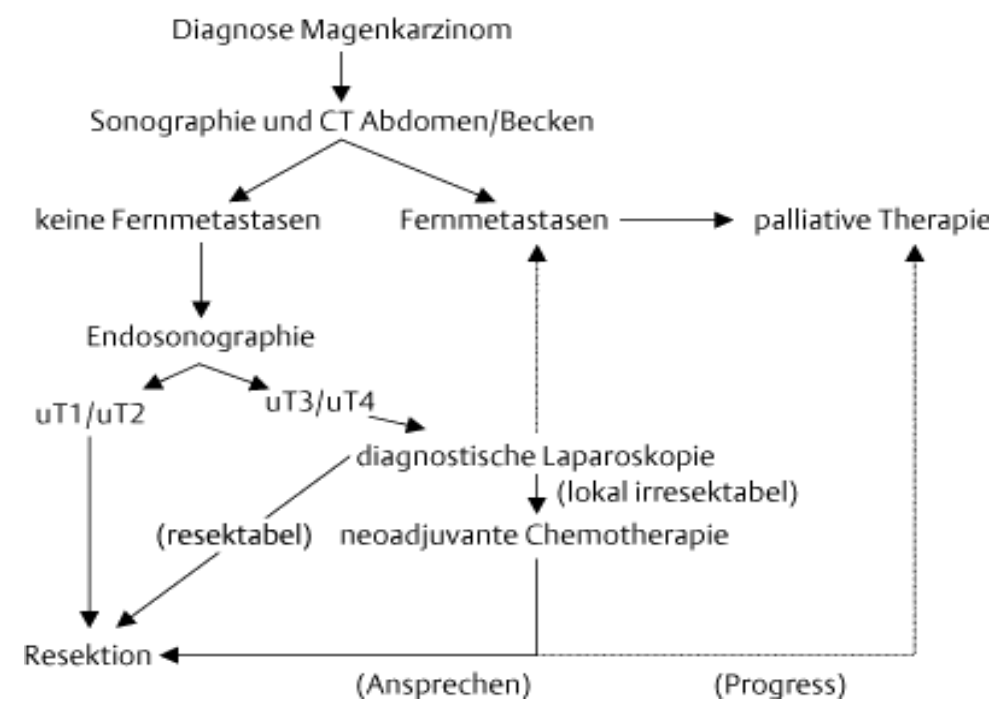

Abb.3: Diagnose-Algorithmus zum Magenkarzinom (aus Sendler und Siewert, 2000, S.325 ) 


\section{Therapie des Magenkarzinoms}

\subsection{Chirurgisch}

Es ist allgemein anerkannt, dass ausschließlich die vollständige Resektion des Tumors (R0 nach UICC) kombiniert mit einer Lymphadenektomie des zugehörigen Abflussgebietes als potentiell kurativ angesehen werden kann ( Hur und Park 2009, Dtsch. Gesellschaft für Chirurgie / Dtsch. Krebsgesellschaft 2003, Möning et al. 2007). Ein limitiertes chirurgisches Verfahren ohne Resektion des möglicherweise betroffenen Lymphabflussgebietes ist einzig bei Mukosakarzinomen indiziert, da in diesem Fall die Wahrscheinlichkeit einer lymphatischen Metastasierung unter 5\% liegt (Feussner 2004, Schröder et al. 2001). Die Ausdehnung der Läsion entscheidet im Falle eines Mukosakarzinoms über das chrirugische Vorgehen: Bis zu einem Läsionsdurchmesser von $2 \mathrm{~cm}$ ist eine Entfernung mittels endoskopischer Mukosaresektion (EMR) oder laparoskopischer Magenwandteilresektion möglich, bei größeren Mukosakarzinomen sollte eine konventionelle Magenwandresektion erfolgen (Minoru et al. 2000, Möhler und Galle 2007).

Wird im präoperativen Staging ein Tumorstadium diagnostiziert, bei dem von einer Lymphknotenmetastasierung auszugehen ist (ab Stadium Ib nach UICC, 2002), aber eine R0-Resektion durchgeführt werden kann, ist eine offene Resektion mit Lymphadenektomie angezeigt (Möning et al. 2007). Über die Art des operativen Vorgehens entscheidet die anatomische Lage des Primärtumors. Um sich dabei eines Operationsschemas mit definierten Resektionsgrenzen zu bedienen, wird der Magen gemäß seiner Gefäßversorgung in Drittel eingeteilt. Die Gastrektomie bei Karzinomen des proximalen Magendrittels erfolgt transhiatal erweitert unter Resektion des distalen Ösophagus. Während bei Tumoren mit Lokalisation im mittleren Magendrittel eine totale Gastrektomie erfolgt, ist bei Tumorlokalisation im distalen Drittel eine subtotale distale Magenresektion indiziert (Sendler und Siewert 2000). Die einzuhaltenden intraluminären Resektionsabstände sollten dabei beim Karzinom vom intestinalen Typ nach Laurén mindestens $5 \mathrm{~cm}$ und beim diffusen Typ mindestens $8 \mathrm{~cm}$ betragen (Sendler et al. 2002, Dtsch. Gesellschaft für Chirurgie / Dtsch. Krebsgesellschaft 
2003). Über das extraluminäre Resektionsausmaß, vor allem über die systematische Lymphadenektomie, herrscht allgemein Uneinigkeit. Bei der D1-Lymphadenektomie beschränkt sich die Lymphknotendissektion auf das 1. Kompartment, bei der D2Lymphadenektomie werden zusätzlich die Lymphknoten des 2. Kompartments reseziert (Zittel 2002). Im direkten Vergleich zwischen D1- und D2-Lymphadenektomie ergaben zwei prospektive Studien aus den Niederlanden und Großbritannien, dass zwischen beiden Strategien der Lymphknotenresektion in der 5-JahresÜberlebensrate keine signifikanten Unterschiede festzustellen sind (Cuscheri et al. 1999, Bonenkamp et al. 1999). In beiden Studien wird zudem dargelegt, dass eine D2Resektion im erhöhten Maße mit postoperativen Komplikationen verbunden ist. Diese erhöhte Komplikationsrate ist vor allem auf die hohe Frequenz an Splenektomien und Pankreaslinksresektionen zurückzuführen. Nicht zu vernachlässigen ist hierbei, dass die persönliche und institutionelle Expertise der verschiedenen Operateure durch die Vielzahl an teilnehmenden Zentren nicht hinreichend beurteilt werden kann (Roukos 2000, Van de Velde 2005). Dem gegenüber stehen verschiedene japanische Studien und die deutsche Magenkarzinomstudie, die bei geringer Morbidität und Mortalität einen Prognosevorteil nach D2-Lymphadenektomie sehen (Fujii et al. 2010, Sano et al. 2004, Sano et al. 2002, Siewert et al. 1998).

\subsection{Multimodale Therapiekonzepte}

\subsubsection{Adjuvante Therapie}

Ziel der postoperativen adjuvanten Chemo- oder Radiochemotherapie ist eine Verbesserung der Überlebensprognose des Patienten nach erfolgreicher R0Resektion durch Senkung der Rezidivrate (Schmidt-Matthiesen und Bechstein 2005). Die Wirksamkeit der adjuvanten Chemotherapie galt lange Zeit als umstritten. In der amerikanischen Intergroup-Studie 0116 (MacDonald et al. 2001) konnte jedoch belegt werden, dass bei Patienten, die einer postoperativen Radiochemotherapie unterzogen wurden, das Gesamtüberleben verglichen mit Patienten nach alleiniger Resektion signifikant verbessert wurde. Obwohl der Intergroup-Studie 0116 methodische 
Schwierigkeiten vorgeworfen wurden, konnte eine letzte Metaanalyse die Studienergebnisse zugunsten des adjuvanten Therapiekonzeptes untermauern (Zhao und Fang 2008). Aufgrund der häufigen therapiebedingten Komplikationen und hohen Toxizität der adjuvanten Chemo- oder Radiochemotherapie sowie des hohen operativen Standards in Deutschland (systematische D2-Lymphadenektomie) ist eine adjuvante Therapie des R0-resezierten Magenkarzinoms trotzdem nicht allgemein indiziert, kann aber in Einzelfällen, vor allem bei Hochrisikopatienten, zur Diskussion stehen (Möhler und Galle 2007, Kollmannsberger et al. 2005).

\subsubsection{Neoadjuvante Therapie}

Ziele eines neoadjuvanten Therapieansatzes sind ein „Down-Sizing“, also eine Umfangsminderung des Primärtumors, um eine vollständige Resektion zu ermöglichen, sowie die Elimination okkulter Mikrometastasen zur Senkung der postoperativen Rezidivrate (Becker et al. 2003). Neben einer intakten Blutversorgung und den komplett erhaltener Lymphabflusswegen kann auch der bessere Allgemeinzustand des Patienten als Argument für eine präoperative Chemotherapie gesehen werden (Sendler und Siewert 2000). Aktuell veröffentlichte Ergebnisse der britischen MAGIC-Studie (Cunningham et al. 2006) und einer französischen Multicenter-Studie (Boige et al. 2007) zeigen einen deutlichen Vorteil in der Überlebensrate perioperativ (MAGIC-Studie) bzw. präoperativ (Boige et al.2007) chemotherapierter Patienten verglichen mit einer ausschließlich chirurgisch behandelten Patientengruppe. Im direkten Vergleich zwischen neoadjuvant und ausschließlich chirurgisch therapierten Patienten kann dabei kein signifikanter Anstieg der postoperativen Morbidität und Mortalität festgestellt werden (Cunningham et al. 2007, Biffi et al. 2010).

Die Ergebnisse dieser Studien belegen, dass ein Ansprechen auf eine perioperative Chemotherapie als signifikanter Surrogatmarker für die Beurteilung der postoperativen Überlebenschancen herangezogen werden kann. 


\subsection{Palliatives Therapiekonzept}

Bei Patienten mit bereits metastasiertem Magenkarzinom, bei denen eine chirurgische Komplettresektion des Primärtumors unmöglich ist (Stadium IIIb, IV nach UICC, 2002), sollten palliativ mit einer systematischen Chemotherapie behandelt werden (Vanhoefner und Schleuchner 2004). Voraussetzung dafür ist ein stabiler Allgemeinzustand des Patienten (Menges 2004). Die palliative Chemotherapie dient der Beseitigung lokaler Komplikationen bei Erhaltung einer möglichst hohen Lebensqualität (Sendler und Siewert 2000). In verschiedenen Studien konnte die Überlegenheit der palliativen Chemotherapie gegenüber dem „best supportive care“ hinsichtlich Lebensqualität und Überlebensdauer dargestellt werden (Gimelius et al. 1997,Pyrhonen et al. 1995).

Indikationen für eine chirurgische Palliation stellen funktionell wirksame Stenosen, akute Blutungen und Perforationen dar (Öffner und Meyer 2008). Die Wiederherstellung der Speisepassage im Falle einer Stenose kann ( bei gegebener Operabilität) über eine Bypass-Anastomose, eine palliative Gastrektomie oder über die Platzierung eines metallischen Stents erfolgen (Öffner und Meyer 2008, Hyoung et al. 2009, Jeong et al. 2004).

\subsection{Therapie des Magenkarzinoms in Japan}

\subsubsection{Präventive Screening-Programme}

Wie Tabelle 1 zeigt, ist in der japanischen Bevölkerung eine besonders hohe Inzidenz des Magenkarzinoms festzustellen. Mit 15,8\% (in 2004) ist Magenkrebs Todesursache Nummer eins unter allen Krebserkrankungen in Japan. Da die Überlebensprognose mit dem Fortschritt der Erkrankung abnimmt, versucht man bereits seit 1960, über landesweite Screening-Programme den Anteil der Frühdiagnosen des Magenkarzinoms zu erhöhen. Mittlerweile nehmen jährlich über 6 Millionen Japaner im Alter von über 40 Jahren an Screening-Programmen teil, bei denen vor allem die Photofluorographie, deren Sensitivität bei Massen-Screenings 70- 
90\% erreicht, als diagnostisches Hilfsmittel zur Anwendung kommt (Tsubono und Hisamichi 2008). Andere Screeningmethoden wie Endoskopie, Serum-PepsinogenTests oder Helicobacter-pylori-Antikörper-Tests erwiesen sich in einer aktuellen Studie für breit angelegte Untersuchungen als ungeeignet (Hamashima et al. 2008).

\subsubsection{Radikale Chirurgie}

Es ist weltweit akzeptiert, dass eine radikale chirurgische Magenresektion bei einem fortgeschrittenen Karzinom die einzige Therapieoption mit kurativem Potential darstellt. Häufig findet bereits zu einem frühen Zeitpunkt im Krankheitsverlauf eine lymphogene Metastasierung statt, deren Ausmaß das chirurgische Vorgehen maßgeblich beeinflusst. Während in Europa allgemein Uneinigkeit bezüglich des Umfangs der Lymphadenektomie herrscht, gehören in Japan D2-Resektionen bereits seit 1960 zur chirurgischen Routine (Fujii et al. 2010, Sano et al. 2002, Sano et al. 2004). Neben den Lymphknoten entlang der großen und kleinen Kurvatur, auf die sich die eingeschränkte D1-Lymphadenektomie begrenzt, werden dabei die Lymphknoten nahe der großen Magengefäße und des Truncus coeliacus reseziert (Möning et al. 2005). Wird in der westlichen Welt im klinisch-chirurgischen Sprachgebrauch von einer „erweiterten Lymphknotenresektion“ gesprochen, ist damit vorwiegend eine D2Resektion gemeint. In Japan versteht man darunter eine D2-Resektion mit zusätzlicher Exzision der para-aortalen Lymphknoten, wie sie dort in spezialisierten Zentren durchgeführt wird. Obwohl eine Studie von Sano et al. (2004) belegt, dass sich durch eine Resektion des 2. Kompartiments mit zusätzlicher Entfernung der paraaortalen Lymphknoten keine Verbesserung der 5-Jahres-Überlebensrate ergibt, kann diese Abhandlung als Richtwert des chirurgisch Möglichen dienen. „Eine Gastrektomie mit D2-Lymphadenektomie kann von spezialisierten Chirurgen bei Patienten mit niedrigem operativen Risiko sicher durchgeführt werden. Eine para-aortale Lymphadenektomie kann ohne Erhöhung der chirurgischen Hauptkomplikationen angeschlossen werden“ (Sano et al. 2004, S.2773). Ähnliche Ergebnisse zeigt eine aktuelle Metaanalyse von Wang et al. (2010).

Dabei müssen die prognostisch hoch relevanten Rollen der chirurgischen Expertise und des „workload volumes“ der behandelnden Zentren unterstrichen werden. Beispielhaft hierfür ist die Mortalitätsrate bei Gastrektomie im Vergleich zwischen 
westlichen Ländern (5-16\%) und Japan (<2\%), wo jährlich annähernd 100.000 Magenkarzinome diagnostiziert und therapiert werden, anzuführen (Sano et al. 2004, Fuji et al. 1999).

\section{Prognose von Patienten mit Magenkarzinom}

Die Überlebensprognose des Magenkarzinoms zeigt eine Abhängigkeit vom Tumorstadium zum Zeitpunkt der Operation. Bei Chirurgie unter kurativem Ansatz können 5-Jahres-Überlebensraten von 80\% im Stadium I, 40\% im Stadium II, 30\% im Stadium III und maximal 5\% im Stadium IV erreicht werden (Wilke et al. 2006) . Da ca. $70 \%$ der Magenkarzinome erst in einem weit fortgeschrittenen Krankheitsstadium (Stadien III und IV) entdeckt werden, ist die 5-Jahres-Überlebensrate aller Patienten als schlecht zu beurteilen (Sendler et al. 2000). Auf den entscheidenden Überlebensvorteil bei einer Früherkennung und die Notwendigkeit präventiver Screeningmaßnahmen nach japanischem Vorbild weisen die relativ guten 5-JahresÜberlebensraten der Stadien I und II hin.

Eine Studie von Desai et al. (2004) zeigt, dass sich die Prognose nach kurativer Magenkarzinomchirurgie über einen Zeitraum von ca. 20 Jahren (1982-2001) kontinuierlich verbessert hat. Ursächlich dafür geben die Autoren zum einen die optimierte Technik und vermehrte Anwendung der Endoskopie, zum anderen die Weiterentwicklung chirurgischer Strategien, insbesondere bezüglich zum Ausmaß der Lymphadenektomie, an.

\section{MAGIC-Studie}

Bei der MAGIC-Studie („Medical Research Council Adjuvant Gastric Infusional Chemotherapy Trial“, Cunningham et al. 2006) handelt es sich um eine multizentrische, randomisierte Phase-III-Studie zur neoadjuvanten Chemotherapie des 
operierbaren Magenkarzinoms im direkten Vergleich zu einer rein chirurgischen Therapie mit dem Zielsetzung, durch einen perioperativen Therapieansatz das Überleben der Patienten zu verbessern.

Bei dieser zweiarmigen Studie wurden Patienten mit lokal fortgeschrittenen Magenkarzinomen randomisiert zwei Gruppen zugeteilt. Während die eine Patientengruppe (253 Patienten / S-Gruppe) ausschließlich einer Resektion des Tumors unterzogen wurde, erhielt die zweite Patientengruppe (250 Patienten / CSCGruppe) zusätzlich präoperativ und postoperativ jeweils 3 Zyklen Epirubicin/Cisplatin/5-Fluorouracil (ECF). Bei den neoadjuvant therapierten Patienten konnte dabei ein signifikantes Down-Sizing und Down-Staging mit erhöhten Resektionsraten sowie ein deutlich verbessertes progressionsfreies Überleben (Hazard ratio für Progression 0,66; 95\% Konfidenzintervall 0,53-0,81; $\mathrm{p}<0,001$ ) und Gesamtüberleben festgestellt werden.

Bei einer mittleren Nachbeobachtungszeit von 4 Jahren betrug die 5-JahresÜberlebensrate in der perioperativ chemotherapierten Gruppe 36\%, während sie in der ausschließlich chirurgisch behandelten Gruppe bei $23 \%$ lag, was einem hoch signifikanten Überlebensvorteil von 13\% entspricht ( Hazard ratio für Tod 0,75; 95\% Konfidenzintervall, 0,60-0,93; $p=0,009)$. Die mediane Tumorgröße wurde durch die präoperative Chemotherapie signifikant verringert $(3 \mathrm{~cm}$ CSC-Gruppe vs. $5 \mathrm{~cm}$ S-Gruppe, $p<0,001)$.

Sowohl in der Häufigkeit der postoperativen Komplikationen (46\% CSC-Gruppe vs. 45\% S-Gruppe) als auch in der postoperativen Letalität (5,6\% CSC-Gruppe vs. 5,9\% S-Gruppe, 30 Tage postoperativ) konnten keine signifikanten Unterschiede ausgemacht werden (Cunningham et al. 2006).

Kritiker werfen der MAGIC-Studie Fehler in der methodischen und praktischen Vorgehensweise vor. Vor allem die Inhomogenität der Einschlusskriterien (Magen-, Kardia- und distale Ösophaguskarzinome), der lange Rekrutierungszeitraum (Juli 1994 - April 2002) und ungenaue Vorgaben bezüglich Staging und Ausmaß der Lymphadenektomie werden dabei angeführt (Siewert 2006, Möhler und Galle 2007).

Obwohl diese Einwände durchaus als berechtigt anzusehen sind, belegt ein absoluter Überlebensvorteil von $13 \%$ die Wirksamkeit der neoadjuvanten Chemotherapie und lässt Vorhersagen auf ihre Position als zukünftigen therapeutischen Standard zu. 


\section{Patienten und Methoden}

\subsection{Zusammensetzung des Kollektivs}

Zwischen November 2007 und Juli 2010 wurde im Zentrum für Innere Medizin des Universitätsklinikums der Georg-August-Universität Göttingen bei 20 Patienten ein lokal fortgeschrittenes Karzinom des Magens diagnostiziert. Anschließend wurde bei 17 dieser 20 Patienten eine präoperative Chemotherapie vor chirurgischer Tumorresektion durchgeführt. In 15 Fällen konnte abschließend eine adjuvante Chemotherapie durchgeführt werden. 3 Patienten, deren Daten in dieser Arbeit keine weitere Berücksichtigung finden, wurden nach der Erstdiagnose unter palliativer Betrachtung therapiert. Die Verlaufskontrolle der operativ behandelten Patienten erfolgte unter Anwendung von endoluminaler Ultraschalluntersuchung (EUS), Abdomensonographie, Computertomographe sowie Ösophagogastroduodenoskopie (ÖGD) (s. 7.Patienten und Methoden). Bei der weiteren postoperativen Nachbeobachtung wurde ein Intervall von 3 Monaten angestrebt.

Die Aufarbeitung und Analyse der Behandlungsdaten erfolgte retrospektiv (medianes Follow-up 699 Tage/ mittleres Follow-up 507 Tage) anhand der Patientenakte.

Einschlusskriterium für das Patientenkollektiv dieser retrospektiven Studie war eine in der Abteilung Gastroenterologie und Endokrinologie (Leitung Prof. Dr. Giuliano Ramadori) mittels Gastroskopie und Histologie gesicherte Magenkarzinomdiagnose vor anschließender präoperativer Chemotherapie. Die Patientenakquise erfolgte konsekutiv mit dem Operationstag als Bezugsdatum.

Von der Studie auszuschließen waren rein operativ oder palliativ behandelte Patienten, deren Therapieansatz nicht in Anlehnung an das in der MAGIC-Studie von Cunningham et al. (2006) präsentierte neoadjuvante Behandlungskonzept erfolgen konnte. Ein weiteres Ausschlusskriterium waren unvollständig vorliegende Krankenakten, um die Erhebung aller statistisch benötigten Daten zu gewährleisten. Im nachfolgenden beschriebene Statistiken und Ergebnisse beziehen sich auf dieses Kollektiv. 


\subsection{Durchführung und Planung der perioperativen Chemotherapie}

\subsubsection{Erstdiagnose und präoperatives Staging}

Bei bestehendem Verdacht auf einen Tumor des oberen Gastrointestinaltraktes wurde bei 13 Patienten nach vorhergehender Aufklärung und Einverständniserklärung eine Ösophagogastroduodenoskopie (ÖGD) bei gleichzeitiger Entnahme von 3-10 Biopsien aus suspekten Arealen der Magenmukosa durchgeführt. Bei 4 Patienten (davon 2 mit Linitis plastica) erfolgte lediglich eine ÖGD ohne Gewebeentnahme. Die Ursache für die fehlende Biopsie konnte retrospektiv nicht ermittelt werden. Bei dem verwendeten Gastroskopiegerät handelte es sich um das Evis Exera II (GIF Q165) Videogastroskop der Marke Olympus GmbH (Hamburg, Deutschland).

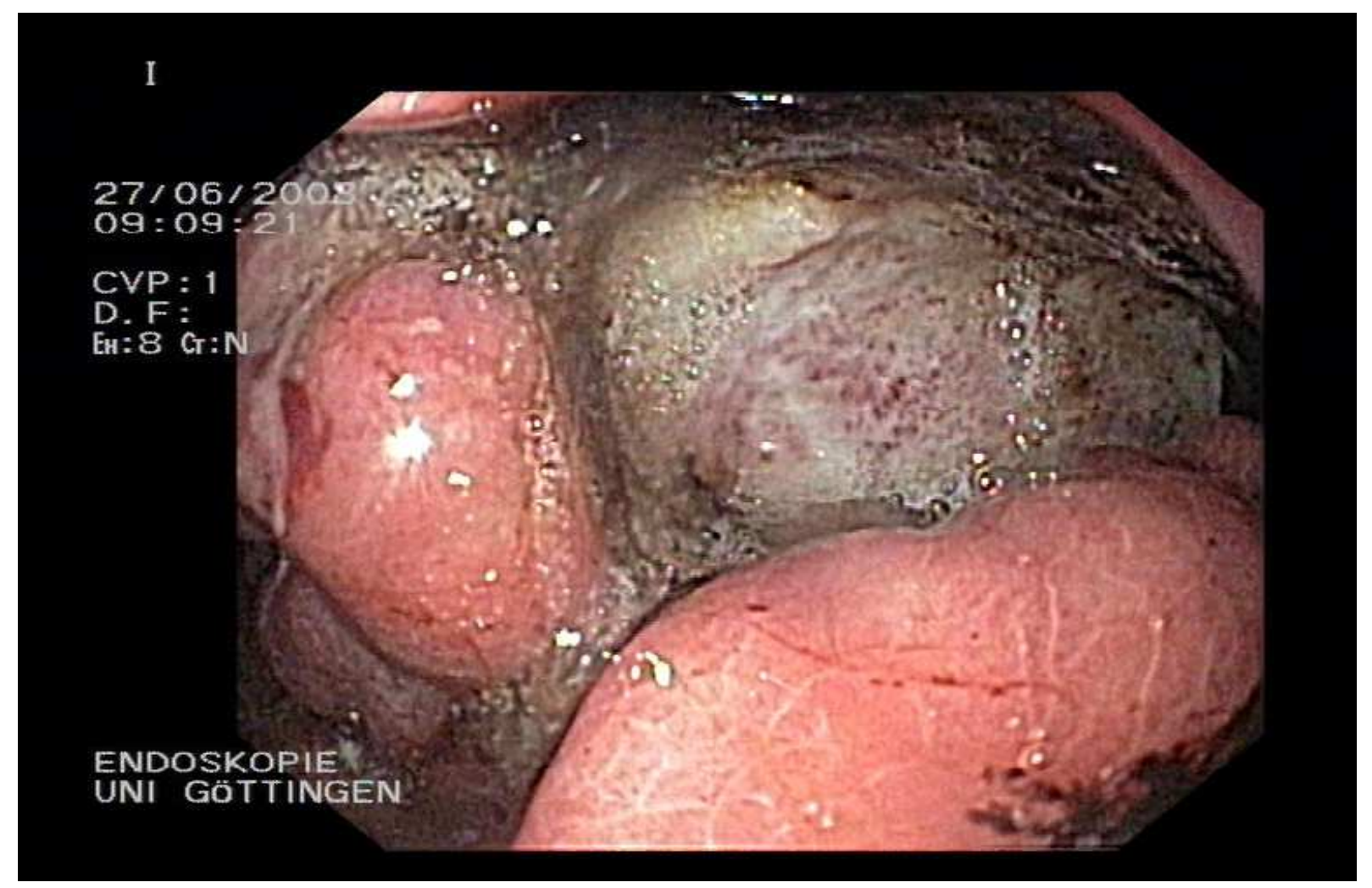

Bild 1: (Patient 8, männlich, 65 Jahre) Ösophagogastroduodenoskopie bei Erstdiagnose zeigt großen Schleimhautdefekt subkardial mit ausgeprägter Ulkushöhle und Fibrinbelägen 


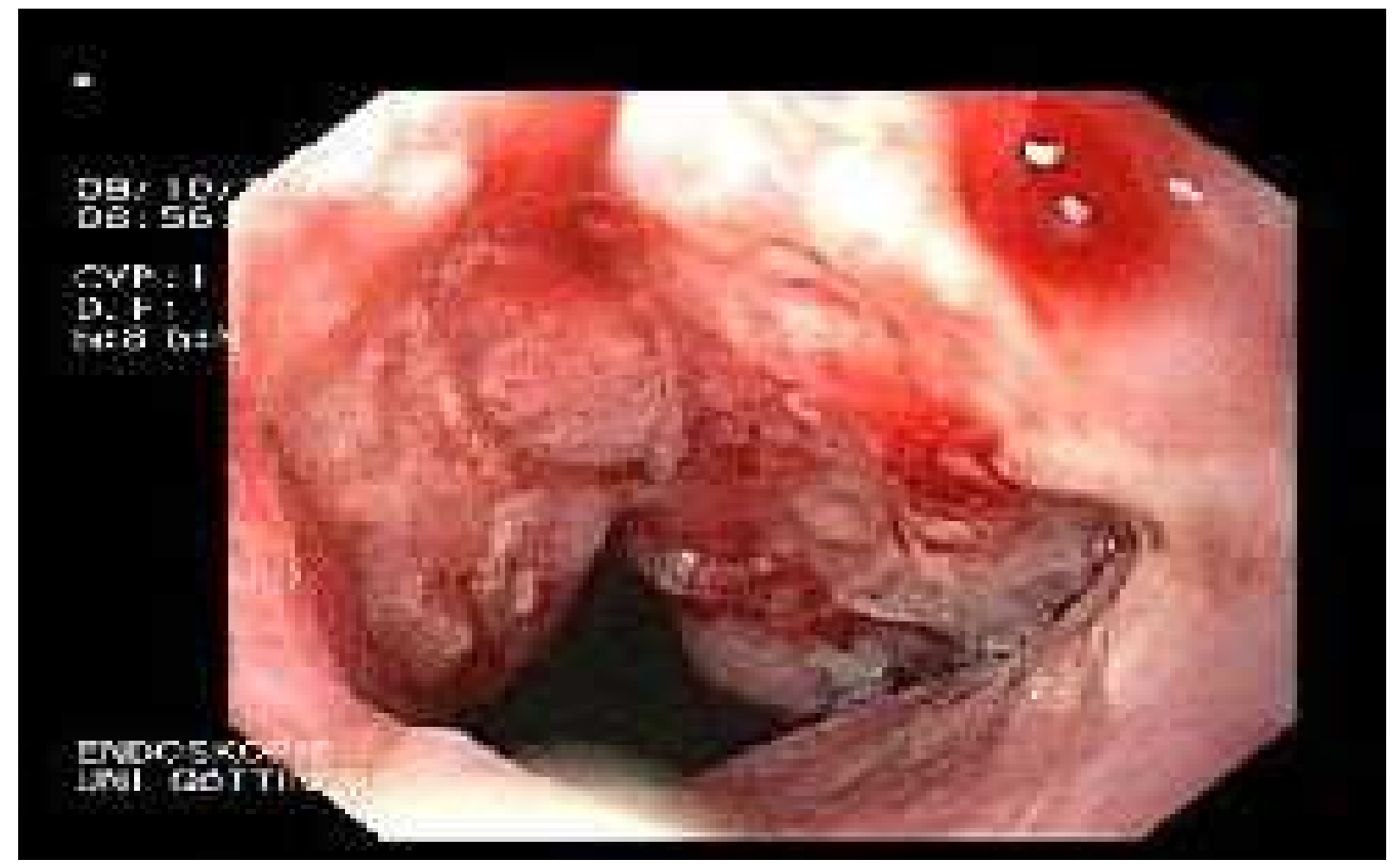

Bild 2: (Patient 16, männlich, 61 Jahre) Ösophago-Gastro-Duodenoskopie bei Erstdiagnose zeigt passierbares, exophytisch und ulzerierend wachsendes Karzinom im Bereich der Kardia

Nach direkter Fixierung der Biopsien in Formalin erfolgte unmittelbar die Übersendung an das Zentrum für Pathologie des Universitätsklinikums Göttingen (unter Leitung von Prof. Dr. med. L. Füzesi), wo nach Eingang der Gewebeproben innerhalb von 5 bis 24 Stunden eine histologische Begutachtung und schriftliche Befundung erfolgte.

Zur Beurteilung der Magenwandarchitketur und der lokalen Lymphknoten schloss sich in 16 Fällen an die ÖGD eine endoluminale Ultraschalluntersuchung (EUS) an.

Ein Patient hat nach der Aufklärung eine EUS abgelehnt. Zur Anwendung kam das Endosonongraphiegerät EUB 7000 HV der Marke Hitachi Medical Systems GmbH (Wiesbaden, Deutschland) mit radialen 5-10 MHz Ultraschallköpfen der Marke Pentax Europe GmbH (Hamburg, Deutschland). Bei nüchternem Zustand wurden die Patienten mit $5 \mathrm{mg}$ Dormicum (Midazolam) sediert. 


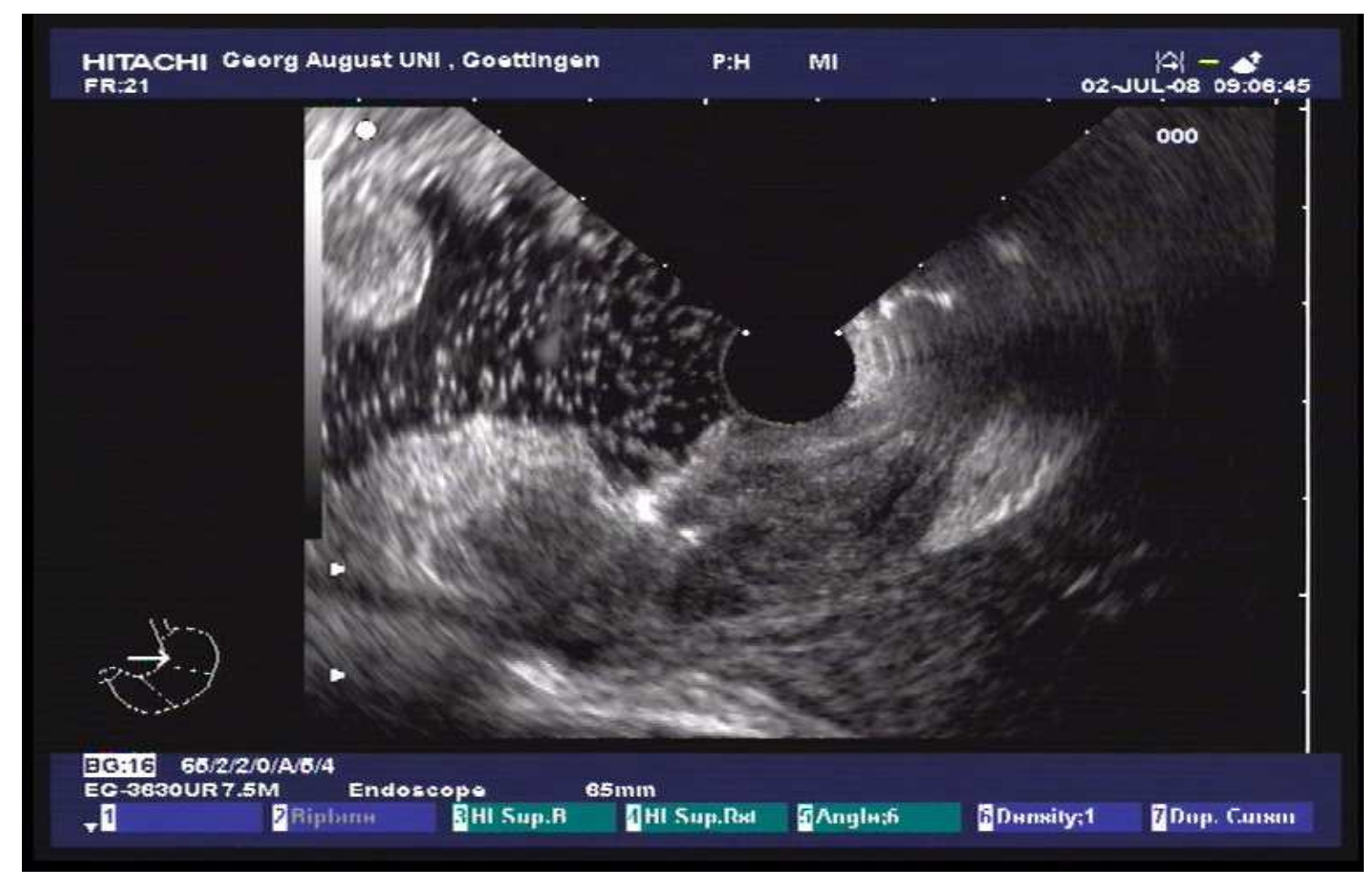

Bild 3: (Patient 8, männlich, 65 Jahre) Endosonographie bei Erstdiagnose zeigt Aufhebung der Wandschichtung kleinkurvaturseitig und zoeliakal sowie paragastral multiple echoarme Lymphknoten

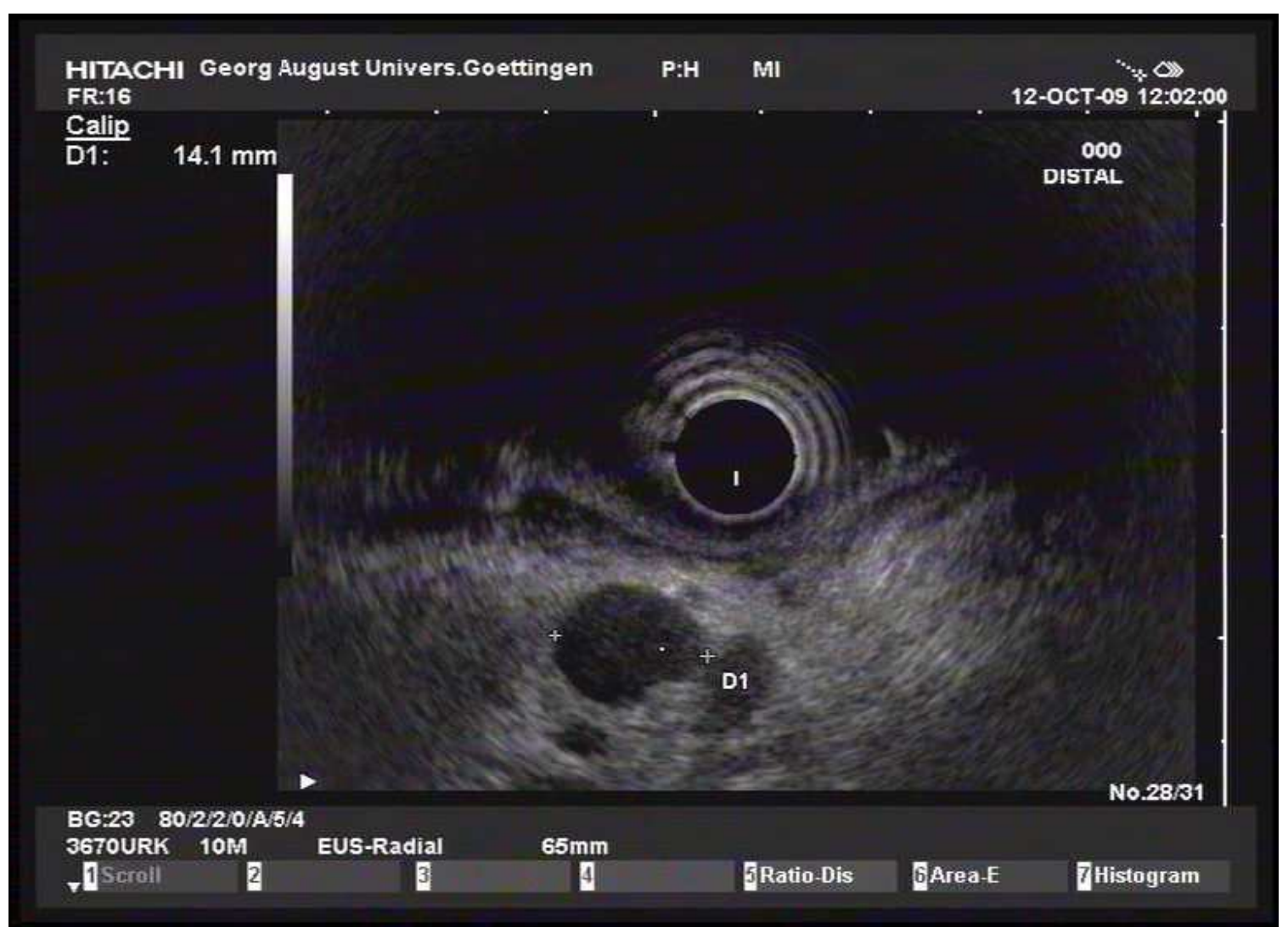

Bild 4: (Patient 16 , männlich, 61 Jahre) Endosonographie bei Erstdiagnose zeigt Kardiakarzinom mit Überschreitung der Muscularis propria 
Als begleitende prätherapeutische Stagingmaßnahmen wurde bei allen 17 Patienten eine Abdomensonographie durchgeführt, wobei das Ultraschall-Gerät EUB 5000 Plus G der Marke Hitachi Medical Systems GmbH (Wiesbaden, Deutschland) zum Einsatz kam. Bei 16 Patienten schloss sich eine computertomographische Untersuchung an. Bei einem Patienten wurde aufgrund einer manifesten Jodallergie eine Magnetresonanztomographie der Computertomographie vorgezogen. Für eine Präzisierung der Ausbreitungsdiagnostik wurde bei 3 Patienten mit Verdacht auf peritoneale Metastasierung eine erweiterte diagnostische Laparoskopie (EDL) angeschlossen. Zur Anwendung kam dabei ein Laparoskop der Marke Richard Wolf GmBH (Knittlingen, Deutschland) mit Durchmessern von 5 bis $10 \mathrm{~mm}$ und einem optischen Winkel von $30^{\circ}$.

Vier Patienten wurden nach der Erstdiagnose zusätzlich auf eine Amplifikation des HER2-Gens (c-erbB-2) auf DNA-Ebene untersucht. Grundlage dieser erweiterten Diagnostik sind die vielversprechenden Ergebnisse der ToGA-Studie, einer international angelegten Phase-III-Studie unter Leitung von Eric van Cutsem (Universitätsklinik Gasthuisberg/ Löwen, Belgien), die auf der 45. Jahresvollversammlung der "American Society of Clinical Oncology (ASCO)“ im Mai/Juni 2009 präsentiert wurden und denen zufolge eine kombinierte Gabe von Herceptin ${ }^{\circledR}$ (Trastuzumab) und Standardchemotherapeutika eine signifikante Verlängerung des Überlebens von Patienten mit HER2-überexprimierendem Magenkarzinom bewirkt (Van Cutsem et al. 2009). Bei HER2 (human epidermal growth factor receptor 2) handelt es sich um einen membranständigen epidermalen Wachstumsfaktor-Rezeptor, der in seiner Funktion die Zellproliferation über den RAS/MAP-Kinase-Weg stimuliert und gleichzeitig die Apoptose durch Beeinflussung der mTor-Signaltransduktion hemmt (Karamouzis et al. 2007). Ursächlich für eine Überexpression des HER2 ist eine Amplifikation des c-erbB2-Gens im Zellkern der Tumorzelle, wie sie bei $22 \%$ aller Patienten mit fortgeschrittenem Magenkarzinom vorkommt (Bang et al. 2008). Herceptin ${ }^{\circledR}$ (Trastuzumab) ist ein monoklonaler Antikörper der HER2 gezielt außer Funktion setzt und damit eine Hemmung der Tumorzellproliferation bewirkt (Olayioye 2001). Der Einsatz von Herceptin® als Tumortherapeutikum war bislang nur aus der Brustkrebstherapie bekannt. Ähnlich wie beim Magenkrazinom überexprimieren 20-25\% aller Mammakarzinompatientinnen HER2 (Untch et al. 2006). Bereits seit dem Jahr 2000 findet Herceptin® in der 
Therapie des metastasierenden HER2-positiven Mammakarzinoms Anwendung. Im Jahr 2006 folgte die Zulassung für Brustkrebs im Frühstadium.

\subsubsection{Perioperative Chemotherapieschemata}

Die Chemotherapie wurde prä- und postoperativ in dem Therapieverlauf und der Patientenkonstitution angepassten Schemata verabreicht. Dabei wurde wahlweise auf eine Kombination aus Epirubicin, Oxaliplatin und Xeloda ${ }^{(}$(Wirkstoff Capecitabine) ( = EOX-Schema), beziehungsweise aus Epirubicin, Oxaliplatin und 5-Fluorouracil ( = EOF-Schema) zurückgegriffen. Im Falle eines Patienten wurde eine Kombination aus 5-Fluorouracil, Folinsäure, Oxaliplatin und Docetaxel eingesetzt (= FLOT-Schema). Die Entscheidung, ob neben Epirubicin und Oxaliplatin Xeloda® oder 5-Fluorouracil verabreicht wurde, war dabei abhängig von der Patintenkonstitution. Bei erhaltener Darmpassage konnte Xeloda $\AA$ in Tablettenform verabreicht werden, während bei tumor- oder operationsabhängigen Komplikationen bei der Nahrungsaufnahme auf 5Fluorouracil kombiniert mit einer mobilen Infusionspumpe zurückgegriffen wurde, was bei 24-stündiger Dauerinfusion mit deutlichen Einschränkungen für den Patienten verbunden ist. Bei einem Patienten wurde wegen des hochgradigen Verdachtes auf ein metastatisches Geschehen im Bereich des Lungenoberfeldes im letzten adjuvanten Chemotherapiezyklus neben einem EOX-Schema zusätzlich der Angiogenese-Inhibitor Avastin ${ }^{\circledR}$ (Wirkstoff Bevacizumab) verabreicht. Durch seine Zugehörigkeit zur Gruppe der Immunglobuline ist dieser Wirkstoff nicht zum eigentlichen Chemotherapieschema zu zählen, sei aber an dieser Stelle der Vollständigkeit halber trotzdem erwähnt.

Die Dosierung der Chemotherapeutika erfolgte in Abhängigkeit von der Körperoberfläche des Patienten in Quadratmetern (KOF) nach folgendem Schema:

\footnotetext{
Epirubicin $50 \mathrm{mg} / \mathrm{m}^{2}$

Oxaliplatin $130 \mathrm{mg} / \mathrm{m}^{2}$

5-Fluoruracil $200 \mathrm{mg} / \mathrm{m}^{2}$

Xeloda $®$ (Capecitabine) $625 \mathrm{mg} / \mathrm{m}^{2}$

Docetaxel $50 \mathrm{mg} / \mathrm{m}^{2}$

Folinsäure $200 \mathrm{mg} / \mathrm{m}^{2}$.
} 
Eine Übersicht über Verteilung und Kombination der Therapieschemata innerhalb des Kollektivs gibt Tabelle 4.

\begin{tabular}{|c|c|c|}
\hline \multirow{2}{*}{$\begin{array}{c}\text { Fallzahl im } \\
\text { Kollektiv }\end{array}$} & \multicolumn{2}{|c|}{ Perioperative Chemotherapie } \\
\cline { 2 - 3 } & $\begin{array}{c}\text { präoperatives } \\
\text { Schema }\end{array}$ & postoperatives \\
Schema
\end{tabular}

Tab. 4: perioperative chemotherapeutische Schemata im Patientenkollektiv; EOX = Epirubicin, Oxaliplatin, Xeloda® / EOF = Epirubicin, Oxaliplatin, 5-Fluoruracil / FLOT = 5-Fluorouracil, Folinsäure, Oxaliplatin und Docetaxel / $d=$ Tage

* bedingt durch reduzierten Allgemeinzustand 


\subsubsection{Präoperatives Staging}

Im Zeitraum zwischen dem letzten neaodjuvanten Chemotherapiezyklus und der Operation wurde bei allen Patienten ein erneutes Staging zur Begutachtung der Tumorgröße und der lokalen Lymphknoten mit Hilfe von ÖGD ,EUS und CT durchgeführt.

Ablauf und Gerät entsprachen der Erstdiagnostik ( $\rightarrow$ 7.2.1).

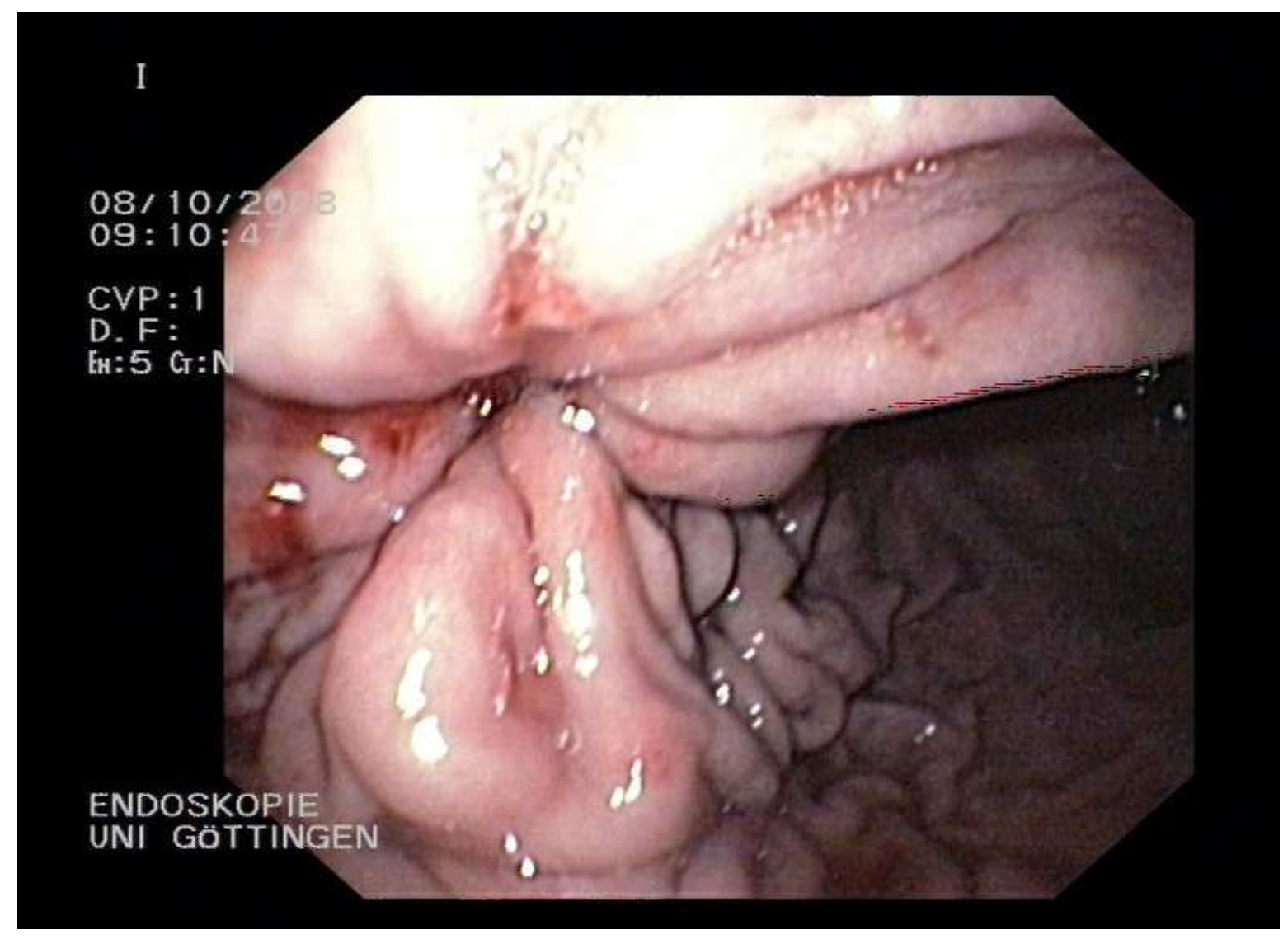

Bild 5: (Patient 8, männlich, 65 Jahre) ÖGD präoperativ nach 3 Zyklen Chemotherapie nach EOXSchema zeigt deutliche Befundbesserung des vorbeschriebenen Tumors subkardial 


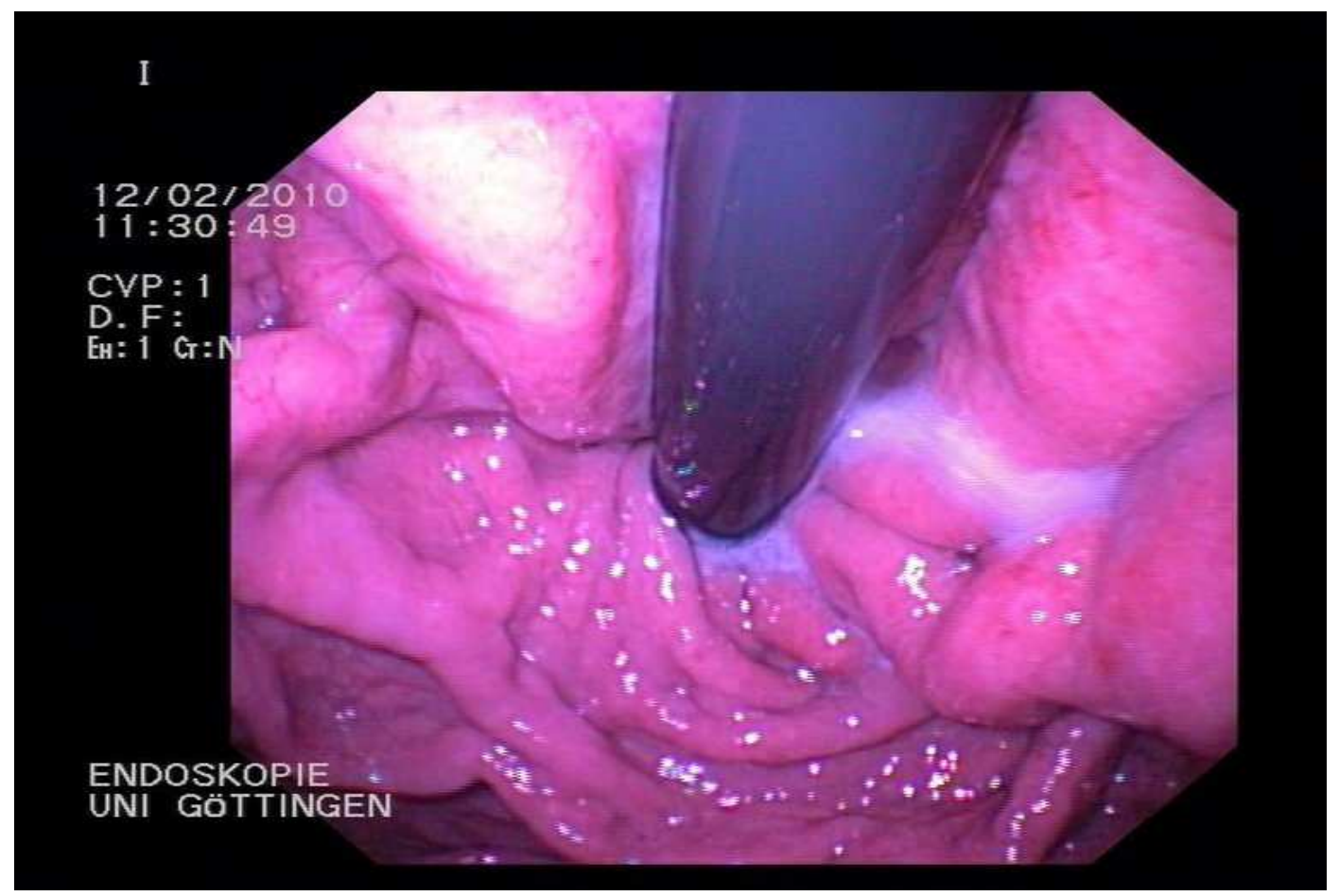

Bild 6: (Patient 16, männlich, 61 Jahre) ÖGD präoperativ nach 3 Zyklen Chemotherapie nach FLOTSchema zeigt deutliche Befundbesserung des vorbeschriebenen kardialen Tumors unter präoperativer Therapie

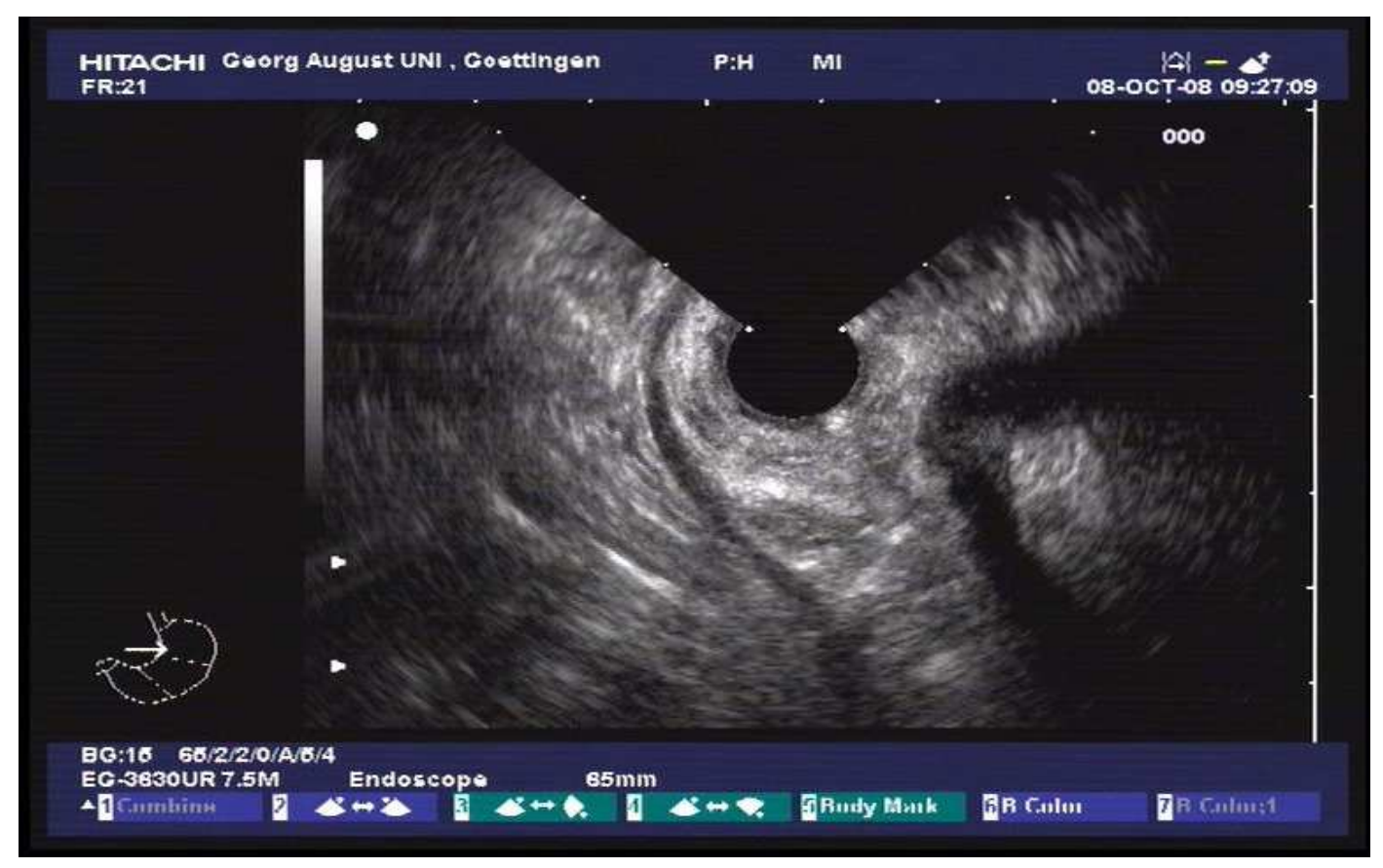

Bild 7: (Patient 8, männlich, 65 Jahre) Endosonographie präoperativ nach 3 Zyklen Chemotherapie nach EOX-Schema zeigt deutliche Rückbildung des vorbeschriebenen Tumors subkardial / Lymphknotenvergrößerung nicht mehr nachweisbar 


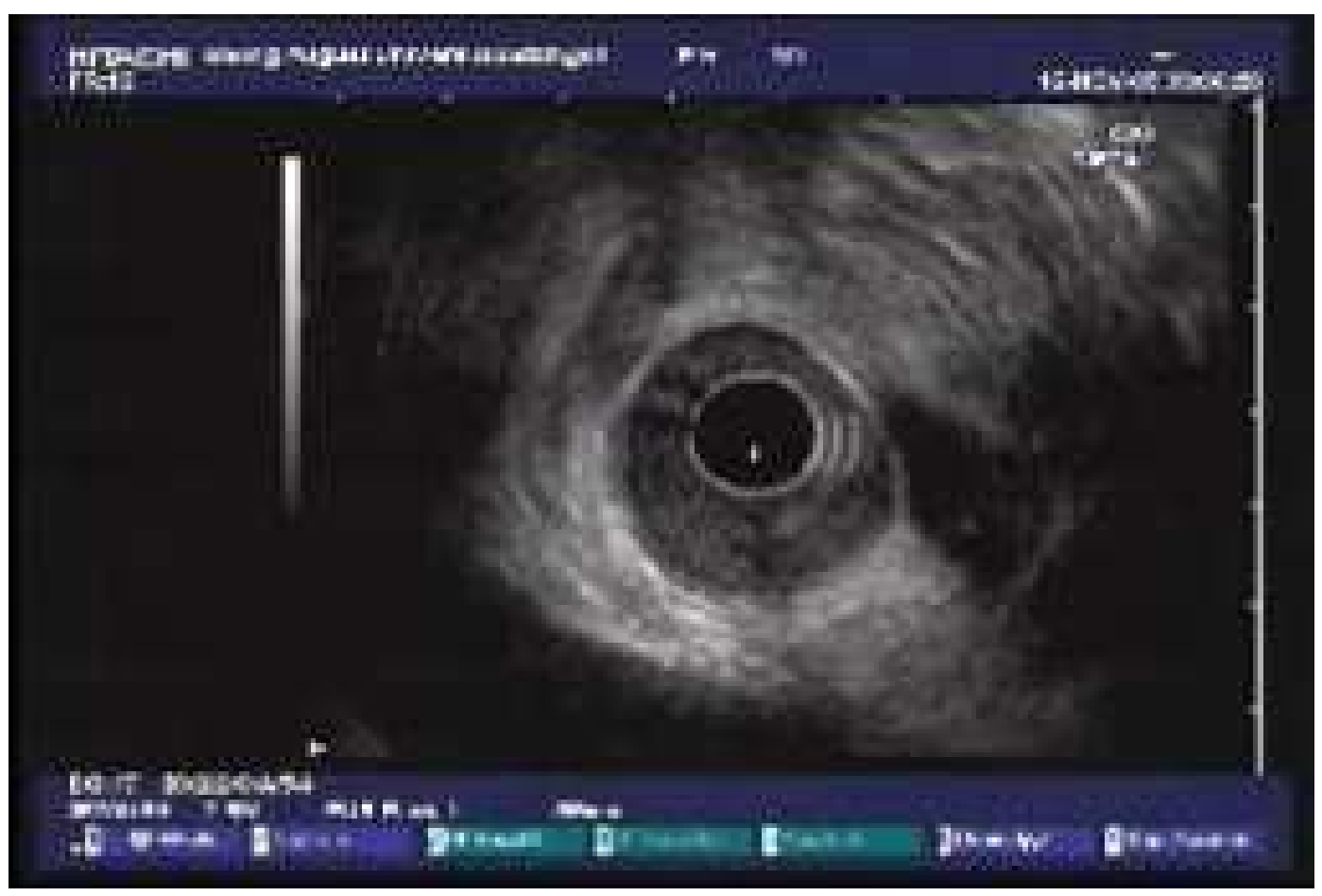

Bild 8 : (Patient 16, männlich, 61 Jahre) Endosonographie präoperativ im Anschluss an 3 Zyklen Chemotherapie nach FLOT-Schema zeigt keine Wandüberschreitung oder Wandinfiltration des vorbeschriebenen Tumors

\subsubsection{Chirurgische Therapie}

Bei allen Patienten wurde unter kurativem Gesichtspunkt mit dem Bestreben einer Resektion im gesunden Gewebe operiert. Bei 7 Patienten mit distaler Tumorlokalisation erfolgte eine totale Gastrektomie, bei 9 Patienten mit Tumorlokalisation im proximalen Magendrittel wurde eine transhiatal erweiterte Gastrektomie durchgeführt. Ein Patient wurde dabei aufgrund eines großflächigen Tumoreinbruchs in Mesocolon transversum und Pankreasschwanz einer multiviszeralen Resektion unterzogen, bei der neben einer totalen Gastrektomie eine Splenektomie mit Pankreaslinksresektion sowie eine Dissektion des Colon transversum durchgeführt wurden. In diesem Fall sowie in zwei weiteren (3 Fälle) konnte lediglich eine R1-Situation erzielt werden, während in den restlichen 13 Fällen der Tumor in sano entfernt wurde.

In einem Fall des Kollektivs zeigte sich intraoperativ eine Peritonealkarzinose sowie eine Metastasierung per continuitatem des Omentum majus, deren Ausmaß die Operateure veranlasste, den Eingriff ohne Gastrektomie abzubrechen. Dieser Patient 
wird im Ergebnisteil lediglich bei „8.1 Alter und Geschlecht“, „8.2 Tumorlokalisation“, „8.3 Tumorstadien innerhalb des Kollektivs vor und nach präoperativer Chemotherapie“ sowie „8.4 Tumorremissionsrate“ berücksichtigt.

\subsubsection{Histologische Befundung des Gastrektomiepräparats}

Eine erste histologisch-pathologische Befundung des Gastrektomiepräparats erfolgte bereits intraoperativ mittels Schnellschnittuntersuchung durch das Zentrum für Pathologie des Universitätsklinikums Göttingen. Das Ergebnis wurde dem Operateur noch während des Eingriffs telefonisch übermittelt.

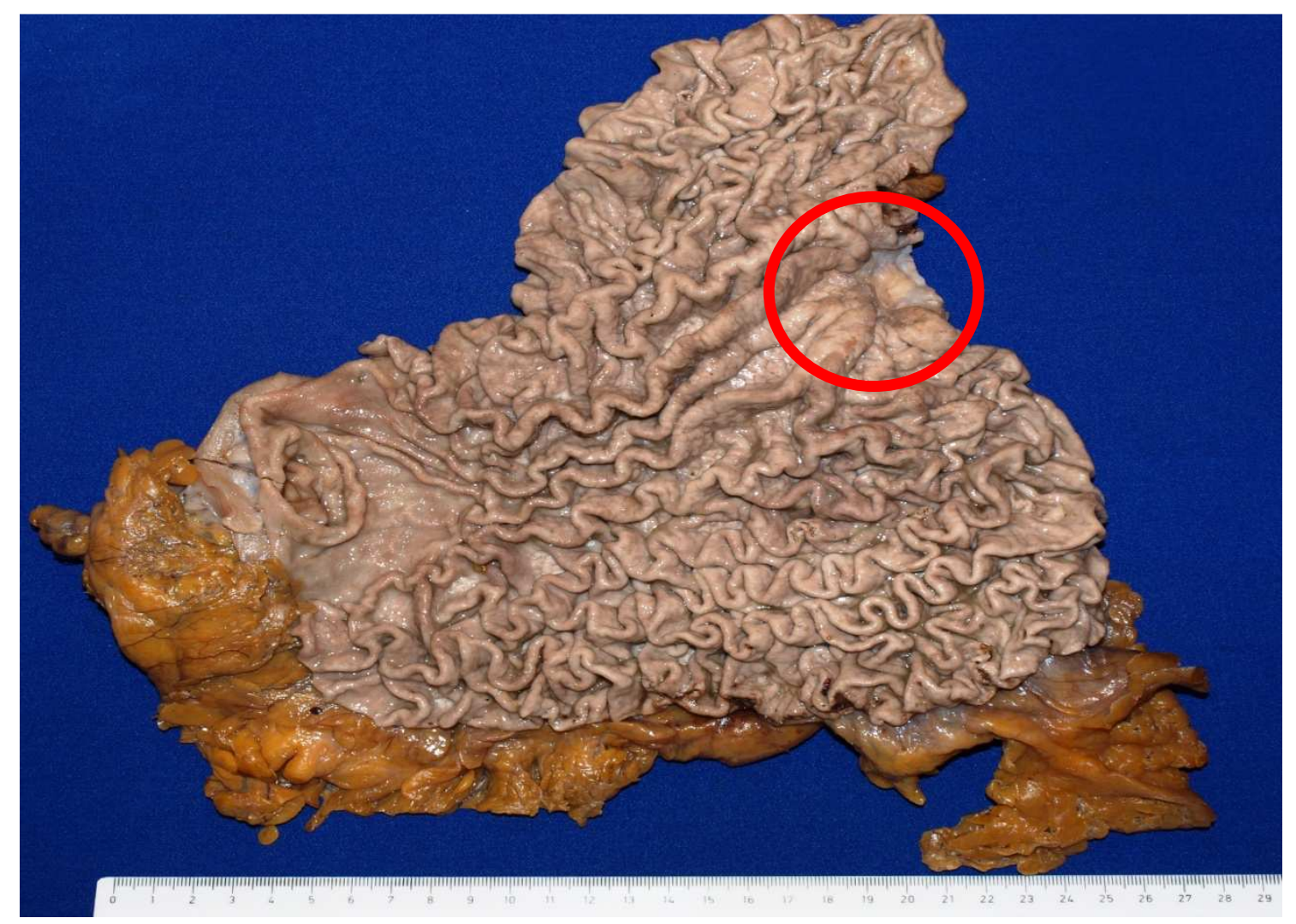

Bild 9 : (Patient 16, männlich, 61 Jahre) Gastrektomiepräparat / Tumorareal mit Primärtumor nach 3 Zyklen Chemotherapie im FLOT-Schema rot umkreist

Anschließend erfolgte am formalinfixierten, paraffineingebetteten OP-Präparat eine umfassende histologische Untersuchung, bei der neben der Bestimmung des vorliegenden TNM-Stadiums nach UICC (2002) auch eine genaue Bestimmung der 
R-Kategorie, des Differenzierungsgrades des Tumorgewebes sowie des Tumorregressionsgrades nach Becker erfolgte.

\subsection{Datenerfassung}

Die dieser Studie zugrunde gelegten Daten wurden retrospektiv anhand der Patientenakten gewonnen. Bei den bildgebenden Verfahren Ösophagogastroduodenoskopie (ÖGD), endoskopische Ultraschalluntersuchung (EUS) und erweiterte diagnostische Laparoskopie (EDL) wurden neben der schriftlichen Befundung durch den Untersucher archivierte Filmsequenzen in die Datenerfassung einbezogen.

Die statistische Auswertung wurde anhand folgender Dokumente durchgeführt:

ÖGD- , EUS- und EDL- Filmsequenzen und Befundberichte vor und nach neoadjuvanter Chemotherapie, Abdomensonographie und CT-Befundberichte vor und nach neoadjuvanter Chemotherapie, Befundberichte der histopathologischen Untersuchung der ÖGD-Biopsien bei Erstdiagnose und des Gastrektomiepräparates sowie mit Hilfe des Operationsberichtes.

Die tabellarische und grafische Dokumentation der gesammelten Daten erfolgte unter Anwendung von Microsoft Excel® 2007.

\section{Ergebnisse}

\subsection{Alter und Geschlecht}

Da sich das Patientenalter zum Zeitpunkt des Auftretens erster Symptome anhand der Patientenakten nicht exakt ermitteln lässt, ist im Folgenden das Lebensalter der Patienten zum Zeitpunkt der Erstdiagnose zugrundegelegt. 
Das durchschnittliche Lebensalter des Gesamtkollektivs betrug 62 Jahre ( $\pm 10,75)$, mit einem Altersmaximum von 78 Jahren und einem Altersminimum von 40 Jahren. Die männlichen Patienten waren mit einem mittleren Alter von 66,3 Jahren (maximal 78 , minimal $59, \pm 6,4)$ im Durchschnitt älter als die weiblichen Patienten mit einem mittleren Alter von 57,1 Jahren (maximal 71, minimal 40, \pm 12,89).

Von den 17 neoadjuvant therapierten Patienten waren 8 weiblich $(=47,1 \%)$ und 9 männlich $(=52,9 \%)$. Eine Übersicht über die Altersverteilung innerhalb des Kollektivs gibt Tabelle 5.

\begin{tabular}{|c|c|c|c|c|c|}
\hline & \multicolumn{4}{|c|}{ Patientenalter von ... bis unter ... Jahren } & \multirow[b]{2}{*}{ gesamt } \\
\hline & $40-50$ & $50-60$ & $60-70$ & $70-80$ & \\
\hline weiblich & 3 & 1 & 3 & 1 & 8 \\
\hline männlich & 0 & 1 & 5 & 3 & 9 \\
\hline gesamt & 3 & 2 & 8 & 4 & 17 \\
\hline
\end{tabular}

Tab. 5: Altersverteilung innerhalb des Patientenkollektivs insgesamt und nach Geschlecht aufgeführt

\subsection{Tumorlokalisation}

Im gesamten Kollektiv konnten bei 17 Patienten vor Therapiebeginn 19 tumoröse Läsionen der Magenwand nach ihrer makroskopischen Lokalisation unterschieden werden. Bei 15 Patienten wurde mittels Gastroskopie 1 Primärtumor diagnostiziert, während im Rahmen der Erstdiagnostik bei 2 Patienten des Kollektivs jeweils 2 makroskopische Tumorläsionen festgestellt wurden. 
Dabei handelte es sich in 42,1\% (8 Läsionen) der Fälle um ein Kardiakarzinom (4 x AEG II und 4 x AEG III nach Siewert), in 21,1\% (4 Läsionen) um ein Karzinom des Korpus, in 26,3\% (5 Läsionen) wurde ein Karzinom des Antrums diagnostiziert. Bei 10,5\% (2 Läsionen) dehnte sich der Primärtumor als Linitis plastica über mehrere Anteile des Magens aus. Eine Übersicht über die Tumorlokalisation innerhalb des Kollektivs gibt Abbildung 4.

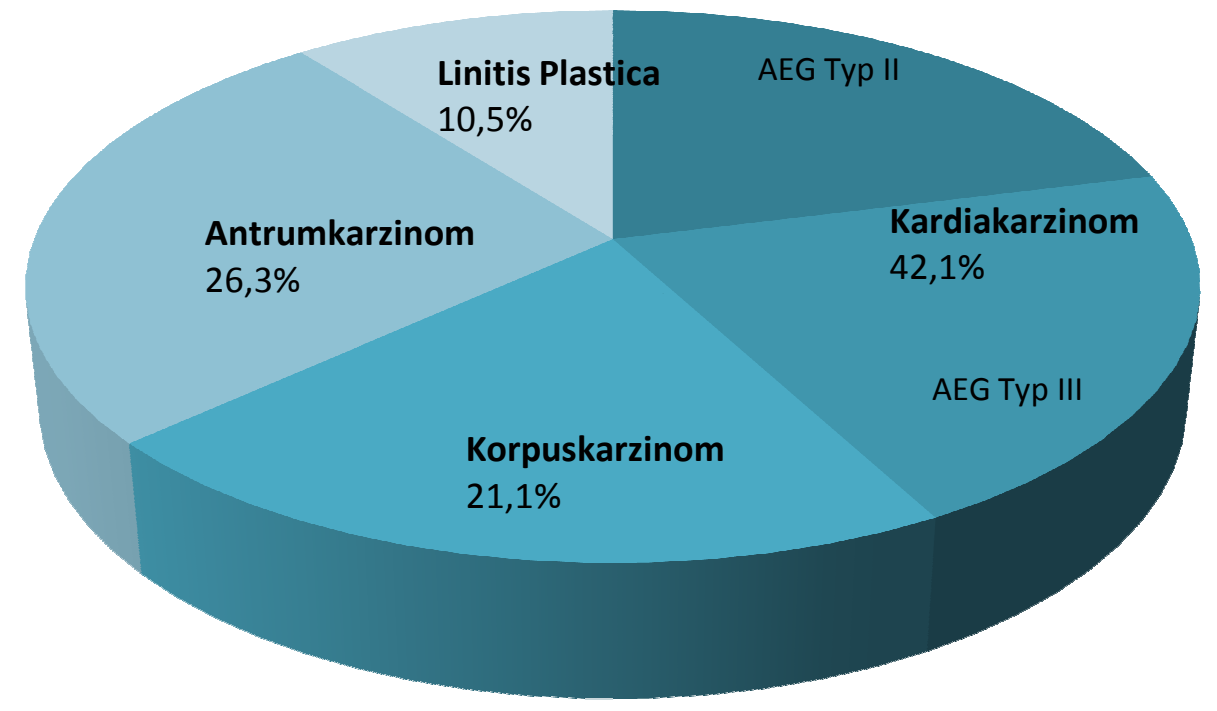

Abb.4 : Tumorlokalisation innerhalb des Patientenkollektivs

8.3 Tumorstadien innerhalb des Kollektivs vor und nach präoperativer Chemotherapie

\subsubsection{Tumorstadien vor präoperativer Chemotherapie (nach UICC, 2002)}

Gemäß den Richtlinien der UICC zur Bestimmung des Tumorstadiums konnte das Patientenkollektiv anhand des endosonographischen Befundes nach Erstdiagnose wie folgt aufgeteilt werden: In 2 Fällen (11,8\%) des Kollektivs wurde Stadium IB 
diagnostiziert, in 4 Fällen Stadium II (23,5\%), in 10 Fällen Stadium IIIA $(58,8 \%)$ und in einem Fall Stadium IV (5,9\%).

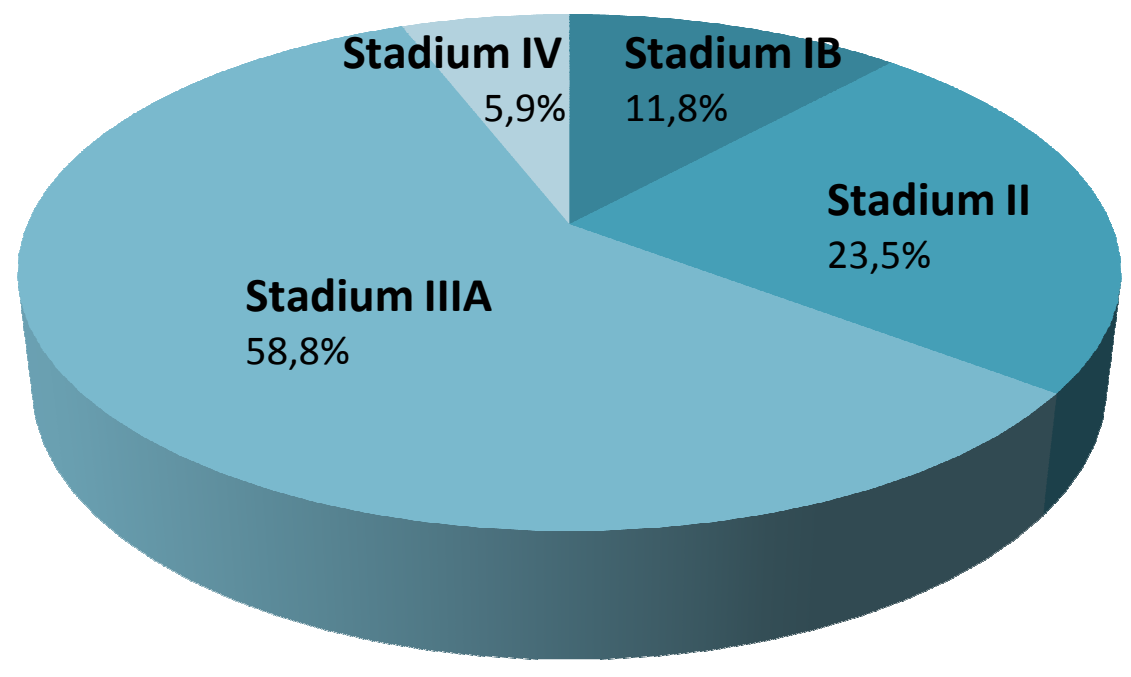

Abb.5 : Stadienverteilung nach UICC (2002) innerhalb des Patientenkollektivs vor präoperativer Chemotherapie / Bestimmung anhand des endosongraphischen Befundes

8.3.2 Tumorstadium nach präoperativer Chemotherapie (nach UICC, 2002)

Gemäß den Richtlinien der UICC zur Bestimmung des Tumorstadiums konnte das Patientenkollektiv anhand des Gastrektomiepräparates wie folgt aufgeteilt werden: In 2 Fällen (11,8\%) wurde Stadium IA diagnostiziert, in 3 Fällen Stadium IB (17,6\%), in 4 Fällen Stadium II (23,5\%), in 3 Fällen Stadium IIIA (17,6\%) und in 5 Fällen Stadium IV $(29,4 \%)$. Der Stadienzuordnung des Patienten ohne Gastrektomie liegt die intraoperativ festgestellte Metastasierung in Nachbarstrukturen zugrunde. 


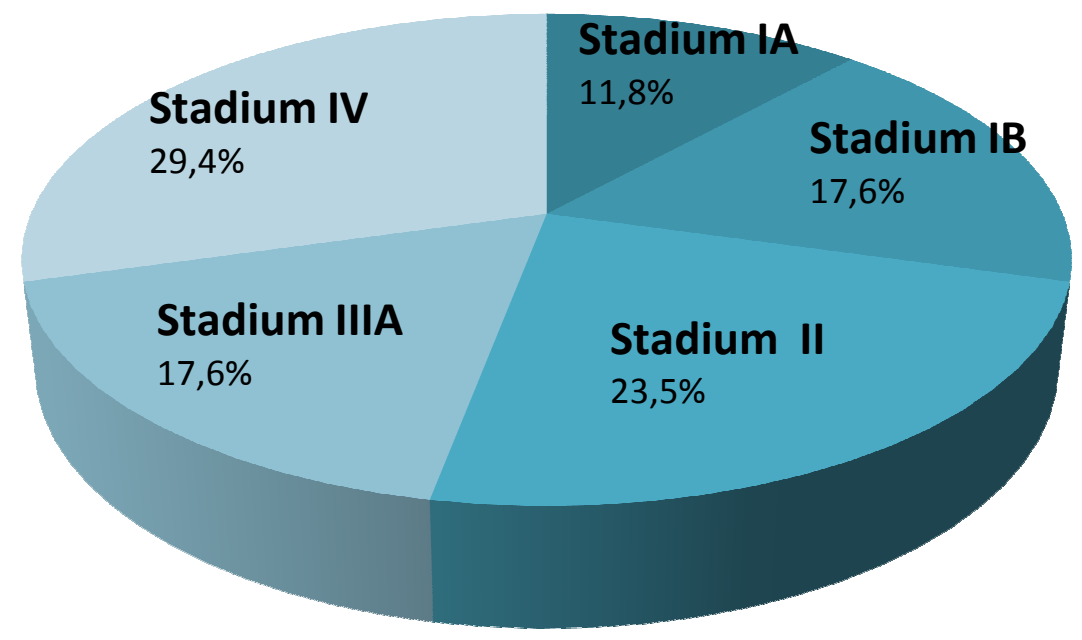

Abb.6 : Stadienverteilung nach UICC (2002) innerhalb des Patientenkollektivs nach präoperativer Chemotherapie / Bestimmung anhand Gastrektomiepräparat

\subsection{Tumorremissionsrate}

Abbildung 7 gibt einen Überblick über den prozentualen Anteil an Patientenfällen des Gesamtkollektivs, bei denen zwischen dem Primärstaging und einem Restaging nach präoperativer Chemotherapie mittels Endosonographie und Ösophagogastroduodenoskopie (ÖGD) ein rückläufiger Größenbefund der Primärläsion erhoben werden konnte.

\section{Tumorremission}

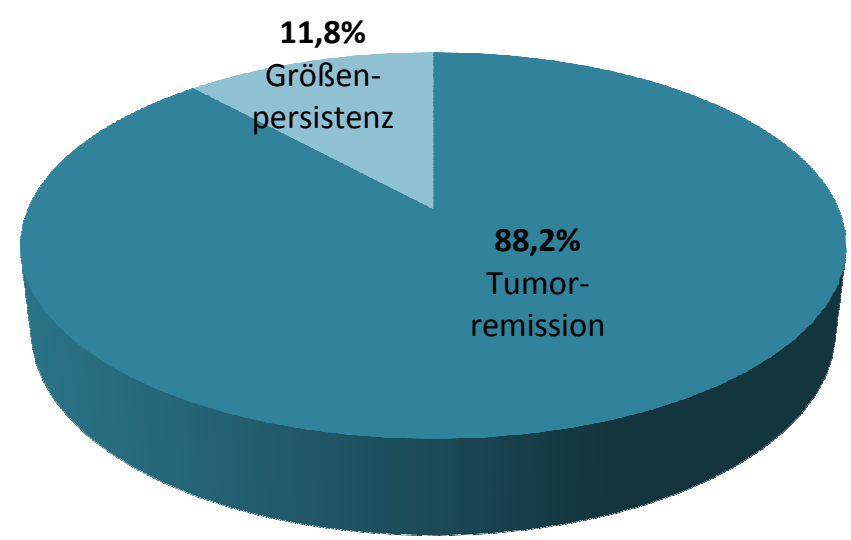

Abb.7 : Tumorremissionsrate des Gesamtkollektivs durch neodajuvante Chemotherapiezyklen 


\subsection{Zeitabstände der perioperativen Chemotherapie}

Abbildung 8 zeigt eine Übersicht über die chronologische Positionierung der neoadjuvanten und adjuvanten Chemotherapie relativ zur Operation. Zugrunde gelegt wurde der Zeitraum zwischen dem letzten / ersten Tag der Chemotherapie und der Operation, wobei die Tage, an denen die Chemotherapie verabreicht wurde, und der Operationstag nicht einbezogen wurden.

Aufgrund früher postoperativer Letalität (38 Tage) wurde Patient 15 in die Ermittlung des Abstandes zwischen Operation und postoperativer Chemotherapie nicht einbezogen. Ebenso Patient 1, bei dem wegen eines stark reduzierten Allgemeinzustandes keine adjuvante Chemotherapie angesetzt werden konnte.

\section{Zeitabstand CTX-OP-CTX (in Tagen)}

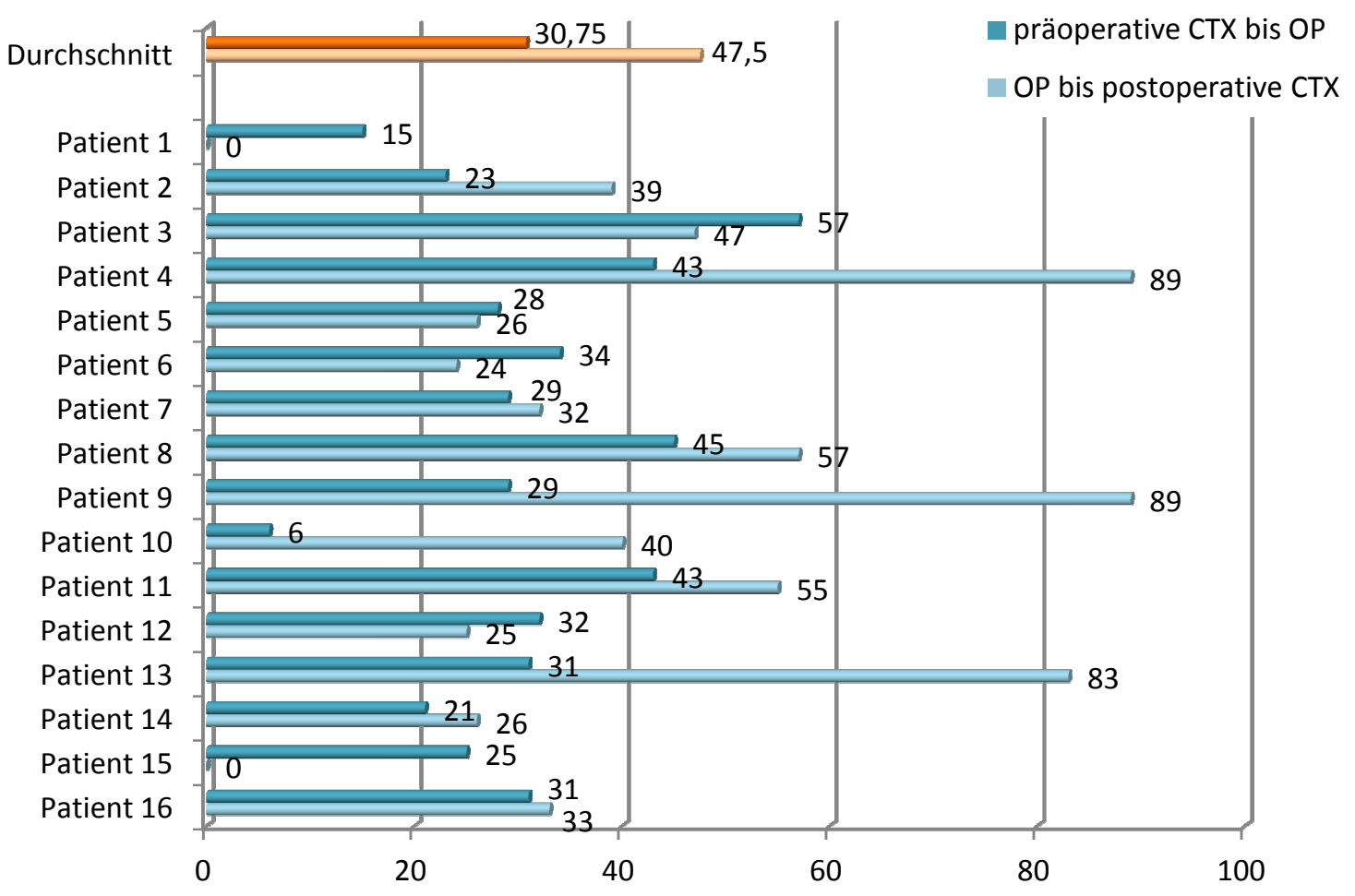

Abb.8 : Zeitabstände zwischen den Therapieschritten neoadjuvante Chemotherapie, Operation und adjuvante Chemotherapie in Tagen / CTX=Chemotherapie/ OP=Operation 
Die durchschnittliche Zeitspanne zwischen dem letzten Zyklus der präoperativen Chemotherapie und der Operation betrug 30,75 Tage (maximal 57, minimal 6, \pm 12,29).

Zwischen Operation und dem ersten Zyklus der adjuvanten Chemotherapie lagen im Durchschnitt 47,5 Tage (maximal 89, minimal 24, $\pm 23,88$ ). Abgesehen von den Patienten (Patient 1, 15), die keine postoperative Chemotherapie erhielten, betrug die minimale Dauer zwischen Operation und erstem Zyklus der adjuvanten Chemotherapie 24 Tage. Im Maximum vergingen 89 Tage.

\subsection{Ausmaß der Lymphadenektomie (LAD)}

\subsubsection{Verteilung von D1-,D2-,D3-Lymphadenektomien}

Bei $87,5 \%$ (14 Fälle) der Patienten des Kollektivs mit Gastrektomie $(n=16)$ konnte eine D2-Lymphadenektomie durchgeführt werden. In einem Fall (6,3\%) beschränkte sich die Resektion auf die perigastralen Lymphknoten entlang der Curvatura major und minor, entsprechend einer D1-Resektion. Dabei sollte Erwähnung finden, dass laut Operationsbericht eine Einbeziehung der Lymphknoten des 2.Kompartiments vor dem Hintergrund einer ausgeprägten Peritonealkarzinose des Patienten als nicht sinnvoll erachtet wurde. In einem weiteren Fall $(6,3 \%)$ erfolgte die systematische Lymphadenektomie im Sinne einer D3-Resektion.

Abbildung 9 gibt einen Überblick über die Verteilungen von D1-, D2-, D3Lymphknotenresektionen innerhalb des Patientenkollektivs. 


\section{Ausmaß der Lymphadenektomie (LAD)}

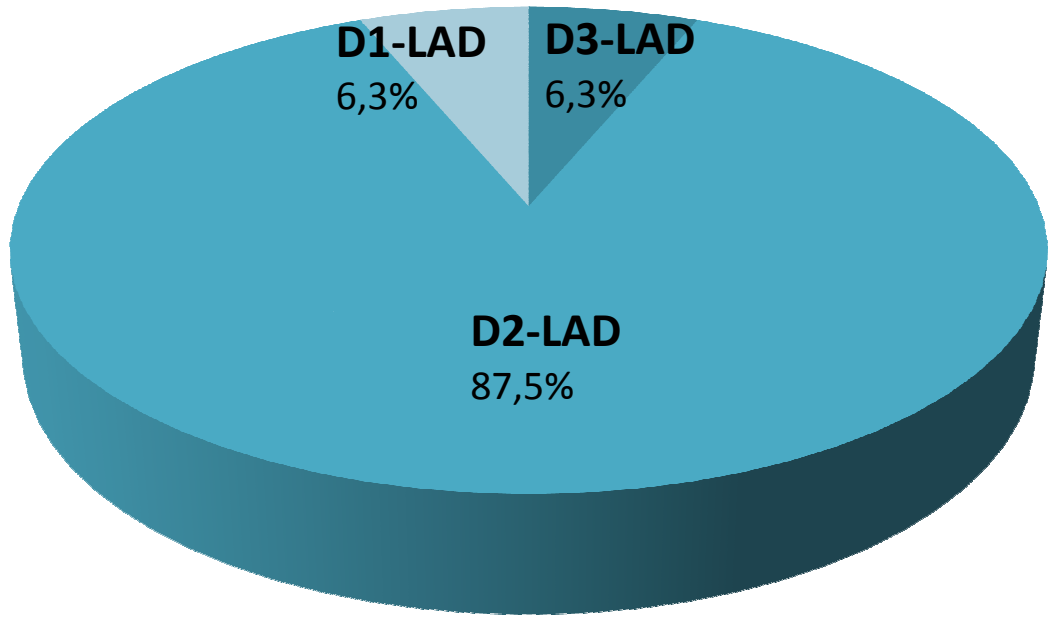

Abb.9: Anteil an D1-, D2-, D3-Lymphadenektomien innerhalb des Patientenkollektivs

8.6.2 Ausmaß der Lymphadenektomie relativ zum UICC-Stadium

\begin{tabular}{|c|c|c|c|c|}
\hline UICC-Stadium & $\begin{array}{l}\text { Fallanzahl im } \\
\text { Kollektiv }\end{array}$ & D1-LAD & D2-LAD & D3-LAD \\
\hline Stadium 0 & $0 \quad(0 \%)$ & 0 & 0 & 0 \\
\hline Stadium la & $2(12,5 \%)$ & 0 & 1 & 1 \\
\hline Stadium Ib & $3 \quad(18,75 \%)$ & 0 & 3 & 0 \\
\hline Stadium II & $4 \quad(25 \%)$ & 0 & 4 & 0 \\
\hline Stadium IIIa & $3 \quad(18,75 \%)$ & 1 & 2 & 0 \\
\hline Stadium IIIb & $0 \quad(0 \%)$ & 0 & 0 & 0 \\
\hline Stadium IV & $4 \quad(25 \%)$ & 0 & 4 & 0 \\
\hline Gesamt & $16(100 \%)$ & 1 & 12 & 1 \\
\hline
\end{tabular}

Tab.6: Ausmaß der Lymphadenektomie im Verhältnis zum UICC-Stadium innerhalb des Kollektivanteils mit Gastektomie 
Tabelle 6 lässt Rückschlüsse auf einen Bezug zwischen Tumor-Stadium (nach UICC, 2002) und Umfang des Lymphknotendissektats zu. Die Bestimmung der Tumorstadien gemäß den Richtlinien der UICC erfolgte dabei wie bei 8.3.2 anhand des Gastrektomiepräparats.

Wie unter 8.6.1 dargestellt, wurde in nur 2 Patientenfällen keine D2-LAD durchgeführt. Deutlich wird, dass sich innerhalb des untersuchten Patientenkollektivs keine direkte Beziehung zwischen Tumorstadium und kompartimentabhängiger Ausdehnung der Lymphknotenresektion feststellen lässt. Während die D1-LAD auf einen Patienten mit fortgeschrittenem Tumorstadium fällt, wurde bei einem Patienten in Stadium la die Resektion auf 3 Kompartimente erweitert. Abgesehen davon, wurden über das gesamte Spektrum der Tumorstadien innerhalb des Kollektivs (Stadium la bis Stadium IV) neben den perigastrischen Lymphknoten auch die Lymphknoten von Truncus coeliacus, Ligamentum hepatoduodenale und Milzhilus in das Gastrektomiepräparat einbezogen. Im Fall des Patienten, bei dem eine D1-LAD einer D2-LAD vorgezogen wurde, bestimmte angesichts der Tatsache einer Peritonealkarzinose und einer Tumorausbreitung bis in distale oesophageale Bereiche vermutlich der palliative Therapiegedanke das weitere chirugische Vorgehen.

\subsection{Entfernte Lymphknoten}

\subsubsection{Anzahl der entfernten Lymphknoten}

Die Angaben zur Anzahl der entfernten und der befallenen Lymphknoten wurden aus dem Befundbericht des Gastrektomiepräparats, erstellt durch das Zentrum für Pathologie der Universitätsmedizin Göttingen, entnommen. Durchschnittlich wurden 29,13 Lymphknoten $( \pm 10,14)$ in das Resektat einbezogen, wobei maximal 56 (Patient 11), minimal 15 Lymphknoten (Patient 3) reseziert wurden. Im gesamten Kollektiv waren zwischen 0 (Patient 4, 8, 9, 11, 15) und 19 (Patient 13) Lymphknoten von metastatischen Absiedlungen befallen, dabei liegt der Mittelwert aller Patienten bei $4,56( \pm 6,22)$ befallenen Lymphknoten im Gastrektomiepräparat. 


\section{Anzahl entfernter und befallener Lymphkn}

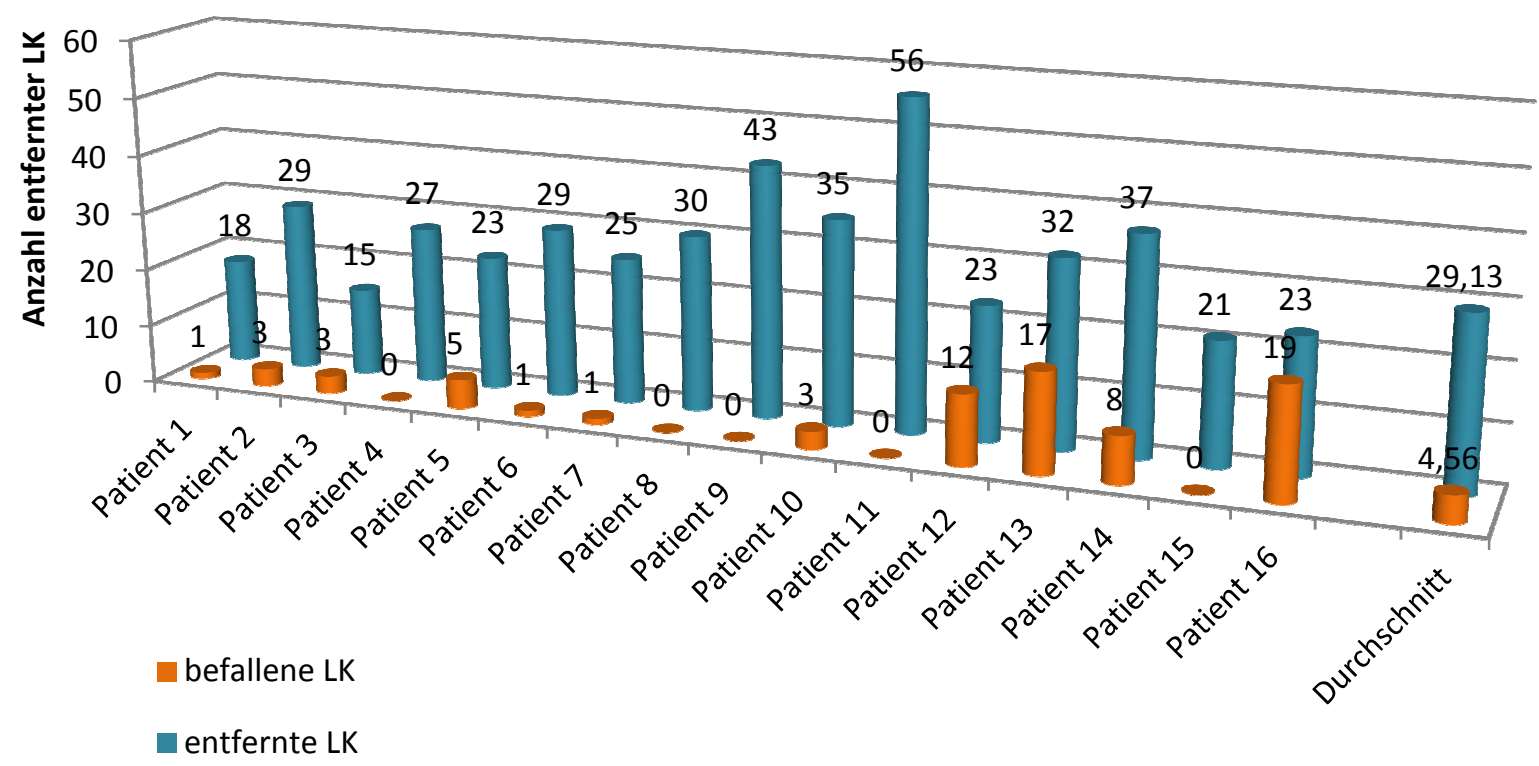

Abb.10: Anzahl der entfernten und befallenen Lymphknoten innehrhalb des Kollektivs und im Durchschnitt

Tabelle 7 zufolge beträgt die durchschnittliche Lymphknotenbefallsrate (lymph node ratio) innerhalb der Patientengruppe 17,69\% (maximal 82,6\%, minimal 0\%, $\pm 24,4$ ).

\subsubsection{Lokalisation befallener Lymphknoten}

Tabelle 7 zeigt die Lokalisation der befallenen Lymphknoten bei allen Patienten des Kollektivs mit totaler und transhiatal erweiterter Gastrektomie (16 Fälle). Während sich in 5 Fällen keine metastatisch besiedelten Lymphknoten im Gastrektomiepräparat feststellen ließen, wiesen 10 Patienten Tumorinfiltrate in Lymphknotenstationen des 1. Kompartiments auf. Lediglich in einem Fall konnten Lymphknoten mit vitalem Tumorzellnachweis sowohl im ersten wie auch im zweiten Kompartiment mit Lokalisation im Milzhilus nachgewiesen werden. 


\begin{tabular}{|c|c|c|c|c|}
\hline Patient & LAD & $\begin{array}{c}\text { entfernte/befallene } \\
\text { Lymphknoten } \\
\text { (LNR) }\end{array}$ & $\begin{array}{l}\text { Lokalisation } \\
\text { befallener LK }\end{array}$ & $\begin{array}{l}\text { befallene LK in } \\
\text { Kompartiment }\end{array}$ \\
\hline Patient 1 & $\mathrm{D} 2$ & $18 / 1$ (5.6\%) & Curvatura minor (1LK) & 1 \\
\hline Patient 2 & D2 & 29 / 3 (10.3\%) & Curvatura minor ( $3 \mathrm{LK}$ ) & 1 \\
\hline Patient 3 & D2 & 15 / 3 (20\%) & Curvatura minor (3 LK) & 1 \\
\hline Patient 4 & D3 & $27 / 0$ (0\%) & - & - \\
\hline Patient 5 & D1 & $23 / 5$ (21.7\%) & $\begin{array}{l}\text { Curvatura major ( } 3 L K \text { ) } \\
\text { Curvatura minor ( } 2 \mathrm{LK} \text { ) }\end{array}$ & 1 \\
\hline Patient 6 & D2 & $29 / 1$ (3.4\%) & Curvatura major (1LK) & 1 \\
\hline Patient 7 & D2 & $25 / 1 \quad(4 \%)$ & Curvatura minor (1LK) & 1 \\
\hline Patient 8 & D2 & $30 / 0(0 \%)$ & - & - \\
\hline Patient 9 & D2 & $43 / 0$ (0\%) & - & - \\
\hline Patient 10 & D2 & $35 / 3(8,6 \%)$ & $\begin{array}{l}\text { Curvatura major (1 LK) } \\
\text { Curvatura minor (2 LK) }\end{array}$ & 1 \\
\hline Patient 11 & $\mathrm{D} 2$ & $56 / 0(0 \%)$ & - & - \\
\hline Patient 12 & $\mathrm{D} 2$ & $23 / 12$ (52.2\%) & $\begin{array}{l}\text { Gastroösophagealer } \\
\text { Übergang (4LK) } \\
\text { Curvatura minor (8LK) }\end{array}$ & 1 \\
\hline Patient 13 & D2 & 32 / 17 (53.1\%) & $\begin{array}{l}\text { Curvatura major ( } 5 \text { LK) } \\
\text { Curvatura minor (11 LK) } \\
\text { Milzhilus (1LK) }\end{array}$ & $1+2$ \\
\hline Patient 14 & $\mathrm{D} 2$ & $37 / 8$ (21.6\%) & Curvatura major (8LK) & 1 \\
\hline Patient 15 & $\mathrm{D} 2$ & $21 / 0(0 \%)$ & - & - \\
\hline Patient 16 & D2 & $23 / 19$ (82.6\%) & $\begin{array}{l}\text { Curvatura major ( } 5 \text { LK) } \\
\text { Curvatura minor (14 LK) }\end{array}$ & 1 \\
\hline- & - & $\varnothing$ LNR $17,69 \%$ & - & - \\
\hline
\end{tabular}

Tab. 7: Lokalisation befallener Lymphknoten (LK) / LAD = Lymphadenektomie / LNR= lymph node ratio 
8.7.3 Präzision in der Beurteilung des Lymphknotenbefalls bei endoskopischer und computertomographischer Diagnostik

Entsprechend der aktuellen Bestimmungen der UICC (2002) erfolgte die Bestimmung der Lymphknotenmetastasierung anhand der Anzahl regionär befallener Lymphknoten mit Zuordnung zu den Stadien N0, N1, N2, N3. Im Folgenden werden die Diagnosen der präoperativen endoskopischen Ultraschalluntersuchung ( $\mathrm{cN}$ (eus)) und der präoperativen computertomographischen Untersuchung $(\mathrm{cN}(\mathrm{ct}))$ mit dem Ergebnis der pathohistologischen Untersuchung am Operationsresektat $(\mathrm{pN})$ als Referenz verglichen.

Aufgrund des Operationsabbruchs und damit fehlendem pN-Stadium eines Patienten wurden die Daten dieses Falles im Folgenden nicht berücksichtigt.

\begin{tabular}{|c|c|c|c|c|}
\hline & N0 & N1 & N2 & N3 \\
\hline $\mathrm{pN}$ & $4(25 \%)$ & $7(43,75 \%)$ & $3(18,75 \%)$ & $2(12,5 \%)$ \\
\hline $\mathrm{cN}($ eus $)$ & $4(25 \%)$ & $9(56,25 \%)$ & $3(18,75 \%)$ & $0(0 \%)$ \\
\hline $\mathrm{cN}$ (ct) & $4(25 \%)$ & $10(62,5 \%)$ & $2(12,5 \%)$ & $0(0 \%)$ \\
\hline
\end{tabular}

Tab.8: Anzahl der diagnostizierten N-Stadien in endosonographischer und computertomographischer Untersuchung sowie im Operationresektat entsprechend UICC-Richtlinien (2002)

Wie Tabelle 8 und Abbildung 11 zeigen, wurden im pathologischen Resektat in 4 Fällen Stadium N0 (25\%), in 7 Fällen Stadium N1(43,75\%), in 3 Fällen Stadium N2 $(18,75 \%)$ sowie in 2 Fällen Stadium N3 (12,5\%) festgestellt.

Bei der endoskopischen Ultraschalluntersuchung wurden innerhalb des Kollektivs ebenfalls in 4 Fällen Stadium N0 (25\%), in 9 Fällen Stadium N1 (56,25\%), in 3 Fällen Stadium N2 (18,75\%) und in keinem der Patientenfälle Stadium N3 diagnostiziert. 
Eine ähnliche Verteilung lässt sich in der computertomographischen Lymphknotendiagnostik feststellen. In 4 Fällen wurde eine N0-Situation diagnostiziert (25\%), in 10 Fällen ein N1-Stadium (62,5\%), in 2 Fällen ein N2-Stadium(12,5\%) sowie in keinem Fall ein N3-Stadium.

\section{N-Stadium entsprechend EUS / CT}

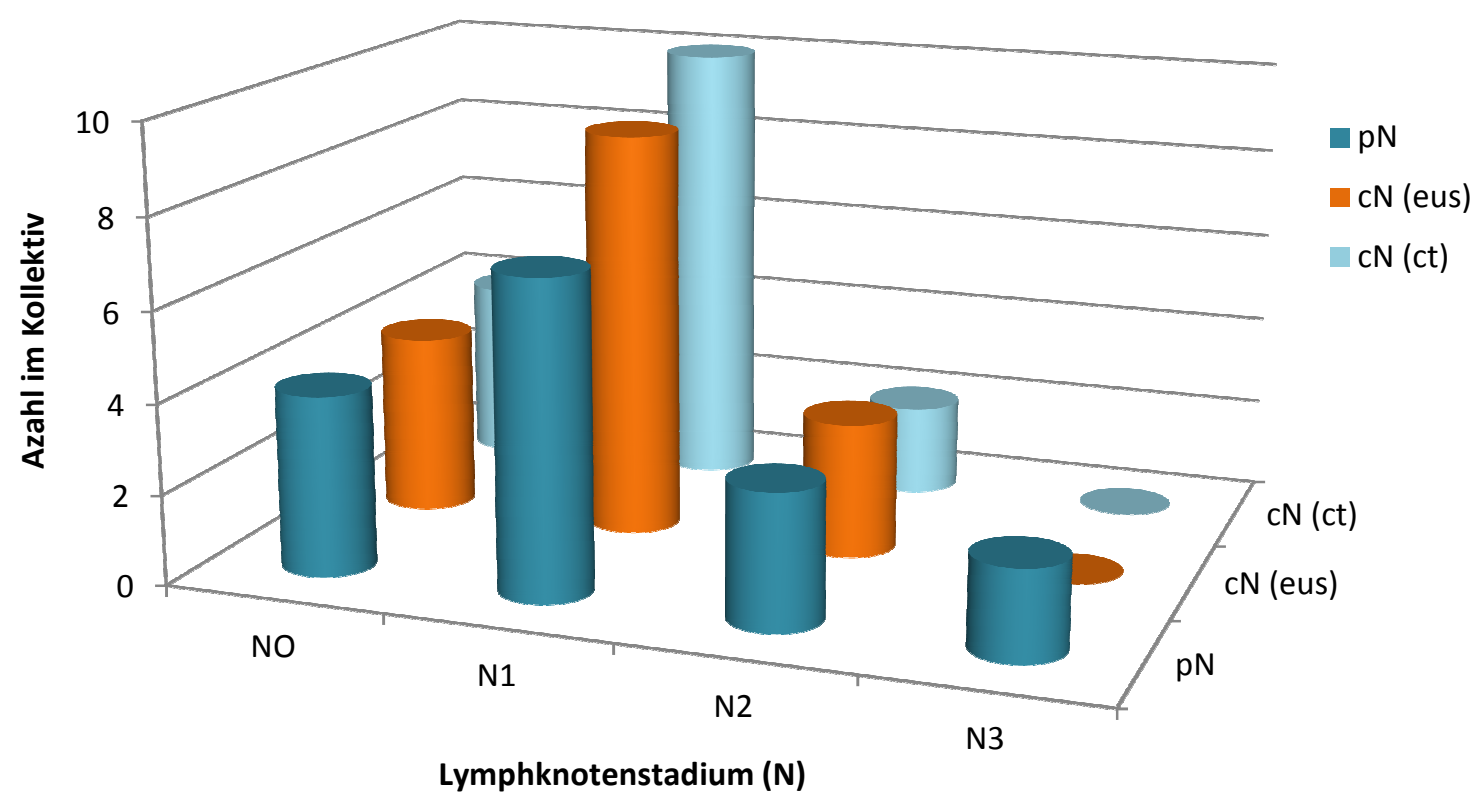

Abb. 11: Anzahl der diagnostizierten N-Stadien in endosonographischer und computertomographischer Untersuchung sowie im Operationresektat entsprechend UICC-Richtlinien (2002)

Oben genannte Daten belegen ausschließlich die Häufigkeit der N-Stadien entsprechend unterschiedlicher Untersuchungsmethoden. Einen Überblick über die Richtigkeit der Ultraschall- und CT-Untersuchung bezüglich des UICC-Stadiums des Lymphknotenbefalls geben Abbildung 12 sowie Tabellen 9 und 10. 


\section{Übereinstimmung im N-Stadium}

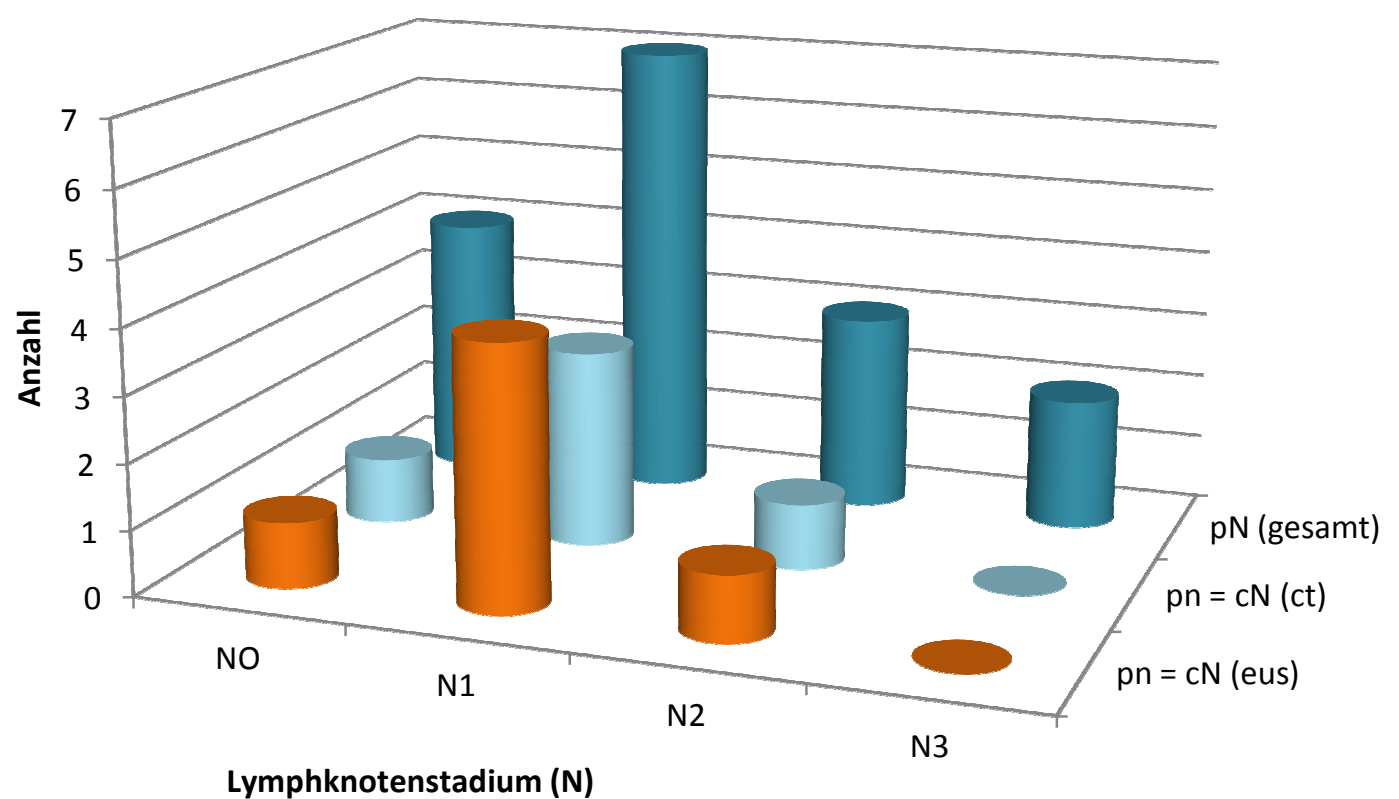

Abb.12: Richtigkeit der Lymphknotenstadien entsprechend EUS- und CT-Diagnostik im Vergleich mit der histopathologischen Diagnostik am Operationsresektat. $\mathrm{pN}=\mathrm{cN}$ zeigt Übereinstimmung von cN (eus) bzw cN (ct) mit pN für das angegebene $\mathrm{N}$-Stadium.

$\begin{array}{llllll}\text { cN0 (eus) } & \mathbf{1} & 1 & 1 & 1 & 4 \\ \text { cN1 (eus) } & 3 & 4 & 1 & 1 & 9 \\ \text { cN2 (eus) } & 0 & 2 & 1 & 0 & 3 \\ \text { cN3 (eus) } & 0 & 0 & 0 & 0 & 0\end{array}$

\begin{tabular}{llllll} 
Gesamtzahl & 4 & 7 & 3 & 2 & 16 \\
\hline Korrekt & $25 \%$ & $57,1 \%$ & $33,3 \%$ & $0 \%$ & $\mathbf{3 7 , 5 \%}$ \\
\hline
\end{tabular}

Tab. 9: Übereinstimmung der Lymphknotendiagnostik in der präoperativen endoskopischen Ultraschalluntersuchung $\mathrm{cN}$ (eus) und der pathohistologischen Lymphknotendiagnostik pN 


\begin{tabular}{llcccc}
\hline & $\mathrm{pNO}$ & $\mathrm{pN1}$ & $\mathrm{pN2}$ & $\mathrm{pN3}$ & gesamt \\
\hline $\mathrm{cN0}(\mathrm{ct})$ & $\mathbf{1}$ & 3 & 0 & 0 & 4 \\
$\mathrm{cN1}(\mathrm{ct})$ & 3 & $\mathbf{4}$ & 2 & 1 & 10 \\
$\mathrm{cN2}(\mathrm{ct})$ & 0 & 1 & $\mathbf{1}$ & 0 & 2 \\
$\mathrm{cN3}(\mathrm{ct})$ & 0 & 0 & 0 & 0 & 0 \\
& & & & & \\
Gesamtzahl & 4 & 8 & 3 & 1 & 16 \\
\hline Korrekt & $25 \%$ & $50 \%$ & $33,3 \%$ & $0 \%$ & $\mathbf{3 7 , 5 \%}$ \\
\hline
\end{tabular}

Tab. 10: Übereinstimmung der Lymphknotendiagnostik in der präoperativen computertomographischen Untersuchung $\mathrm{cN}$ (ct) und der pathohistologischen Lymphknotendiagnostik pN

In $37,5 \%$ aller endoskopischen Ultraschalluntersuchungen konnte das N-Stadium in Übereinstimmung zum histopathologischen Staging, also korrekt bestimmt werden.

Die höchste Sensitivität in der Lymphknotendiagnostik kann mit 57,1\% im pN1 Stadium festgestellt werden. Ein ähnliches Resultat lässt sich in der computertomographischen Diagnostik feststellen, wo das pN1-Stadium zu 50\% korrekt diagnostiziert wurde. In Übereinstimmung zur endoskopischen Ultraschalldiagnostik konnte auch hier bei $37,5 \%$ der Patientenfälle eine korrekte präoperative Diagnose des N-Stadiums nach UICC gestellt werden.

\subsection{Operationsdauer}

Zur Bestimmung der Dauer der einzelnen Magenresektionen wurde die auf dem Operationsbericht vermerkte Schnitt-Naht-Zeit zugrundegelegt. Obwohl das Aufbringen eines sterilen Wundverbandes der Definition nach nicht in die Schnitt-NahtZeit fällt, jedoch generell in die Operationsdauer der Berichte eingeschlossen wurde, wurde es bei allen aufgeführten Werten aufgrund unzureichender Reproduzierbarkeit 
in die Dauer des operativen Eingriffes einbezogen. Bei Unvollständigkeit des Operationsberichtes wurde zur Ermittlung der Operationszeit auf das Pflegeprotokoll zurückgegriffen. Die durchschnittliche Dauer aller 16 Operationen betrug 180,75 Minuten $( \pm 33,53)$. Die längste Operation dauerte 240 Minuten (Patient 14), die zweitlängste 220 Minuten (Patient 11), wobei hervorzuheben ist, dass bei Patient 14 zum Zeitpunkt der Operation eine Adipositas permagna aufgrund überhöhter Kalorienzufuhr bestand. Die kürzeste Operation nahm 114 Minuten in Anspruch (Patient 5). Abbildung 13 gibt einen Überblick über die zeitlichen Umfänge der einzelnen Operationen.

\section{Operationsdauer}

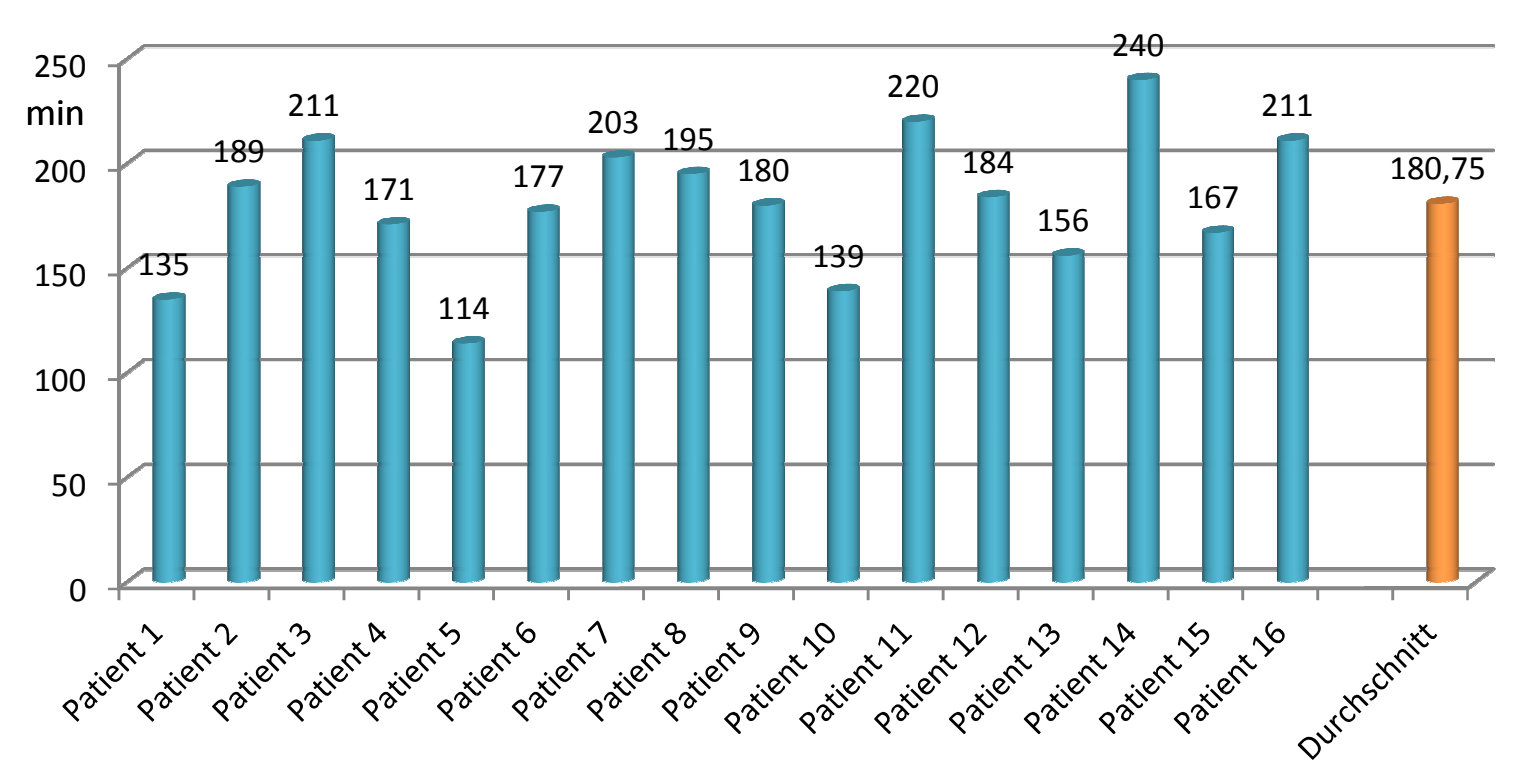

Abb.13: Dauer des operativen Eingriffes für alle Patienten und im Durchschnitt

Abbildung 14 zeigt die Operationsdauer im Verhältnis zum Ausmaß der Lymphadenektomie innerhalb des Patientenkollektivs. Patienten, bei denen eine D2Resektion erfolgte (14 Fälle), wurden durchschnittlich 186,21 Minuten (Minimum 113 Minuten, Maximum 240 Minuten, $\pm 30,25$ ) operiert. In jeweils einem Fall erfolgte eine D2- bzw. D3-Lymphadenektomie. Die Dauer der D1-Reseketion (Patient 5) betrug dabei mit 114 Minuten 64,8\% der durchschnittlichen Operationszeit aller D2Lymphadenektomien innerhalb des Patientenkollektivs. Die Operationsdauer für die 
D3-Resektion (Patient 4) beträgt mit 171 Minuten rund 97,2\% des Durchschnitts aller D2-Resektionen, entsprechend einer zeitlichen Differenz von 5 Minuten.

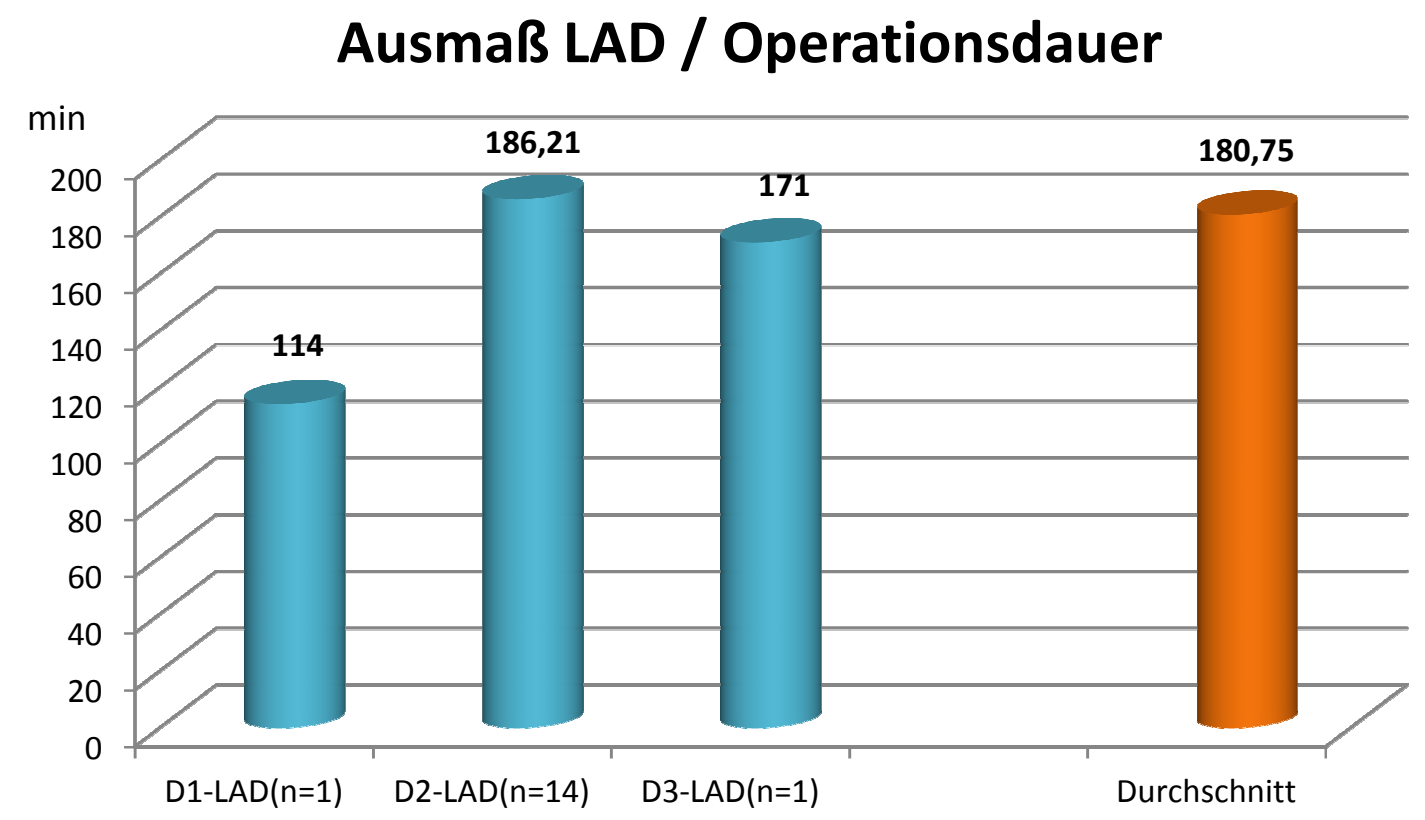

Abb. 14: Verhältnis zwischen Operationszeit und Ausmaß der chirurgischen Radikalität

\subsection{Patientenüberleben}

Wie Tabelle 11 zeigt, trat bei 10 Patienten innerhalb des Patientenkollektivs mit totaler oder transhiatal erweiterter Gastrektomie (62,5\%) im Zeitraum zwischen dem letzten postoperativen Zyklus der perioperativen Chemotherapie und dem Zeitpunkt der Auswertung ein Rezidiv auf. Bei 7 Patienten (43,75\%) mit postoperativem Rezidiv wurde der Tumor dem pathologischen Befund zufolge in sano reseziert, bei den anderen drei Patienten (18,75\%) konnte operativ keine Tumorfreiheit erreicht werden.

Von 16 Patienten nach Gastrektomie sind bis zum Zeitpunkt der Analyse bei einer durchschnittlichen Nachbeobachtungszeit von 722,2 Tagen zehn Patienten (62,5\%) verstorben. Eine Übersicht über das Patientenüberleben in den ersten 18 Monaten 
nach Gastrektomie gibt Abbildung 15. Der darin eingeschlossene Beobachtungszeitraum ergibt sich aus der Zeitspanne zwischen der Operation des zuletzt operierten und noch lebenden Patienten und dem Zeitpunkt der Analyse. Nach 18 postoperativen Monaten waren 7 Patienten verstorben, was einem Anteil von 43,75\% des Kollektivanteils mit Gastrektomie $(n=16)$ entspricht. Aus Tabelle 11 kann entnommen werden, dass eine Beziehung zwischen der individuellen „lymph node ratio“ und dem Überlebenszeitraum nach Operation besteht. Innerhalb der Patienten, die in den ersten 18 Monaten nach Gastrektomie verstorben sind (7 Fälle) kann eine durchschnittliche „Iymph node ratio“ von 33\% festgestellt werden. Patienten des Kollektivs, die länger als 18 Monate postoperativ überlebten oder zum Zeitpunkt der Analyse leben (9 Fälle), zeigen eine durchschnittliche „lymph node ratio“ von 5,8\%. Entsprechend 8.5 .2 beträgt die durchschnittliche Relation von entfernten zu befallenen Lymphknoten im Gesamtkollektiv 17,7\%.

In Abbildung 17 lässt sich ein Zusammenhang zwischen einer niedrigen „lymph node ratio" und einem längeren Überleben nach Operation feststellen. Einbezogen sind alle zum Zeitpunkt der Auswertung verstorbenen Patienten des Kollektivs.

\section{Patientenüberleben nach Gastrektomie}

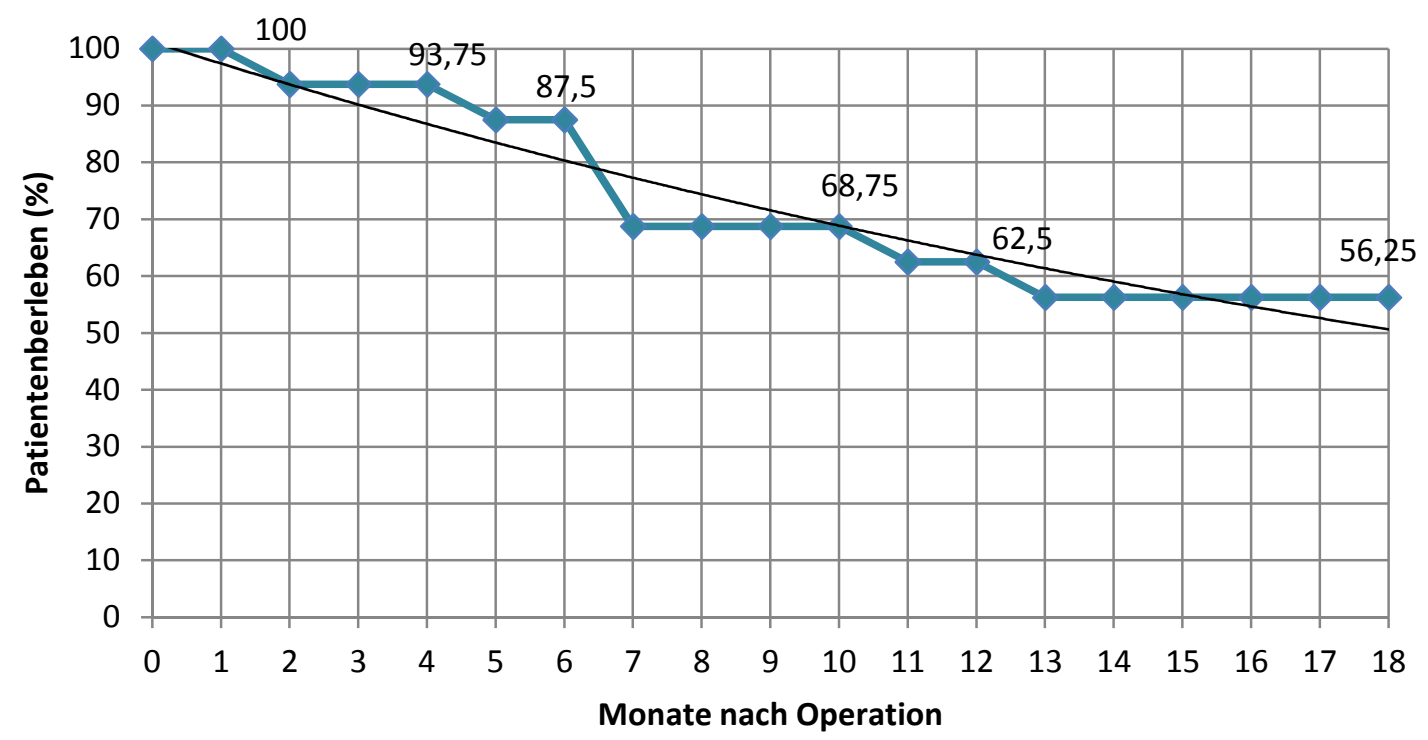

Abb. 15: Patientenüberleben in den ersten 18 postoperativen Monaten. 


\section{Monats-Überleben MAGIC-Studie / Göttinger Kollektiv}

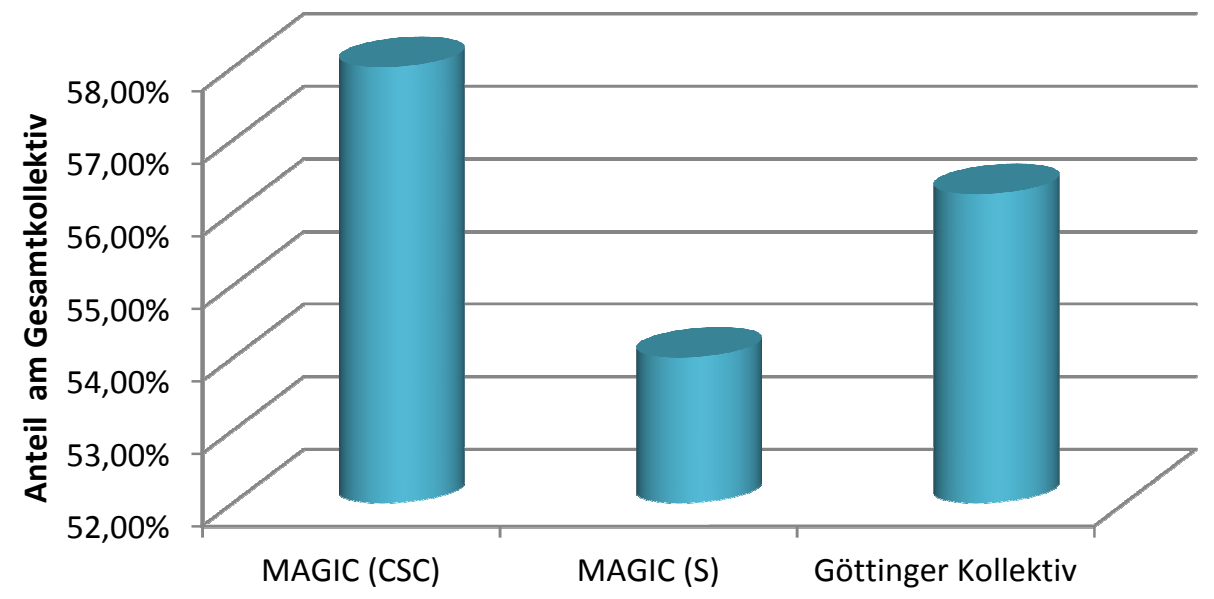

Abb. 16: Anteil der Patienten der MAGIC-Studie und des Göttinger Kollektivs mit einem postoperativem Überleben von 18 Monaten oder mehr / MAGIC (CSC) = chirurgisch und perioperativ chemotherapierte Patientengruppe/ MAGIC $(S)$ = rein chirurgisch behandelte Patientengruppe

\section{Postoperatives Überleben / lymph node ratio}

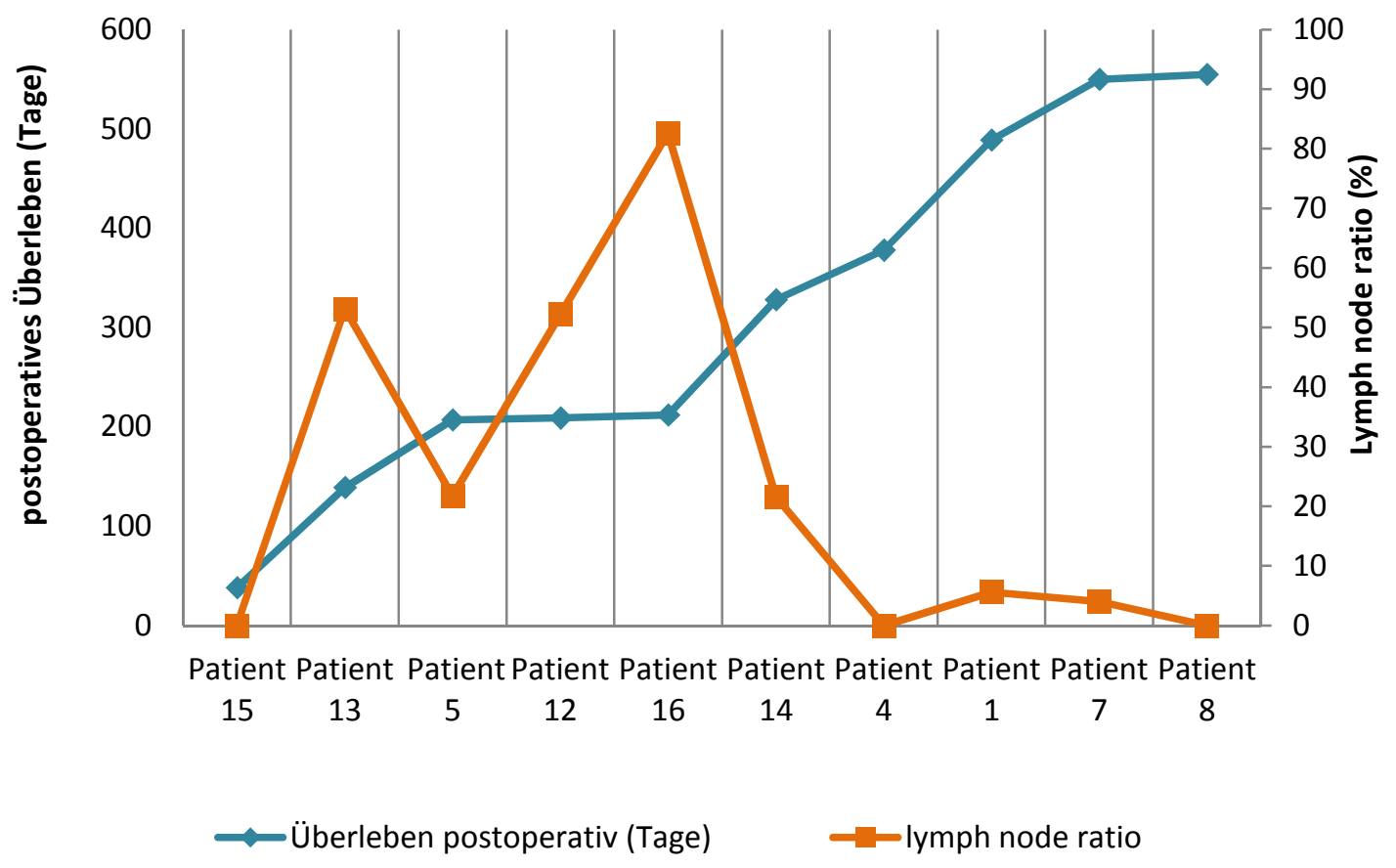

Abb. 17: Überlebenszeitraum postoperativ (in Tagen) relativ zur „Iymph node ratio“ der verstorbenen Patienten des Kollektivs 


\begin{tabular}{|c|c|c|c|c|c|c|c|}
\hline Patient & $\begin{array}{c}\text { UICC- } \\
\text { Klassifikation } \\
\text { nach präop. } \\
\text { Chemotherapie } \\
\text { (pTNM) }\end{array}$ & $\begin{array}{c}\text { LAD- } \\
\text { Ausmaß }\end{array}$ & $\begin{array}{c}\text { entfernte/ } \\
\text { befallene } \\
\text { Lymphknoten } \\
\text { (lymph node } \\
\text { ratio) } \\
\end{array}$ & Resektion & $\begin{array}{c}\text { OP- } \\
\text { Dauer } \\
\text { (Minuten) }\end{array}$ & $\begin{array}{l}\text { Lokal- } \\
\text { Rezidiv }\end{array}$ & $\begin{array}{c}\text { lebend/ } \\
\text { verstorben } \\
\text { (Tage } \\
\text { postop) }\end{array}$ \\
\hline 1 & pT2b,pN1,pMx & D 2 & $\begin{array}{l}18 / 1 \\
(5,6 \%)\end{array}$ & R 0 & 135 & + & $\begin{array}{l}\text { verstorben } \\
(489 \mathrm{~d})\end{array}$ \\
\hline 2 & pT2b,pN1,pM0 & D 2 & $\begin{array}{c}29 / 3 \\
(10,3 \%)\end{array}$ & R 0 & 189 & - & $\begin{array}{l}\text { Lebend } \\
\text { (526 d fu) }\end{array}$ \\
\hline 3 & pT3,pN1,pM1 & D 2 & $\begin{array}{l}15 / 3 \\
(20 \%)\end{array}$ & R 0 & 211 & + & $\begin{array}{l}\text { Lebend } \\
\text { (537 d fu) }\end{array}$ \\
\hline 4 & pT1, pN0,pMx & D 3 & $\begin{array}{c}27 / 0 \\
(0 \%)\end{array}$ & R 0 & 171 & + & $\begin{array}{l}\text { verstorben } \\
(378 d)\end{array}$ \\
\hline 5 & pT3, pN1, pMx & D 1 & $\begin{array}{c}23 / 5 \\
(21,7 \%)\end{array}$ & R 1 & 114 & + & $\begin{array}{c}\text { verstorben } \\
\text { (207 d) }\end{array}$ \\
\hline 6 & $\mathrm{pT} 4, \mathrm{pN} 1, \mathrm{pM} 1$ & D 2 & $\begin{array}{l}29 / 1 \\
(3,4 \%)\end{array}$ & R 1 & 177 & + & $\begin{array}{l}\text { Lebend } \\
\text { (658 d fu) }\end{array}$ \\
\hline 7 & pT2b,pN1,pMx & D 2 & $\begin{array}{l}25 / 1 \\
(4 \%)\end{array}$ & R 0 & 203 & + & $\begin{array}{l}\text { verstorben } \\
(550 \mathrm{~d})\end{array}$ \\
\hline 8 & pT2a,pN0,pMx & D 2 & $\begin{array}{c}30 / 0 \\
(0 \%)\end{array}$ & R 0 & 195 & - & $\begin{array}{c}\text { verstorben } \\
\text { (555 d) }\end{array}$ \\
\hline 9 & pT1,pN0,pMx & D 2 & $\begin{array}{c}43 / 0 \\
(0 \%)\end{array}$ & R 0 & 180 & - & $\begin{array}{l}\text { Lebend } \\
\text { (699 d fu) }\end{array}$ \\
\hline 10 & pT2b,pN1,pMx & D 2 & $\begin{array}{l}35 / 3 \\
(8,6 \%)\end{array}$ & R 0 & 139 & - & $\begin{array}{l}\text { Lebend } \\
\text { (906 d fu) }\end{array}$ \\
\hline 11 & pT2a,pN0,pMx & D 2 & $\begin{array}{c}56 / 0 \\
(0 \%)\end{array}$ & R 0 & 220 & - & $\begin{array}{l}\text { Lebend } \\
\text { (358 d fu) }\end{array}$ \\
\hline 12 & pT2b,pN2,pMx & D 2 & $\begin{array}{l}23 / 12 \\
(52,2 \%)\end{array}$ & R 0 & 184 & + & $\begin{array}{c}\text { verstorben } \\
(209 \mathrm{~d})\end{array}$ \\
\hline 13 & pT3, pN3,pM1 & D 2 & $\begin{array}{l}32 / 17 \\
(53,1 \%)\end{array}$ & R 0 & 156 & + & $\begin{array}{l}\text { verstorben } \\
\text { (139 d) }\end{array}$ \\
\hline 14 & pT2b,pN2,pMx & D 2 & $\begin{array}{c}37 / 8 \\
(21,6 \%)\end{array}$ & R 0 & 240 & + & $\begin{array}{l}\text { verstorben } \\
\text { (328 d) }\end{array}$ \\
\hline 15 & pT2a,pN0,pM0 & D 2 & $\begin{array}{c}21 / 0 \\
(0 \%)\end{array}$ & R 0 & 167 & - & $\begin{array}{l}\text { verstorben } \\
(38 \mathrm{~d})\end{array}$ \\
\hline 16 & pT3, pN3,pMx & D 2 & $\begin{array}{l}23 / 19 \\
(82,6 \%)\end{array}$ & R 1 & 211 & + & $\begin{array}{l}\text { verstorben } \\
(212 \mathrm{~d})\end{array}$ \\
\hline
\end{tabular}

Tab. 11: Lebensstatus und Rezidivrate des Kollektivs/ $d=$ Tage / fu= follow-up 


\section{Rezidivrate nach Gastrektomie}

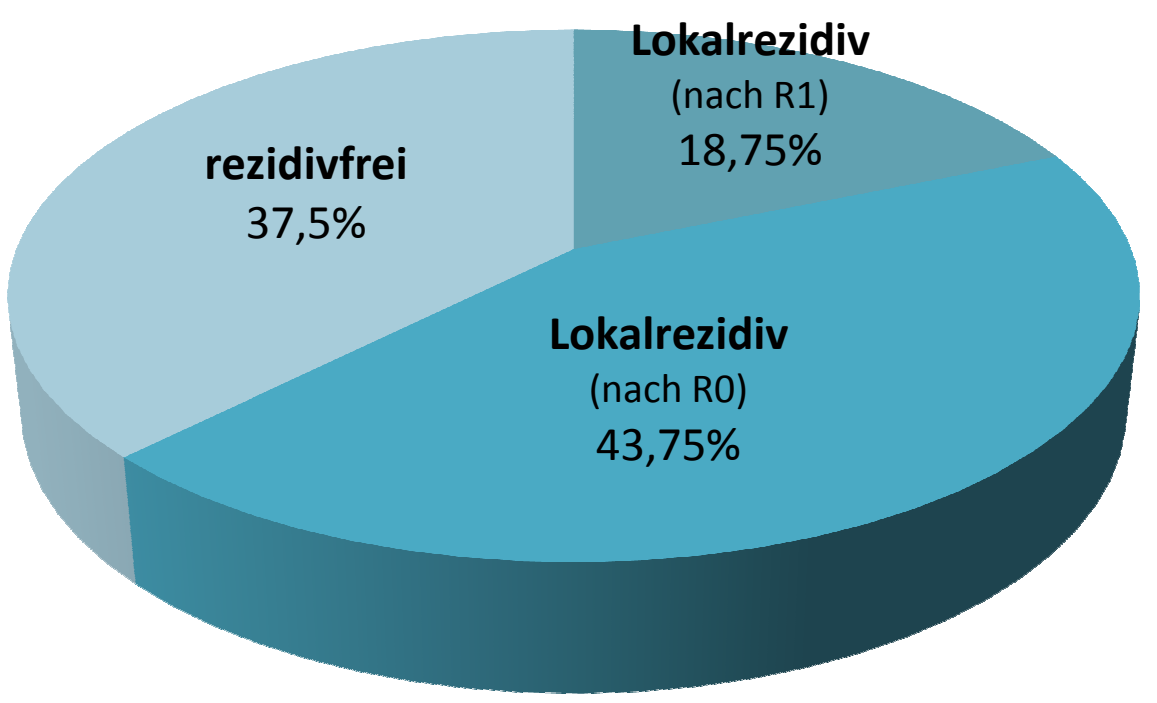

Abb. 18: prozentuales Verhältnis von Rezidivfreiheit zu Lokalrezidiven

Abbildung 18 lässt den Anteil an Patienten mit diagnostiziertem Lokalrezidiv zum Zeitpunkt der Auswertung überblicken. Der Lebensstatus der Patienten ist dabei unberücksichtigt. 62,5\% (10 Fälle) weisen einen postoperativen Rezidivbefund auf, davon lag bei 3 Patienten (18,75\% des Kollektivs) postoperativ eine R1-Situation vor.

9. Diskussion

\subsection{Perioperative Chemotherapie}

In der von Cunningham et al. 2006 publizierten Auswertung der MAGIC-Studie konnte ein hoch signifikanter 5-Jahres-Überlebensvorteil für Patienten mit perioperativ verabreichter Chemotherapie relativ zu Patienten mit alleiniger Operation $(36 \%$ vs. $23 \%$ ) aufgezeigt werden. Dadurch konnte diese groß angelegte randomisierte Phase- 
III-Studie den prognostischen Vorteil eines neoadjvanten Therapiekonzeptes andeuten (Cunningham et al. 2006).

In Anlehnung an das vielversprechende perioperative Therapieschema der MAGICStudie wurden zwischen November 2007 und Juli 2010 im Zentrum für Innere Medizin des Universitätsklinikums der Georg-August-Universität Göttingen 17 Patienten mit lokal fortgeschrittenem Magenkarzinom nach neoadjuvanter Konzeption behandelt. Abweichungen zwischen dem Therapiekonzept von Cunningham et al. (2006) und dem Göttinger Therapiekonzept bestehen in den verwendeten Chemotherapieschemata, der Anzahl der prä- und postoperativen Zyklen, den Zeitabständen zwischen den Therapieabschnitten sowie dem Ausmaß der chirurgischen Tumorresektion.

\subsubsection{Verwendete Chemotherapieschemata}

Die Arbeitsgruppe um Cunningham setzt in der MAGIC-Studie eine Chemotherapeutikakombination aus Epirubicin (50 mg/m² Körperoberfläche (KO)), Cisplatin (60 mg/m² KO) und Fluorouracil (200 mg/m² KO) ein. Dabei wurden Epirubicin und Cisplatin am ersten Tag jedes dreiwöchigen Zyklus als intravenöse Kurzinfusion und Fluorouracil kontinuierlich über 21 Tage mittels portabler Infusionspumpe und dauerhaftem zentralvenösem Kathetersystem verabreicht. Das bevorzugte Göttinger Schema setzte sich aus Epirubicin (50 mg/m² KO), Oxaliplatin (130 mg/m² KO) und Xeloda® $\left(625 \mathrm{mg} / \mathrm{m}^{2} \mathrm{KO}\right.$, Wirkstoff Capecitabine) (= EOXSchema), beziehungsweise aus Epirubicin, Oxaliplatin und Fluorouracil $\left(200 \mathrm{mg} / \mathrm{m}^{2}\right.$ KO) (= EOF-Schema) zusammen. Auf Fluorouracil und damit verbundener 24Stunden-Dauerinfusion und fest implantiertem Katheter wurde bei intakter Darmpassage zugunsten von Xeloda® in Tablettenform verzichtet, was als begünstigender Faktor für die Komplettierungsrate des Chemotherapieplans angesehen werden kann. Von 17 Patienten des Kollektivs konnten 15 Patienten die perioperative Chemotherapie nach festgelegter Planung empfangen, wobei ein Patient aufgrund eines stark reduzierten Allgemeinzustandes auf die postoperative Chemotherapie verzichten musste und ein Patient 38 Tage postoperativ. Die Tatsache, dass in der CSC-Gruppe der Studie von Cunningham et al. 8 von 250 Patienten (3.2\%) die prä- oder postoperative Chemotherapie aufgrund von Problemen 
mit dem verwendeten Hickman-Katheteter zur 24-stündigen Dauerinfusion abbrechen mussten, zeigt, dass Xeloda® (Wirksoff Capecitabine) als Prodrug von Fluorouracil durch seine unkomplizierte Verabreichung mittels Kurzinfusion bei vergleichbarer Wirkung wie Fluorouracil (Möhler und Galle 2007) innerhalb unseres Patientenkollektivs mit Berechtigung bevorzugt verabreicht wurde. Durch den Verzicht auf eine Langzeitinfusion mit implantiertem Katheter kann nicht nur ein erhöhtes Risiko von Hautinfektionen und Subclaviavenenthrombosen umgangen werden, auch die Lebensqualität der Patienten während der Chemotherapie ist als höher einzustufen.

Der Vorzug des Platin-Derivats Oxaliplatin gegenüber dem verwandten Cisplatin in unserem Therapieschema begründet sich durch positive Erfahrungen innerhalb unseres Zentrums in der Therapie des kolorektalen Karzinoms, bezogen auf die niedrige Rate an Nebenwirkungen und die geringere Toxizität. Zwar ist der Einsatz von Oxaliplatin zur Therapie des Magenkarzinoms bislang wenig untersucht, konnte aber in einer ersten Studie vielversprechende Tumorreduktionsraten zeigen (Louvet et al. 2002). Auch vor dem Hintergrund der hohen Ansprechrate in unserem Kollektiv ist das verwendete Chemotherapieschema zu favorisieren. Bei 15 Patienten $(88,2 \%)$ unseres Kollektivs konnte nach den präoperativen Therapiezyklen entsprechend ÖGD- bzw. EUS-Befund eine zumindest partielle Remission des Primärtumors festgestellt werden, während sich in verschiedenen randomisierten Studien bei Anwendung eines ECF-Schemas lediglich in 49 bis $56 \%$ aller Patientenfälle ein Tumorrückgang zeigte (Ross et al. 2002, Webb et al.1997). Die insgesamt als hoch einzustufende Gefahr therapiebegleitender Nebenwirkungen von Cisplatin (Hamers et al.1999) deutet sich auch in der MAGIC-Studie an: Von 250 Patienten der CSCGruppe konnten 15 Patienten die prä- oder postoperativen Chemotherapiezyklen aufgrund toxischer Nebenwirkungen nicht nach Plan vollenden (Cunningham et al. 2006).

\subsubsection{Anzahl der Therapiezyklen}

In den meisten Fällen (11 Fälle) erfolgte die perioperative Chemotherapie in Anlehnung an die Behandlungskonzeption der MAGIC-Studie in 3 prä- und 3 postoperativen Zyklen. In individueller Abstimmung mit Patientenkonstitution, Verträglichkeit und Tumoransprechen variierte die Zahl der präoperativen Zyklen 
zwischen 2 und 5, wodurch in 15 von 17 Patientenfällen ein präoperatives DownSizing des Primärtumors erzielt werden konnte.

Postoperativ wurden in der Regel 3 Zyklen Chemotherapie nach EOX- oder EOFSchema angestrebt. In einem Fall konnte die postoperative Chemotherapie aufgrund eines stark reduzierten Allgemeinzustandes nicht durchgeführt werden, im Falle eines anderen Patienten wurde die Anzahl der postoperativen Zyklen bedingt durch den Verdacht auf ein Lokalrezidiv auf 4 erhöht. Der Vollständigkeit halber muss erwähnt werden, dass die postoperative Chemotherapie eines Patienten (Patient 5) bei intraoperativ gesicherter Peritonealkarzinose und limitierter D1-LAD eher unter palliativen als unter adjuvanten Gesichtspunkten betrachtet werden muss, obwohl auch in diesem Fall der körperliche Allgemeinzustand eine systematische postoperative Chemotherapie zuließ.

An dieser Stelle sollte Anmerkung finden, dass, entsprechend der aktuellen TNMKlassifikation maligner Tumoren aus dem Jahr 2010 (Wittekind und Meyer 2010), Kardiakarzinome der Klassen AEG I und II den distalen Ösophaguskarzinomen zuzuordnen sind. Eine präoperative rein chemotherapeutische Behandlung des Patienten könnte in diesen Fällen durch eine kombinierte Radiochemotherapie ersetzt werden.

\subsubsection{Zeitabstände}

Cunningham et al. (2006) nennen für den Abstand zwischen neodadjuvanter Therapie, chirurgischer Tumorresektion und adjuvanter Therapie einen Zeitraum von 3 bis 6 Wochen präoperativ (entspricht 21 bis 42 Tagen) bzw. 6 bis 12 Wochen postoperativ (entspricht 42 bis 84 Tagen). Innerhalb unseres Kollektivs vergingen zwischen dem letzten Zyklus der präoperativen Chemotherapie und der Operation im Durchschnitt 30,75 Tage, entsprechend der präoperativen Zeitspanne der MAGIC-Studie. Im Falle eines Patienten unseres Kollektivs betrug der zeitliche Abstand zwischen letztem Chemotherapiezyklus und der Operation 57 Tage, wodurch die Wahrscheinlichkeit einer erneuten Größenzunahme des Primärtumors nach temporärer zytostatikainduzierter Remission und damit ungünstigerer Resektabilität ansteigt. Im Gegensatz dazu steht mit 6 Tagen das kürzeste chemotherapiefreie Zeitintervall vor der Operation. Dabei ist nicht auszuschliessen, dass sich durch eine relativ kurze 
Regenerationsphase der Allgemeinzustand des Patienten für den chirurgischen Eingriff nicht ausreichend stabilisiert hat, das Operationsresultat somit unter dem Behandlungsoptimum liegen kann. Für einen ungünstigen prognostischen Einfluss eines zu kurzen Abstandes zwischen präoperativer Chemotherapie und Operation spricht, dass der Patient mit der zweitkürzesten präoperativen Regenerationsphase (Patient 1, 15 Tage) aufgrund eines stark reduzierten Allgemeinzustandes keine adjuvante Chemotherapie empfangen konnte

Die postoperative Zeitspanne bis zum ersten Zyklus der adjuvanten Chemotherapie zeigt mit Abständen von 24 bis 89 Tagen in Abhängigkeit der individuellen Patientenkonstitution eine große Variation, liegt aber mit durchschnittlich 47,5 Tagen innerhalb der von Cunningham et al. (2006) eingehaltenen Therapieabständen von 6 bis 12 Wochen nach der Operation.

Beim Vergleich zwischen Radikalität der chirurgischen Intervention, bestimmt durch Ausmaß der LAD, Anzahl der entfernten Lymphknoten und Operationsdauer, und der Zeitspanne zwischen Operation und adjuvanter Chemotherapie, lässt sich innerhalb unserer Patientengruppe allerdings keine direkte Beziehung feststellen.

Innerhalb unseres Kollektivs konnten 2 von 17 (11,8\%) operierten und präoperativ chemotherapierten Patienten die postoperative Chemotherapie aufgrund eines reduzierten Allgemeinzustandes ( 1 Fall) sowie postoperativer Letalität nach 38 Tagen (1 Fall) nicht nach Plan empfangen.

Im Vergleich dazu konnten in der MAGIC-Studie 34\% der Patienten der CSC-Gruppe nach präoperativer Chemotherapie und Operation keine postoperative Chemotherapie beginnen, zumeist bedingt durch schnelle Tumorprogression oder operationsabhängige Komplikationen (Cunningham et al. 2006).

\subsubsection{Tumorremission unter neoadjuvanter Chemotherapie}

Bei 15 von 17 Patienten (88,2\%) des Kollektivs konnte unter neoadjuvanter Chemotherapie im Vergleich zwischen endosonographischem, endoskopischem und computertomographischem Initialbefund und einem Restaging nach allen präoperativen Zyklen der Chemotherapie ein Down-Sizing des Primärtumors festgestellt werden. Bei 11 von 17 Patienten $(64,7 \%)$ wurde in der präoperativen CT- 
Diagnostik im Rahmen des Restagings zudem ein rückläufiger Größenbefund der lokoregionären Lymphknoten beschrieben.

Cunningham et al. (2006) nennen für den Anteil der am Operationsresektat pathologisch gesicherten Tumoren der UICC-Kategorien pT1 und pT2 in der CSCGruppe 51,7\%, während sich in unserem Kollektiv bei 68,8\% (11 Fälle) der Patienten mit Gastrektomie $(n=16)$ der resezierte Primärtumor den Kategorien pT1 oder pT2 zuordnen ließ. Davon beschränkte sich bei 5 dieser Patienten (31,3\%) die lokale Tumorausdehnung nach komplettierter präoperativer Chemotherapie auf Mucosa oder Submucosa.

Bei 2 Patienten (11,8\%) konnte durch 3 neoadjuvante Zyklen nach EOF- bzw. EOXSchema entsprechend des endosonographischen Befundes lediglich eine weitere Größenzunahme des Primärtumors verhindert werden.

Bei Gegenüberstellung von Tumorremission (8.4) und postoperativer Rezidivrate (8.9) kann festgestellt werden, dass ein endosonographisch und endoskopisch festgestellter regressiver Größenbefund des Primärtumors der Entstehung eines Lokalrezidivs nicht entgegensteht. Innerhalb der 16 Patienten, bei denen eine Gastrektomie durchgeführt werden konnte $(n=16)$, zeigt sich eine Remissionsrate von 93,75\% (15 von 16 Fällen) gegenüber einer Lokalrezidivrate von 62,5\% (10 von 16 Fällen). Eine Erklärung lässt sich in der Inhomogenität der Tumorzellpopulation suchen: Während eine endoskopisch und endosonographisch festgestellte scheinbar oberflächliche Tumorreduktion durch den Untergang peripherer chemosensiblerer Zellen zu begründen ist, geht eine erneute Zunahme maligner Zellen von therapieresistenteren zentralen Zellgruppen aus. Dadurch stellt sich die Frage, ob drei präoperative Therapiezyklen für eine verlaufsrelevante Beeinflussung der Tumorresektabilität ausreichen oder ob zusätzliche präoperative Zyklen weiter zentral lokalisierte Tumoranteile reduzieren könnten. Des Weiteren wird dadurch die Bedeutung des Abstandes zwischen dem letzten Zyklus der präoperativen Chemotherapie und der Operation deutlich. Mit einer durchschnittlichen Zeitspanne von 30,75 Tagen liegt unser Kollektiv zwar im vorgegebenen Rahmen der MAGIC-Studie (21-42 Tage), eine engere Positionierung der beiden Therapieschritte könnte aber, unter der Voraussetzung eines stabilen Allgemeinzustandes des Patienten, in einer kompletteren Resektionen maligner Zellgruppen mit verringerter Rezidivrate resultieren. 
Zur Beurteilung des Therapieansprechens eines soliden Tumors wurde im Jahr 2000 erstmals RECIST (Response Evaluation Criteria in Solid Tumors) vorgestellt (Therasse et al. 2000). Eine Überarbeitung erfolgte im Januar 2009 (Eisenhauer et al. 2009). Mit Hilfe definierter Richtlinien soll in klinischen Studien ein Tumoransprechen auf angewendete Therapiemaßnahmen objektiv und quantitativ erfasst werden.

Für eine angemessene Beurteilung des Tumoransprechens innerhalb des Patientenkollektivs erweisen sich die allgemein angewendeten RECIST-Richtlinien als ungeeignet. Eine genaue Abgrenzung des zu beurteilenden Tumorareals inklusive möglicher peripherer Entzündung stellt sich bei rein linearer Abmessung des CTmorphologischem Korrelats, wie es für eine Evaluierung des Therapieansprechens nach RECIST-Kriterien nötig ist, als schwierig dar. Für eine präzisere Bewertung der Tumorausdehnung wäre dabei eine funktionelle Bildgebung im Sinne einer ergänzende Positronen-Emissions-Tomographie (PET) nötig (O'Connel 2004). Die Verlaufsbewertung nach RECIST-Richtlinienlässt zudem nur unzureichende Aussagen zu Form, Struktur und Oberflächenbeschaffenheit der Zielläsion zu (Ratain und Eckhardt 2004).

Als weiterer Nachteil der RECIST-Kriterien ist die Beschränkung der messbaren Läsionen auf minimal $10 \mathrm{~mm}$ anzusehen, wobei sich die Frage stellt, ob eine klinische Differenzierung zwischen nicht-messbaren Läsionen und Läsionen, die computertomographisch erfasst werden können eine adäquate Bewertung des Therapieansprechens zulassen. In 4 Fällen unserer Patientengruppe (23,5\%) war nach neoadjuvanter Chemotherapie CT-morphologisch kein Primärtumor mehr nachweisbar, was sich bei der postoperativen pathologischen Beurteilung des Gastrektomiepräparats als nicht-zutreffend erwies.

Bei der Darstellung kleiner, aber messbarer Läsionen kann der Effekt des „volume averaging" eine präzise Beurteilung der Läsion unmöglich machen. Auf Grund einer Maximalauflösung von CT-Darstellungen bilden rechteckige Voxel (als dreidimensionales Äquivalent eines Pixels) dabei in ihrer Farbwiedergabestufe einen Durchschnitt aller empfangenen Bildinformationen des aufgenommenen Gewebebereichs. Grenzen kleinerer Zielläsionen werden dadurch unscharf und können nur unzureichend zur Bestimmung der Tumorausdehnung verwendet werden (Jaffe CC 2006). 
Eine relativ grobe Beurteilung der Wachstumsaktivität von soliden Tumoren durch Zuordnung zu ruhender Läsion (SD, Stable Disease) oder fortschreitender Läsion (PD, Progressive Disease) erweist sich für die Einordnung in die klinische Routine als vorteilhaft, sollte für eine präzise Beurteilung des Ansprechens auf Chemotherapeutika allerdings eine feinere Abstufung zeigen (Jaffe CC 2006).

Eine Alternative zu RECIST kann in einer kombinierten Anwendung aus endoskopischem Ultraschall (EUS) und Ösophagogastroduodenoskopie (ÖGD) gesehen werden. Verschiedenen Studienergebnissen ist zu entnehmen, dass EUS konstant hohe Sensitivitätsraten in der Beurteilung der Primärläsion zeigt und zudem ein wichtiges Hilfsmittel bei der onkochirurgischen Planung darstellt, vor allem um zwischen operablen und nicht operablen Karzinomen zu unterscheiden (Mortensen et al. 2007, Chen et al. 2002). Eine aktuelle südkoreanische Studie zeigt zudem eine Überlegenheit von EUS gegenüber CT in der Beurteilung lokoregionärer Lymphknoten (Hwang et al. 2010). Im Rahmen der Ausbreitungsdiagnostik innerhalb unseres Kollektivs zeigte sich EUS gegenüber CT in diesem Punkt zumindest nicht unterlegen (8.7.3).

Eine zusätzliche Ösophagogastroduodenoskopie (ÖGD) kann als hilfreich zur Beurteilung von Oberfläche und Peripherie des Tumorareals betrachtet werden.

\subsubsection{Postoperative Letalität}

Für die CSC-Patientengruppe der MAGIC-Studie wurde eine postoperative Letalität (definiert als Anzahl der Todesfälle innerhalb von 30 Tagen nach Operation) von 5,6\% (14 Fälle, n=250) angegeben. In unserem Kollektiv ist kein Todesfall festzustellen, der als direkte Folge der Gastrektomie zu deuten wäre. Dabei ist zu bemerken, dass die Resektion in der perioperativ behandelten Gruppe der MAGIC-Studie in nur 42,5\% im Sinne einer radikalen D2-Lymphadenektomie erfolgte, während 93,8\% (15 Fälle, $\mathrm{n}=250$ ) der Tumorresektionen innerhalb unseres Kollektivs nach den Vorgaben einer D2- oder D3-LAD vollzogen wurden. Des Weiteren lässt sich ein Zusammenhang zwischen einer günstigen postoperativen Letalitätsrate und der Tatsache, dass innerhalb unseres Patientenkollektivs nur in einem Fall eine Splenektomie und Pankreaslinksresektion durchgeführt wurde, vermuten. Ein negativer Einfluss von Splenektomie und Pankreaslinksresektion auf den postoperativen Allgemeinzustand 
von Patienten nach Gastrektomie wird von verschiedenen Autoren genannt und sollte in jedem Fall vermieden werden, sofern der Patient nicht auf eine multiviszerale Resektion besteht oder der Tumor genannte Strukturen direkt infiltriert (Wu et al. 2006, Sasako 1997). Nach Okuno at al. (1999) kann eine signifikant verminderte Funktion der T-Lymphozyten nach Entfernung der Milz neben einer erhöhten chirurgischen Belastung des Patienten als ursächlich für eine erhöhte postoperative Morbidität und Mortalität betrachtet werden.

\subsection{Ausmaß der Lymphadenektomie}

Für das optimale Ausmaß der Lymphadenektomie beim fortgeschrittenen Magenkarzinom konnte bisher kein Standard festgelegt werden, der allgemeine Akzeptanz findet. Angetrieben durch die gegensätzlichen Ergebnisse von zwei europäischen Studien aus den Niederlanden (Hartgrink et al. 2004) und Großbritannien (Cuscheri et al. 1999) auf der einen Seite und Studien aus japanischen Zentren (Fujii et al. 2010, Sano et al. 2004, Sano et al. 2002) auf der anderen Seite, erfährt die Diskussion über D1- und D2-Lymphadenektomie weitere Brisanz.

Die 11-Jahres-Ergebnisse der randomisierten niederländischen Studie von Hartgrink et al. (2004) konnten keinen signifikanten Gesamtüberlebensvorteil für Patienten feststellen, die einer D2-Resektion unterzogen wurden (30\% D1 vs. $35 \%$ D2; $p=0,53$ ). Bei Rezidivraten, die sich statistisch ebenfalls nicht voneinander unterscheiden $(70 \%$ D1 vs. $65 \%$ D2; $p=0,43)$, war in der Patientengruppe nach erweiterter Lymphadenektomie eine höhere postoperative Mortalität (4\% D1 vs. 10\% D2; $\mathrm{p}=0,004)$ und Morbidität (25\% D1 vs. 43\% D2; $\mathrm{p}<0,001)$ festzustellen, was auf die hohe Anzahl an Splenektomien und Pankreaslinksresektionen innerhalb der Patientengruppe mit D2-LAD zurückzuführen sein kann. Lediglich eine Subgruppe der Patienten, entsprechend $12 \%$ des Gesamtkollektivs, konnte nach D2-Dissektion einen Prognosegewinn feststellen lassen. Dabei handelte es sich um Patienten, deren Lymphknotenmetastasierung der Kategorie N2 (nach UICC, 2002) zugeordnet werden konnte. Ein Prognosegewinn für Patientensubgruppen findet sich auch in den Ergebnissen der deutschen Magenkarzinomstudie, in der Patienten des 
Tumorstadiums II (nach UICC, 2002) von einer erweiterten Lymphadenektomie profitieren konnten.

In Übereinstimmung mit den Ergebnissen von Hartgrink et al. konnten auch in der randomisierten englischen Studie von Cuscheri et al. (1999) kein Überlebensvorteil für D2-resezierte Patienten aufgezeigt werden, wobei die postoperative Letalität nach D2Resektion mit $13 \%$ doppelt so hoch war wie nach D1-Resektion (6,5\%). Auch die postoperative Morbiditätsrate war mit 46\% nach erweiterter Lymphadenektomie deutlich höher (28\% D1 vs. $46 \%$ D2).

Gegensätzlich zu den beiden großen europäischen Studien zeigen sich Ergebnisse einer randomisierten japanischen Studie zum Ausmaß der Lymphknotendissektion von Sano et al., denen zufolge eine D2-Lymphadenektomie in Einrichtungen mit erfahrenen Operateuren auch ohne Erhöhung der postoperativen Morbidität und Mortalität durchzuführen ist (Sano et al. 2004). Dabei verglich die Arbeitsgruppe um Sano die D2-LAD, wie sie in Japan chirurgischer Standard ist, mit einer erweiterten D2-LAD unter Einbeziehung der para-aortalen Lymphknoten. Die Resultate überzeugen durch eine ausgesprochen niedrige Rate an postoperativer Morbidität und Mortalität. Für das gesamte Patientenkollektiv, also sowohl für Patienten nach D2-, als auch für Patienten nach D2-LAD plus para-aortaler Lymphknotendissektion, konnte eine postoperative Morbidität von $24,5 \%(p=0,67)$ und eine postoperative Letalität von $0,8 \%(p=0,99)$ berichtet werden. Damit liegen die postoperativen Komplikations- und Letalitätsraten sogar unter den angegebenen Werten für Patienten nach D1-LAD in den beiden europäischen Studien (Hartgrink et al. 2004, Cuscheri et al. 1999). Durch die Tatsache, dass es sich bei der Studie von Sano et al. (2004) um die erste japanische Studie handelt, deren Durchführung prospektiv-randomisiert und unter definiertem Datenmanagement und kontinuierlicher Qualitätskontrolle vollzogen wurde, kann den Ergebnissen eine hohe Aussagekraft zugemessen werden. Frühere japanische Studien können die positiven Resultate von Sano et al. mit Letalitätsraten bei D2-LAD unter 2\% untermauern (Sano et al. 2002, Fuji et al. 1999).

Neben den positiven japanischen Werten für operationsabhängige Morbiditäts- und Mortalitätsraten bei D2-LAD spricht auch eine Metaanalyse von Maruyama et al. (1989) für eine erweiterte Lymphknotendissektion. Dabei konnte festgestellt werden, dass 4 bis $23 \%$ aller Patienten mit fortgeschrittenem Magenkarzinom Lymphknotenbefall im 2. Kompartiment aufwiesen. Bei Patienten mit Tumoren der 
Kategorie T3 war in 40\% der Fälle metastatischer Befall der Lymphknoten im 2. Kompartiment festzustellen. Eine Chance auf Tumorfreiheit nach chirurgischer Intervention hätte sich in diesen Fällen nur nach D2-LAD ergeben.

Innerhalb der Patienten unseres Kollektivs, die einer totalen oder transhiatal erweiterten Gastrektomie unterzogen wurden (16 Fälle), konnte im Falle eines Patienten $(6,25 \%)$ mit einem T3-Tumor ein Lymphknoten mit metastatischer Tumorabsiedlung im 2. Kompartiment (Regio Milzhilus) ausgemacht werden. Bei den restlichen Patienten (93,75\%, 15 Fälle) wurden bei der pathohistologischen Aufbereitung des Gastrektomiepräparats ausschließlich perigastrische Lymphknotenmetastasen gefunden. In welchem Ausmaß dabei okkulte Lymphknotenmetastasen in Form isolierter Tumorzellen der histologischen Befundung entgangen sind, bleibt unklar. Durch Ergebnisse einer älteren Studie von Siewert et al (1996) wird allerdings deutlich, dass auch Patienten mit scheinbar tumorfreien Lymphknoten ( $\mathrm{pN} 0$ ) von einer radikalen D2-LAD profitieren, was für eine große prognostische Relevanz eventuell vorhandener Mikrometastasen spricht.

Durch die von Maruyama et al. (1989) angeführten Werte für Lymphknotenbefall im ersten und zweiten Kompartiment wird auch der Einfluss des LAD-Ausmaßes auf das Auftreten von Lokalrezidiven deutlich. In einer Studie von Shchepotin et al. (1995) wurden bei eingeschränkter LAD lokoregionäre Rezidivraten um 40\% angegeben, während eine japanische Arbeitsgruppe nach radikalerer D2-LAD bei nur 22\% der Patienten ein Lokalrezidiv feststellen konnte (Maehara et al. 2000). Auch die Ergebnisse der holländischen Arbeitsgruppe um Hartgrink (2004) zeigen eine nichtsignifikant geringere lokoregionäre Rezidivraten für Patienten nach D2-LAD.

In unserem Kollektiv wurde in 87,5\% (14 Fälle) aller Gastrektomien ( $n=16)$, also der deutlichen Mehrzahl der Patienten, eine D2-Lymphknotendissektion durchgeführt. Lediglich in einem Fall (6,25\%) beschränkte sich die LAD auf perigastrale Lymphknotenstationen, da eine Ausdehnung der Resektion intraoperativ unter Anbetracht einer ausgeprägten Peritonealkarzinose als nicht sinnvoll erachtet und die Operation somit in palliativer Intention fortgesetzt wurde. In einem weiteren Fall $(6,25 \%)$ wurden die Lymphknoten des 3.Kompartiments in das Resektat einbezogen. Wie Tabelle 6 zeigt, wurde bei der Wahl des Resektionsausmaßes das UICCTumorstadium, und damit auch die N-Kategorie, nicht als entscheidender Faktor gewertet. Dies ist nicht nur angesichts der positiven Ergebnisse der aufgeführten 
japanischen Studien (Sano et al. 2002 , Sano et al. 2004) zu vertreten, sondern scheint vor dem Hintergrund, dass die Subgruppen, die in der niederländischen Studie (N-Kategorie 2) beziehungsweise der deutschen Magenkarzinomstudie von Siewert et al. (1998) (UICC-Stadium II) von einer D2-LAD eindeutig profitieren können, präoperativ nur mit großer Unsicherheit auszumachen sind. Eine höhere Genauigkeit in der Zuordnung des Tumorstadiums wäre an dieser Stelle von großer therapeutischer Relevanz.

Mit dem Prinzip der "targeted surgery“ legten Desai et al. (2004) bereits ein vielversprechendes Konzept zur Präzisierung des Ausbreitungsstagings vor. Dabei wird intraoperativ ein Schnellschnitt der ,junctional nodes“, welche den Lymphknotenstationen 4, 5 und 7 (nach JRSGC, Japanese Research Society for Gastric Cancer) entsprechen. Bei positiv ausfallendem Schnellschnitt wird der Patient einer D2-Resektion zugeführt. Sind die junktionalen Lymphknoten nicht befallen, beschränkt sich die LAD auf das 1.Kompartiment. In der Studie von Desai et al. (2004) wurden 205 Patienten in kurativer Intention operiert, davon 87 mit D1-LAD, 92 mit D2LAD und 26 Patienten nach dem Konzept der „targeted surgery“. Die 5-JahresÜberlebensraten der Patienten nach gezielter LAD überzeugten dabei mit $66 \%$ gegenüber 47\% nach D1-LAD und 42\% nach D2-LAD.

Zusammenfassend lässt sich die D2-Resektion unter Einbeziehung genannter Ergebnisse in der klinischen Routine momentan für alle fortgeschrittenen Magenkarzinome als chirurgische Prozedur der Wahl beurteilen. Wie japanische Studien zeigen, sind dabei die Erfahrung des Behandlers und der chirurgischen Zentren sowie die Vermeidung von Splenektomien und Pankreaslinksresektionen entscheidend für die Prognose der Patienten. Zwar war bei der holländischen Studie von Hartgrink et al. (2004) in Person von Mitsuru Sasako ein höchst erfahrener Chirurg anwesend, in welcher Form und Intensität er in das chirurgische Vorgehen einbezogen wurde, bleibt allerdings ungewiss. 


\subsection{Anzahl der entfernten Lymphknoten}

Das Ausmaß des metastatischen Lymphknotenbefalls gilt nach allgemeiner Ansicht als entscheidender Faktor die Prognose des Magenkarzinoms (Hidaka et al. 2008, Xu et al. 2009, Siewert et al. 1998). Auch nach aktueller Einschätzung der UICC (Union Internationale contre le Cancer, 2002) ist beim fortgeschrittenen Magenkarzinom die Anzahl der befallenen Lymphknoten für den Malignitätsgrad der Erkrankung ausschlaggebend. Das veranlasste sie, ihre bis 1997 gültige Klassifikation, bestimmt durch die Lage der befallenen Lymphknoten, zu revidieren (UICC 2002). Dadurch wird deutlich, dass einer adäquaten Dissektion möglichst vieler potentiell tumorinfiltrierter Lymphknoten eine hohe therapeutische Relevanz zukommt.

In unserem Kollektiv wurden, unabhängig von den einbezogenen Kompartimenten, durchschnittlich 29,13 Lymphknoten im Rahmen der Gastrektomie reseziert. Das Minimum der entfernten Lymphknoten lag bei 15, das Maximum bei 56 (Abbildung 10). Innerhalb der Patientengruppe, deren Operation nach den Vorgaben einer D2-LAD durchgeführt wurde (14 Fälle), wurden im Durchschnitt 30,5 Lymphknoten in das Gastrektomiepräparat einbezogen.

Verglichen mit Studienergebnissen aus Japan, insbesondere von Sano et al. (2004), zeigen unsere Ergebnisse, dass es chirurgisch möglich ist, die Anzahl der entfernten Lymphknoten weiter auszubauen. Sano et al. konnten in ihrer Studie aus 2004 bei 263 Patienten mit D2-LAD im Durchschnitt 54 Lymphknoten (maximal 161, minimal 14) entfernen. Auch in der deutschen Magenkarzinomstudie von Siewert et al. (1998) zeigt sich mit durchschnittlich 45,4 entfernten Lymphknoten bei D2-LAD ein relativ höheres Resektionsausmaß. Allerdings handelt es sich bei den Zahlen der angeführten Studien im internationalen Vergleich um Spitzenwerte. Einer amerikanischen Arbeit von Coburn et al. (2006) zufolge, wurden in 10.800 Fällen von D1- und D2-LAD lediglich bei 29\% der Patienten 15 oder mehr Lymphknoten entfernt. Auch nach Umstellung der UICC-Klassifikation zur Bestimmung der N-Kategorie erhöhte sich nach Coburn et al. (2006) die durschschnittliche Anzahl entfernter Lymphknoten lediglich von 9 auf 10.

Neben der Anzahl an resezierten Lymphknoten wird in verschiedenen Arbeiten auch das Verhältnis von entfernten negativen Lymphknoten zu positiven Lymphknoten 
(,lymph node ratio") als verlaufsrelevanter Faktor genannt (Bilici et al. 2009, Xu et al. 2009, Fukuda et al. 2009, Kattan et al. 2003). Danach ist nicht nur eine große Anzahl metastatisch befallener Lymphknoten für den Patienten mit einer schlechteren Prognose verbunden, sondern auch eine geringere Anzahl pathologisch untersuchter, aber nicht befallener Lymphknoten. Eine Erklärung dafür kann in der größeren Menge an entfernten okkulten Mikrometastasen in Form isolierter Tumorzellen gesehen werden. Diese Lymphknoten werden im histopathologischen Befund des Resektats als gesund festgehalten, hätten aber bei Belassen in situ zu einem Tumorrezidiv geführt. Ein Zusammenhang zwischen einer hohen „lymph node ratio“ und längerem postoperativen Überleben deutet sich auch in unserer Patientengruppe an (Abbildung 17).

\subsection{Präzision in der Beurteilung des N-Stadiums bei endoskopischer und computertomographischer Diagnostik}

Zur Auswertung der präoperativen Staginggenauigkeit mittels EUS und CT erfolgte der Vergleich zwischen präoperativen Stagingbefunden und postoperativer histopathologischer Aufarbeitung des Resektats aller Patienten mit erfolgter Gastrektomie $(n=16)$. Eine Übereinstimmung zwischen endosonographischer Ultraschalluntersuchung und histopathologischem Befund lag in 37,5\% (6 Fälle) der Untersuchungen vor. Eine Übereinstimmung von 37,5\% zeigt sich auch zwischen der präoperativen computertomographischen Untersuchung und dem Operationsresektat. Die höchste Stagingpräzision konnte sowohl im EUS als auch bei computertomographischer Genauigkeit im N1 Stadium gefunden werden: in 57,1\% bzw. $50 \%$ konnte das präoperativ bestimmte $\mathrm{N}$-Stadium postoperativ verifiziert werden. Zugleich stellte Stadium N1 mit einem histopathologisch bestätigtem Anteil von $43,75 \%$ am Gesamtkollektiv das am häufigsten vertretene Lymphknotenstadium dar. In den restlichen N-Stadien nach UICC konnte in der vorliegenden Studie eine diagnostische Übereinstimmung zwischen EUS und CT festgestellt werden. Stadium No wurde in $25 \%$, Stadium N2 in $33 \%$ und Stadium N3 in $0 \%$ der Fälle in Korrespondenz zum pathologischen Befund diagnostiziert. 
Eine Überlegenheit von EUS gegenüber CT zeigt sich in einer aktuellen südkoreanischen Studie (Hwang et al. 2010) mit einer Staginggenauigkeit von 66\% bei EUS gegenüber $62,8 \%$ bei $\mathrm{CT}$.

\subsection{Patientenüberleben}

Zum Zeitpunkt der Auswertung waren 10 von 16 Patienten (62,5\%) des Kollektivs nach Gastrektomie verstorben. Bei 8 der 10 verstorbenen Patienten wurde postoperativ ein Rezidiv festgestellt. 7 Patienten (43,75\%) verstarben innerhalb der ersten 18 Monate postoperativ.

Mit einer 18-Monats-Überlebensrate von $56,25 \%$ ist die Differenz zwischen unserem Kollektiv und der CSC-Gruppe der MAGIC-Studie in der nach diesem Zeitraum noch $58 \%$ der Patienten lebten, in diesem Punkt als gering zu beurteilen. In der Gruppe der ausschließlich operierten Patienten der MAGIC-Studie wurde 18 Monate postoperativ eine Überlebensrate von nur $54 \%$ festgestellt. Dabei ist zu bemerken, dass der perioperative Therapieansatz bei Behandlung des Göttinger Kollektivs in die klinische Routine eingebunden wurde und keine Behandlung unter definierten Studienbedingungen wie in der MAGIC-Studie erfolgte.

Cunningham et al. konnten mit fortschreitender postoperativer Beobachtungsdauer auch eine zunehmende Diskrepanz im Anteil der überlebenden Patienten beider Gruppen feststellen. So beträgt die 5-Jahres-Überlebensrate $36,3 \%$ in der CSCGruppe und 23\% in der S-Gruppe. Dementsprechend lässt sich ein Benefit der perioperativen Chemotherapie erst bei einem längeren Beobachtungszeitraum in seiner vollen Tragweite erfassen (Cunningham et al. 2006).

Vergleicht man innerhalb der Gruppe von verstorbenen Patienten den Operationsparameter der „lymph node ratio“ mit der Dauer des Überlebens nach Gastrektomie, zeigt unser Kollektiv einen Zusammenhang zwischen längerem postoperativen Überleben und einer niedrigen „lymph node ratio“. Als verlaufsrelevanter Operationsparameter wurde das Verhältnis von Gesamtanzahl der resezierten Lymphknoten zur Anzahl der davon befallenen Lymphknoten zuvor von 
verschiedenen Autoren genannt (Bilici et al. 2009, Xu et al. 2009, Fukuda et al. 2009, Siewert 2004, Kattan et al. 2003). Nach Siewert (2004) ist eine Prognose dann als günstig einzuschätzen, wenn der Anteil infiltrierter Lymphknoten nicht mehr als $20 \%$ aller untersuchter Lymphknoten beträgt. Innerhalb unserer Patientengruppe waren in durchschnittlich 17,7\% (maximal 82,6\%, minimal 0\%) der Lymphknoten im Gastrektomiepräparat vitale Tumorzellen nachweisbar.

Der in der Literatur genannte Richtwert einer prognostisch günstigen Lymphknotenrate (Siewert 2004) kann auf das Gesamtkollektiv übertragen werden. Mit einer durchschnittlichen postoperativen Beobachtungszeit der lebenden Patienten von 722,2 Tagen (minimal 467, maximal 1005) zeigt sich in keinem der Fälle eine „lymph node ratio", die diesen Wert übersteigt. Zudem spricht eine durchschnittliche Lymphknotenrate von 33\% der Patienten, die innerhalb von 18 Monaten postoperativ verstorben sind, für eine prognostische Aussagekraft dieses Parameters bezüglich des Gesamtüberlebens.

\subsection{Operationsdauer}

Die zeitlichen Umfänge der Gastrektomien zeigen innerhalb unseres Kollektivs eine breite Variation (Abbildung 13). Zwischen der kürzesten (114 Minuten) und der längsten (240 Minuten) Operation liegt eine Differenz von über zwei Stunden. Die durchschnittliche Operationsdauer betrug 180,75 Minuten für das Gesamtkollektiv und 186,21 Minuten bei D2-LAD. Die einzige D1-LAD im Kollektiv beanspruchte eine Zeit von 114 Minuten, während die D3-LAD in 171 Minuten durchgeführt wurde. Angesichts der geringen Anzahl an D1- und D3-Lymphadenektomien lässt sich bei vorliegenden Ergebnissen nur tendenziell eine Abhängigkeit zwischen Operationsdauer und Anzahl der einbezogenen Kompartimente bei der Lymphadenektomie feststellen. Allerdings kann beim Vergleich zwischen Operationsdauer und Anzahl der entfernten Lymphknoten keine proportionale Beziehung festgestellt werden. Der Patient, dessen Operation mit 220 Minuten die am zweitlängste Zeit beanspruchte, wurde zwar gleichzeitig der radikalsten Lymphknotendissektion (Patient 11, 56 entfernte Lymphknoten) unterzogen, 
gleichzeitig wurde aber der Patient mit der niedrigsten Anzahl entfernter Lymphknoten (15 entfernte Lymphknoten) mit einer Schnitt-Naht-Zeit von 211 Minuten am drittlängsten operiert. Dabei sollte Erwähnung finden, dass bei den beiden Patienten mit dem längsten (Patient 14, 240 Minuten) und dem drittlängsten (Patient 3, 211 Minuten) chirurgischen Eingriff zum Zeitpunkt der Operation eine morbide Adipositas bestand. Dabei wird deutlich, dass Individuelle Unterschiede bezüglich Gewicht, Allgemeinzustand und Anatomie der Patienten eine objektive Gegenüberstellung an dieser Stelle abschwächen.

Entgegengesetzt dazu zeigt sich ein Vergleich mit Operationszeiten der Arbeit von Sano et al. (2004). Die Arbeitsgruppe um Sano entfernte bei 263 Patienten im Rahmen der D2-LAD mit durchschnittlich 54 Lymphknoten (maximal 161, minimal 14) eine relativ höhere Anzahl an Lymphknoten, wobei sich mit 237 Minuten (maximal 625, minimal 127) im Vergleich ebenfalls höhere Werte für die durchschnittliche Schnitt-Naht-Zeit zeigen. Wie in unserem Kollektiv zeigt sich in der Studie von Sano et al. (2004) eine starke Variation der Operationsdauer. 


\section{Zusammenfassung}

Die Möglichkeit einer kurativen Therapie des lokal fortgeschrittenen Magenkarzinoms ist ausschließlich bei radikaler Gastrektomie unter Einbeziehung aller tumorinfiltrierten Lymphknoten gegeben.

In einer Kombination aus perioperativer Chemotherapie in Anlehnung an die MAGICStudie von Cunningham et al. (2006) und radikaler Chirurgie mit ausgedehnter Lymphadenektomie nach japanischem Vorbild kann somit ein vielversprechender Therapieansatz gesehen werden.

Ziel dieser Arbeit war es, bei Auswertung eines Kollektivs von 17 Patienten, bei denen zwischen November 2007 und Juli 2010 ein lokal fortgeschrittenes Magenkarzinom diagnostiziert wurde, den Behandlungserfolg der perioperativen Chemotherapie vor dem Hintergrund der Ergebnisse der MAGIC-Studie darzustellen und die chirurgische Radikalität der Gastrektomien innerhalb dieses Kollektivs unter Betrachtung internationaler Studienergebnisse zu beurteilen. Im Vergleich zum Primärstaging konnte bei 88,2\% (15 Fälle) der Patienten bei einem Restaging nach präoperativer Chemotherapie mittels ÖGD und EUS eine Remission des Primärtumors festgestellt werden. Bei 64,7\% (11 Fälle) der Patienten wurde zudem ein rückläufiger Größenbefund lokoregionärer Lymphknoten festgestellt. Der Anteil der am Operationsresektat pathologisch gesicherten Tumoren der UICC-Kategorien pT1 und pT2 betrug bei 16 Patienten mit totaler oder transhiatal erweiterter Gastrektomie 68,8\% (11 Fälle), davon war bei 31,3\% (5 Fälle) dieser Patienten der Primärtumor nach präoperativer Chemotherapie als Magenfrühkarzinom neu einzustufen. Zwischen dem letzten Zyklus der präoperativen Chemotherapie und der Operation vergingen innerhalb unseres Kollektivs durchschnittlich 30,75 Tage (minimal 6, maximal 57), zwischen Operation und erstem Zyklus der postoperativen Chemotherapie vergingen durchschnittlich 47,5 Tage (minimal 24, maximal 89).

18 Monate nach der Gastrektomie lebten noch 56,25\% der operierten Patienten, was sich durch Konformität zu den entsprechenden Überlebensraten der MAGIC-Studie (58\% CSC-Gruppe/ 54\% S-Gruppe) als positiv bewerten lässt. Es konnte kein Fall von direkter postoperativer Letalität festgestellt werden. 62,5\% (10 Fälle) der 16 Patienten nach Gastrektomie entwickelten bis zum Zeitpunkt der Auswertung ein Lokalrezidiv. 62,5\% (10 Fälle) der Patienten waren zum Zeitpunkt der Auswertung verstorben. 
In 93,8\% (15 Fälle) der Patienten, die einer Gastrektomie unterzogen wurden, wurde unabhängig vom UICC-Stadium mindestens eine D2-Lymphadenektomie (LAD) durchgeführt. Bei 87,5\% (14 Fälle) der Patienten handelte es sich um eine D2-LAD, während bei 6,3\% (1 Fall) Lymphknoten des dritten Kompartiments einbezogen wurden. Im Durchschnitt wurden 29,13 Lymphknoten in das Tumorresektat einbezogen (minimal 15, maximal 56).In durchschnittlich 4,56 Lymphknoten pro Patient waren vitale Tumorzellen nachzuweisen. Der Mittelwert für die Lymphknotenbefallsrate (lymph node ratio) betrug innerhalb der Patientengruppe $17,7 \%$ (maximal 82,6\%, minimal 0\%), wobei sich ein Zusammenhang zwischen längerem postoperativen Überleben und einer niedrigen „lymph node ratio“ andeutet. Dabei zeigt sich die lymph node ratio als entscheidender Prädiktor für das Outcome der Patienten, insbesondere für Patienten der N-Kategorien 1 und 2 (nach UICC).

Eine Gastrektomie dauerte innerhalb des Kollektivs durchschnittlich 180,75 Minuten (minimal 114, maximal 240).

In $37,5 \%$ aller endoskopischen Ultraschalluntersuchungen und in ebenfalls $37,5 \%$ aller CT-Untersuchungen im Rahmen der Ausbreitungsdiagnostik konnte das NStadium in Übereinstimmung zur histopathologischen Untersuchung des Resktats bestimmt werden.

Die Ergebnisse dieser Arbeit lassen den perioperativen Ansatz in der Therapie des lokal fortgeschrittenen Magenkarzinoms durchaus als vielversprechend bewerten, veranlassen aber $\mathrm{zu}$ einer zeitlichen Annäherung zwischen präoperativer Chemotherapie und Operation bei stabilem Allgemeinzustand des Patienten. Die gewonnenen Erkenntnisse stehen unter Einbeziehung frühzeitiger postoperativer Überlebensraten im Einklang mit der Studie von Cunningham et al. und deuten an, dass Patienten mit lokal fortgeschrittenem Magenkarzinom durch eine enge interdisziplinäre Therapieführung in Hinblick auf das Gesamtüberleben zu profitieren scheinen. Im Vergleich mit internationalen Studien zeigen sich die Ergebnisse zur Beurteilung der operativen Radikalität gut positioniert, bleiben aber hinter japanischen Werten für Operationsdauer, Anzahl entfernter Lymphknoten und Ausmaß der Lymphadenektomie zurück. Die von verschiedenen Autoren genannte prognostische Relevanz einer niedrigen Lymphknotenbefallsrate hinsichtlich des postoperativen Gesamtüberlebens lässt sich durch diese Arbeit untermauern 


\section{Literaturverzeichnis}

Ahn YO , Park BJ, Yoo KY Kim NK, Heo DS, Lee JK, Ahn HS, Kang DH, Kim H, Lee MS et al. (1991): Incidence estimation of stomach cancer among Koreans. J Korean Med Sci $\underline{6}, 7-14$

Bang Y, Chung H, Sawaki A, Xu J, Shen L, Lipatov O, Park SR, Gangadharan P, Advani SH, Kang YK (2008): HER2-positivity rates in advanced gastric cancer (GC): Results from a large international phase III trial. J Clin Oncol $\underline{26}$ (Abstract 4526)

Becker K, Mueller JD, Schulmacher C, Ott K (2003): Histomorphology and Grading of Regression in Gastric Carcinoma Treated with Neoadjuvant Chemotherapy.Cancer $\underline{98}$, $1521-1530$

Beer AJ, Wieder HA, Stollfuss JC (2007): Imaging staging of gastrointestinal tumors. Radiologie up2date $1,9-24$

Biffi R, Fazio N, Fabrizio L, Chiappa A, Andreoni B, Zampino MG, Roth A, Schuller JC, Fiori G, Orsi $F$ et al. (2010): Surgical outcome after docetaxel-based neoadjuvant chemotherapy in locally-advanced gastric cancer. World J Gastroenterol 16, 868-874

Bilici A, Seker M, Ustaalioglu BBO, Yilmaz B, Doventas A, Salepci T, Gumus M (2009): Determing of metastatic lymph node ratio in patients who underwent D2 dissection for gastric cancer. Med Oncol 27, 975-84

Böcker W, Denk H, Heitz PU, Moch H: Pathologie. 4.Auflage; Urban\&Fischer, München 2008

Boige V, Pignon JP, Saint-Aubert B, Lasser P, Conroy T, Bouché O, Segol P, Bedenne L, Rougier $P$, Ychou M (2007): Final results of a randomized trial comparing preoparativ 5 -fluorouracil $(\mathrm{F}) /$ cisplat in $(\mathrm{P})$ to surgery alone in adenocarcinoma of stomach and lower esophagus (ASLE). J Clin Oncol 로, 4510

Bonenkamp JJ, Hermans J, Sasako M, Van de Velde CJ (1999): Extended lymph node dissection for gastric cancer. N Engl J Med 340, 908-914

Borrmann R.: Geschwülste des Magens; in Handbuch der speziellen pathologischen Anatomie und Histologie IV/I . Springer Verlag, Berlin 1928

Bühling, KJ , Lepenies J , Witt K : Intensivkurs allgemeine und spezielle Pathologie. 3.Auflage , Urban\&Fischer, München 2004

Chen CH, Yang CC, Yeh YH (2002): Preoperative Staging of Gastric Cancer by Endoscopic Ultrasound: The Prognostic Usefulness of Ascites Detected by Endoscopic Ultrasound. J Clin Gastroenterol 35, 321-327 
Coburn NG, Swallow CJ, Kiss A, Law C (2006): Significant regional variation in adequacy of lymph node assessment and survival in gastric cancer. Cancer $\underline{107}$, 2143-2151

Crew KD, Neugut Al (2006) : Epidemiology of gastric cancer. World J Gastroenterol $\underline{12}, 354-362$

Cunningham D, Allum WH, Stenning SP, Thompson JN, Van de Velde CJH, Nicolson MD, Scarffe H, Lofts FJ, Falk SJ, Iveson TJ et al.: Perioperative chemotherapy versus surgery alone for respectable gastroesophageal cancer. N Eng J Med $\underline{355}, 11-20$

Curado MP, Edwards B, Shin HR, Storm H (2007): Cancer Incidence in Five Continents Vol.IX. 9. Auflage, International Agency for Research on Cancer (IARC), Lyon 2007

Cuscheri A, Weeden S, Fielding J, Bancewicz J, Craven J, Joypaul V, Sydes M, Fayers $P$ (1999): Patient Survival after D1 and D2 resections for gastric cancer: longterm results of the MRC randomized surgical trial. Surgical cooperative group. $\mathrm{Br} \mathrm{J}$ Cancer $\underline{79}, 1522-1530$

Desai AM, Pareek M, Nightingdale PG, Fielding JWL (2004): Improving outcomes in Gastric Cancer over 20 years. Gastric Cancer $\underline{7}, 196-201$

Dtsch. Gesellschaft für Chirurgie, Dtsch. Krebsgesellschaft (2003): Leitlinie Magenkarzinom. AWMF Online

Drzezga A, Stahl A, Wieder H, Meisetschläger G, Souvatzoglou M, Scheidhauer K, Schwaiger M (2005): Scintigraphic procedures in internal medicine. Dtsch Med Wochenschr 130, 2833-2842

Eisenhauer EA, Therasse P, Bogaerts J, Schwartz LH, Sargent D, Ford R, Dancey J, Arbuck S, Gwyther S, Mooney M et al. (2009): New response evaluation in solid tumors: Revised RECIST guideline (version 1.1). Eur J Cancer 4ㄷ, 228-247

Englisch-Fritz C, Hünerbein M, Porschen R (2008);: Diagnostik beim Magenkarzinom. Onkologe 14, 332-338

Feussner H: Lokale Behandlung von Magenfrühkarzinomen. In: Meyer HJ, Buhr HJ, Wilke H: Management des Oesophagus- und Magenkarzinoms. Springer Verlag, Berlin 2004, 259

Feussner H, Härtl F, Wilhelm D (2003): Extended Diagnostic Laparoscopy in the staging of gastrointestinal Tumors. Viszeralchirurgie $\underline{38}, 130-136$

Fischbach W (2004) : Gastrointestinal Lymphomas. Z Gastroenterol 42, 1067-1072

Fischbach W, Goebeler-Kolve ME, Dragosics B, Greiner A, Stolte M (2004) : Long term outcome of patients with gastric marginal zone B cell lymphoma of mucosa associated lymphoid tissue (MALT) following exclusive Helicobacter pylori eradication therapy: experience from a large prospective series. GUT $\underline{53}, 34-7$ 
Fuji M, Sasaki J, Nakajima T (1999): State of the art in the treatment of gastric cancer: From the $71^{\text {st }}$ Japanese Gastric Cancer Congress. Gastric Cancer 2, 151-157

Fujii M, Kochi M, Takayama T (2010): Recent Advances in Chemotherapy for Advanced Gastric Cancer in Japan. Surg Today $\underline{40}$, 295-300

Fukuda N, Sugiyama Y, Midorikawa A, Mushiake H (2009): Progonostic Significance of the Metastatic Lymph Node Ratio in Gastric Cancer Patients. World J Surg 33, 2378-2382

Gimelius B, Ekstrom K, Hoffmann K (1997): Randomized comparison between chemotherapy plus best supportive care with best supportive care in advanced gastric cancer. Ann Oncol $\underline{8}, 163-168$

Hamashima C, Shibuya D, Yamazaki H, Inoue K, Fukao A, Saito H, Sobue T (2008): The Japanese Guidelines for Gastric Cancer Screening. Jpn J Clin Oncol 38, 259-267

Hamers FPT, Gispen WH, Neijt JP (1999): Neurotoxic Side-effects of Cisplatin. Eur J Cancer $\underline{27}, 372-376$

Hartgrink HH, Van de Velde CJ, Putter H, Bonenkamp JJ, Klein Kranenbarg E, Songun I, Welvaart K, Van Krieken JH, Meijer S, Plukker JT et al. (2004): Extended lymph node dissection for gastric cancer: who may benefit? Final Results of the randomized dutch gastric cancer group trial. J Clin Oncol 22, 2069-2077

Hidaka H, Eto T, Maehara N, Jimi S, Hotokezaka M, Chijiiwa K (2008): Comperative effect of lymph nodes metastasis classified by the anatomical site or by the number of nodes involved on prognosis of patients with gastric cancer. Hepatogastroenterology $\underline{55}, 2269$

Hur H, Park CH (2009): Surgical Treatment of gastric carcinoma. Korean J Gastroenterol $\underline{54}, 83-98$

Hwang SW, Lee DH, Lee SH, Park YS, Hwang JH, Kim JW, Jung SH, Kim NY, Kim $\mathrm{YH}$, Lee KH et al. (2010): Preoperative Staging of Gastric Cancer by Endoscopic Ultrasonography and Multidetector-row Computed Tomography. J Gastroenterol Hepatol 25, 512-518

Hyoung Kim J, Song HY, Hoon Shin J, Hu HT, Lee SK, Jung HY,Yook JH (2009): Metallic Stent Placement in the Palliativ Treatment of Malignant Gastric Outlet Obstructions: Primary Gastric Carcinoma Versus Pancreatic Carcinoma. AJR 193, 241-247

Ichikura T, Tomimatsu S, Uefuji K, Tamakuma S (1999): Evaluation of The New American Joint Committee on Cancer/ International Union against Cancer classification of lymph node metastasis in gastric cancer defined by the fifth edition of the TNM classification in comparison with the Japanese classification. Cancer 86, 6859

Jaffe CC (2006): Measures of Response: RECIST, WHO and New Alternatives. J Clin Oncol 24, 3245-3251 
Jaffe ES, Harris NL, Stein H, Vardiman JW: World Health Organization and Classification of Tumours: Pathology and Genetics: Tumours of Haematopoetic and Lymphoid Tissues. Lyon; IARC-Press: Lyon 2001

Japanese Gastric Cancer Association (1998): Japanese Classification of Gastric Carcinoma $-2^{\text {nd }}$ English Edition. Gastric Cancer 1, 10-24

Jeong JY, Kim YJ, Han JK, Lee JM, Lee KH, Choi B, Yang HK, Lee KU (2004): Palliation of anastomotic obstructions in recurrent gastric carcinoma with the use of covered metallic stents: clinical results in 25 patients. Surgery $\underline{135}, 171-7$

Junginger $\mathrm{T}$, Hermanek $\mathrm{P}$, Klimpfinger $\mathrm{M}$ : Klassifikation maligner Tumoren des Gastrointestinaltraktes 2, 1.Auflage, Springer Verlag, Berlin 2002

Karamouzis MV, Konstantinopolus PA, Papavassiliou AG (2007): Trastuzumab: mechanism of action and use. N Engl J Med 357, 39-51

Kattan W, Karpeh S, Mazumdar M, Brennan F (2003) : Postoperative Nomogram for Disease-Specific Survival After an R0 Resection for Gastric Carcinoma. J Clin Oncol $\underline{21}, 3647-3650$

Kelley JR, Duggan JM (2003): Gastric cancer epidemiology and risk factors. J Clin Epidemiol $\underline{56}, 1-9$

Kollmannsberger C, Gerber E, Budach W, Borkermeyer C (2005): Rolle der systemischen \pm Radiotherapie beim resektablen Kardia-Magenkarzinom. Onkologe 11, 650-657

Kriplani AK, Kapur BM (1991): Laparoscopy for preoperative Staging and assessement of operability in gastric carcinoma. Gastrointest Endosc 37,441-443

Kris MG, Gralla RJ, Tyson LB, Clark RA, Kelsen DP, Reilly L, Groshen S, Bosl G, Kalman LA (2006): Improved control of cisplatin-induced emesis with high-dose metoclopramide and with combinations of metoclopramide, dexamethasone, and diphenhydramine. Results of consecutive trials in 255 patients. Cancer $\underline{55}, 527-534$

Kumar V, Abbas A, Fausto N, Mitchell R: Robbins Basic Pathology. 8.Auflage, Saunders ,Philadelphia 2007

Laurén P. (1965): The two histological main types of gastric carcinoma: diffuse and so called intestinal type carcinoma: an attempt at histo-clinical classification. Acta Pathol Microbiol Scand $\underline{64}, 31-43$

Lehnert T, Rudek B, Kienle P, Buhl K, Herfarth C (2002).: Impact of diagnostic laparoscopy on the management of gastric cancer: prospective study of 120 consecutive patients with primary gastric carcinoma. Br J Surg $\underline{89}, 471-475$

Louvet C, Andre T, Tigaud JM, Gamelin E, Douillard JY, Brunet R, Francois E, Jacob $\mathrm{JH}$, Taama A et al. (2002): Phase II study of oxaliplatin, fluorouracil, and folinic acid in locally advanced gastric or metastatic gastric cancer patients. J Clin Oncol 20, 45434548 
MacDonald JS, Smalley SR, Bendetti J, Hundahl SA, Estes NC, Stemmermann GN, Haller DG, Ajani JA, Gunderson LL, Jessup JM (2001): Chemoradiotherapy after surgery compared with surgery alone for adenocarcinoma oft he stomach or gastroesophageal junction. N Engl J Med $\underline{345}, 725-73$

Maehara Y, Hasuda S, Koga T, Tokunaga E, Kakeji Y, Sugimachi K (2000): Postoperative outcome and sites of recurrence in patients following curative resection for gastric cancer. Br J Surg $\underline{87}$, 353-357

Marelli D, Pedrazzani C, Roviello F (2009): Gastric Cancer Prognosis: Strong Correlation between Incidence and survival. Ann Surg Oncol 16, 2433-2441

Maruyama K, Gunven P, Okabayashi K, Sasako M, Kinoshita (1989): Lymph node metastases of gastric cancer. General pattern in 1931 patients. Ann Surg 210, 596602

McCracken M, Olsen M, Chen MS (2007): Cancer Incidence, mortality, and associated risk factors among Asian Americans of Chinese, Filipino, Vietnamese, Korean, and Japanese ethnicities. CA Cancer J Clin 57, 190-205

McMichael AJ, McCall MG , Hartshorne, JM , Wooding TL (1980) : Patterns of gastrointestinal cancer in European migrants to Australia: the role of dietary change. Int J Cancer 25 , 431-437

Menges M (2004): Diagnosis, Staging and Therapy of Gastric Cancer. Z Gastroenterol $\underline{42}, 767-773$

Messmann H, Schlottmann K (2001): Role of endoscopy in the staging of esophageal and gastric cancer. Semin Surg Oncol $\underline{20}$, 78-81

Meyer HJ, Wilke HJ (2011): Behandlungsstrategien beim Magenkarzinom. Dtsch Arzteblatt Int $\underline{108}, 698-706$

Minoru K, Yu T, Hiromi T, Keiji S, Yasuhis K, Tetsuya S, Hiroyuki O, Ryoichi M, Toshihiko S (2000): Treatment of Gastric Carcinoid. Stomach and Intestine 35, 14051415

Möhler M, Galle PR (2007) : Aktuelle Diagnostik und Therapie des Magenkarzinoms. Gastroenterologie up2date 3/2007, 17-25

Möning SP, Bollschweiler E, Hölscher AH (2005): Stellenwert der Lymphadenektomie im Behandlungskonzept des Magenkarzinoms. Viszeralchirurgie 40, 272-278

Möning SP, Baldus SE, Bollschweiler E, Hölscher AH (2007): Surgery of Gastric Cancer - Quality Management. Viszeralchirurgie 42, 82-88

Moretó M (2003): Diagnosis of Esophagogastric Tumors. Endoscopy $\underline{35}, 36-42$

Morgner A, Bayerdörfer E, Neubauer A, Stolte M (2001): Helicobacter pylori associated gastric B cell MALT lymphoma: predicitve factors for regression. GUT $\underline{48}$, 290-292 
Mortensen MB, Edwin B, Hühnerbein M, Liedman B, Nielsen HO, Hovendal C (2007): Impact of endoscopic ultrasonography (EUS) on surgical decision-making in upper gastrointestinal tract cancer: An international multicenter study. Surg Endosc 21, 431-8

O'Connel M (2004): PET-CT Modification of RECIST-Guidelines. J Natl Cancer Inst 96, 801-802

Öffner D, Meyer HJ: Chirurgische Onkologie. Springer Verlag, Wien 2008

Okuno K, Tanaka A, Shigeoka H, Hirai N, Kawai I, Kitano Y, Yasutomi M (1999): Suppression of T-cell function in gastric cancer patients after total gastrectomy with splenectomy: implications of splenic autotransplantations. Gastric Cancer $\underline{2}, 20-25$

Olayioye MA (2001): Update on HER-2 as a a target for cancer therapy: Intracellular signaling pathways of ErbB2/HER-2 and family members. Breast cancer Res $\underline{3}$, 385389

Parkin DM, Whelan SL, Ferlay J (1997): Cancer incidence in Five Continents. 2.Auflage, International Agency for Research on Cancer (IARC), Lyon 1997

Pfau PR, Chak A (2002): Endoscopic ultrasonography. Endoscopy 34 , 21-8

Pyrhonen S, Kuitunen T, Nyandoto P, Kouri M (1995): Randomised comparison of fluorouracil, epidoxorubicin and methotrexate (FEMTX) plus supportive with supportive care alone in patients with non-respectable gastric cancer. $\mathrm{Br} \mathrm{J}$ Cancer $\underline{71}$, 587-591

Ratain MJ, Eckhardt SG (2004): Phase II Study of Modern Drugs Directed Against New Targets: If You Are Fazed, Too, Then Resist RECIST. J Clin Oncol 22, 44424445

Riede UN , Werner M , Schäfer HE: Allgemeine und spezielle Pathologie. 5. Auflage; Thieme Verlag, Stuttgart 2004

Robert-Koch-Institut, Gesellschaft d. epidemiologischen Krebsregister in Deutschland e.V. : Krebs in Deutschland 2003-2004. Häufigkeiten und Trends. 6.Auflage, RobertKoch-Institut ,Berlin 2008

Ross $\mathrm{P}$, Nicolson M, Cunningham D, Valle J, Seymour M, Harper P, Price T, Anderson $\mathrm{H}$, Iveson $\mathrm{T}$, Hickish $\mathrm{T}$ et al. (2002): Prospective randomized trial comparing mitomycin, cisplatin, and protected venous-infusion fluorouracil (PVI 5-FU) with epirubicin, cisplatin and PVI 5-FU in advanced esophagogastric cancer. J Clin Oncol 20, $1996-2004$

Roukos DH (2000): Current status and future perspectives of gastric cancer management. Cancer Treat Rev $\underline{26}, 243-55$

Sano T, Katai H, Sasako M, Maruyama K (2002): One thousand consecutive gastrectomies without operative mortality. Br J Surg $\underline{89}, 123$

Sano T, Sasako M, Yamamoto M, Nashimoto A, Kurita A, Hiratsuka M, Tsujinaka T, Kinoshita T,Arai K,Yamamura Y,Okajima K (2004): Gastric Cancer Surgery: Morbidity and Mortality Results From a Prospective Randomized Controlled Trial Comparing D2 
and Extended Paraaortic Lymphadenectomy - Japan Clinical Oncology Group Study 9501. J Clin Oncol 22, 2767-2773

Sasako M (1997): Risk factors for surgical treatment in the Dutch gastric cancer trial. Br J Surg 84, 1567-1571

Sasako M, Sano T, Yamamoto S, Kurokawa Y, Nashimoto A, Kurita A, Hiratsuka M, Tsujinaka T,Kinoshita T, Arai K et al. (2008): D2 Lymphadenectomy Alone or with Paraaortic Nodal Dissection for gastric cancer. N Engl J Med 359, 453-462

Schmidt-Matthiesen A, Bechstein WO (2005):Magenkarzinom. Viszeralchirurgie $\underline{40}$, 68-74

Schröder W, Möning SP, Hölscher AH (2001): Magenfrühkarzinom. Endoskopische Ablatation oder onkologische Resektion aus Sicht eines Viszeralchirurgen. Chir Praxis $\underline{59}, 29-44$

Schrübel S, Faiss S, Zeitz M (2003): Neuroendocrine gastrointestinal Tumorsdiagnosis and therapy. Dtsch Med Wochenschr 128, 81-83

Seidman A, Hudis C, Pierri MK, Shak S, Paton V, Ashby M, Murphy M,Stewart SJ, Keefe D (2002) : Cardiac dysfunction in the trastuzumab clinical trials experience. J Clin Oncol 20, 215-21

Sendler A, Siewert JR (2000) : Diagnostik und Staging beim Magenkarzinom. Dtsch Med Wochenschr 125, 323-326

Sendler A, Böttcher K, Etter M, Siewert JR (2000): Das Magenkarzinom. Internist $\underline{41}$, 817-830

Sendler A, Etter M, Böttcher K, Siewert JR (2002): Resektionsausmaß in der Chirurgie des Magenkarzinoms. Chirurg $\underline{73}, 316-324$

Shchepotin I, Evans SR, Shabahang M, Cherny V,Buras RR, Zadorozhny A, Nauta RJ(1995): Radical treatment of locally recurrent gastric cancer. Am Surg 61,371-376

Siewert JR (2004): Auf der Suche nach der individuellen Indikation zur D2Lymphadenektomie beim Magenkarzinom. Chirurg $\underline{75}, 1145-1150$

Siewert JR (2006): Magenkarzinom: Ist die OP mit perioperativer Chemotherapie der alleinigen OP überlegen? Studienergebnisse sprechen dafür. Gastroenterologie up2date $\underline{2}, 260-261$

Siewert JR, Brauer RB: Basiswissen Chirurgie. Springer Verlag, Wien 2009

Siewert JR, Kestlmeier R, Busch R, Böttcher K, Roder JD, Müller J, Fellbaum C, Höfler H (1996): Benefits of D2 lymph node dissection for patients with gastric cancer and pN0 and pN1 lymph node metastases. Br J Surg $\underline{83}$, 1144-1147

Siewert JR, Böttcher K, Stein H, Roder JD (1998): Relevant prognostic factors in gastric cancer. Ann Surg 228, 449-461 
Siewert JR, Stein HJ, Feith M : Adenokarzinom des gastroösophagealen Übergangs (AEG-Karzinom), sog. Kardiakarzinom. In: Praxis der Viszeralchirurgie. 2.Auflage, Springer Verlag, Berlin 2006

Therasse P, Arbuck SG, Eisenhauer EA, Wanders J, Kaplan RS, Rubinstein L, Verweij J, Van Glabbeke M, Van Oosterom AT, Christian MC, Gwyther SG (2000): New Guidelines to Evaluate the Response to Treatment in Solid Tumors. J Natl Cancer Inst $\underline{92}, 205-216$

Thomas C : Histopathologie. 14.Auflage, Schattauer Verlag, Stuttgart , 2006

Tsubono Y, Hisamichi S (2008): Screening for gastric cancer in Japan. Gastric Cancer $\underline{3}, 9-18$

UICC (Union internationale contre le cancer), Klassifikation in: Wittekind C, Meyer H-J, Bootz F: UICC: TNM Klassifikation maligner Tumoren. 6. Auflage. Springer, Berlin 2002

Untch M, Jackisch C, Thomssen C, Nitz U, von Minckwitz G, Kaufmann M (2006): Adjuvante Therapie mit Trastuzumab bei Mammakarzinompatientinnen. Dtsch Arztebl 103, A-3406/ B-2961/ C-2841

Van Cutsem E, Kang Y, Chung H, Sawaki A, Lordick F, Hill J, Lehle M, Feyereislova A, Bang Y (2009): Efficacy results from the ToGA trial: A Phase III study of trastuzumab added to standard chemotherapy in first-line human epidermal growth factor 2 (HER2)-positive advanced gastric cancer. J Clin Oncol 27, 18

Van de Velde CJ (2005): Resection for gastric cancer in the community. Semin Oncol $\underline{32}, 90-3$

Vanhoefner U, Schleuchner N (2004): Magenkarzinom - adjuvante und palliative Chemotherapie. Onkologe $\underline{10}, 11-13$

Vieth M (2008): Entzündliche Magenerkrankungen und Helicobacter pylori: state of the art - Risikofaktoren für ein Magenkarzinom. Klinikarzt 37, 344-348

Virányi Z, Velósy B, Madácsy L (2006): Early gastric cancer: From recognition to solution.Z Gastroenterol $\underline{44}, 719-726$

Wanebo HJ, Kennedy BJ, Chmiel J, Steele G, Winchester D, Osteen R (1993): Cancer of the stomach: a patient care study by the American college of surgeons. Ann Surg $\underline{218}, 583-592$

Wang Z, Chen JQ, Cao Y-F (2010): Systematic review of D2 lymphadenectomy versus D2 with para-aortic nodal dissection for advanced gastric cancer. World $\mathrm{J}$ Gastroenterol 16: 1138-1149

Webb A, Cunningham D, Scarffe JH, Harper P, Norman A, Joffe JK, Hughes M, Mansi J, Findlay M, Hill A et al (1997): Randomized trial comparing epirubicin, cisplatin, and fluorouracil versus fluorouracil, doxorubicin, and methotrexate in advanced esophagogastric cancer. J Clin Oncol 15, 261-7 
Wilke H, Stahl M, Meyer H-J, Arnold D: Kompendium internistische Onkologie. Springer Verlag, Wien 2006

Willis S, Troung S, Gribnitz S, Fass J, Schumpelick V (2000).: Endoscopic ultrasonography in the preoperative staging of gastric cancer. Surg Endosc 14, 951954

Wittekind C, Meyer H-J: TNM Klassifikation maligner Tumoren. 7.Auflage. VCH-Wiley, Weinheim 2010

Wittekind C, Meyer H-J, Bootz F: TNM Klassifikation maligner Tumoren. 6.Auflage. Springer, Berlin 2002

Wittekind C, Klimpfinger M, Sobin LH: UICC: TNM Atlas. Illustrierter Leitfaden zur TNM/pTNM-Klassifikation maligner Tumoren. 5. Auflage. Springer, Berlin 2005

Wu CW, Chang IS, Lo SS, Hsieh MC, Shia LT, Wang-Peng J (2006): Complications following D3 gastrectomy: post hoc analysis of a randomized trial. World J Surg $\underline{30}$, 12-16

Xu D, Geng Q, Long Z, Zhan Y, Li W, Zhou Z, Chen Y, Sun X, Chen G, Liu Q (2009): Positive Lymph node Ratio Is an Independent Prognostic Factor in Gastric Cancer After D2 Resection Regardless of the Examined Number of Lymph nodes. Ann Surg Oncol 16, 319-326

Yamamoto S (2001) : Stomach cancer incidence in the world. Jpn J Clin Oncol 31, 471

Yamashita K, Sakuramoto S, Kikushi S, Katada N, Kobayashi N, Watanabe M (2008): Validation of staging systems for gastric cancer. Gastric Cancer 11, 111-118

Yasuda K (2006) : Early gastric cancer: diagnosis, treatment techniques and outcomes. Eur J Gastroenterol Hepatol 18, 839-845

Zhao SL, Fang JY (2008): The role of postoperative adjuvant chemotherapy following curative resection for gastric cancer: a meta-analysis. Cancer Invest $\underline{26}$, 317-325

Zittel TT (2002): Therapy of gastric cancer. Viszeralchirurgie 37, 262-268 


\section{Danksagung}

Ich bedanke mich bei Professor Dr. med. Dr. h.c. Giuliano Ramadori, Leiter der Abteilung Gastroenterologie und Endokrinologie des Zentrums Innere Medizin der Universitätsmedizin Göttingen, für die freundliche Überlassung des Themas und für zahlreiche anregende Unterhaltungen während der Entstehung dieser Arbeit.

Mein besonderer Dank gilt Gwen-Jana Töppler, Assistenzärztin in der Abteilung Gastroenterologie und Endokrinologie des Zentrums Innere Medizin der Universitätsmedizin Göttingen, für ihr stets offenes Ohr bei klinischen Fragen und ihre motivierenden Worte in Zeiten erhöhter Belastung.

Für die Hilfe bei Darstellung und statistischer Auswertung meiner Ergebnisse gilt mein Dank den Mitarbeitern der Abteilung Medizinische Statistik der Universitätsmedizin Göttingen, insbesondere Hans-Joachim Helms und Simon Schneider.

Frau Sandkuhl, Sekretariat Prof. Dr. med. G. Ramadori, danke ich für ihre Geduld und Hilfsbereitschaft. 